\author{
Diakonie-Krankenhaus Harz GmbH \\ Brockenstraße 1 \\ 38875 Elbingerode \\ Ärztlicher Direktor: Dr. med. S. Gerhardt \\ Geschäftsführer: Sr. Anita Rost \& \\ Martin Montowski
}

\title{
Zugangsvariablen als Prädiktoren für das Therapieergebnis bei Alkohollangzeitentwöhnungsmaßnahmen
}

\author{
Dissertation zur Erlangung des Doktorgrades der Medizin \\ der Medizinischen Fakultät der \\ Universität UIm
}

von

Eckart Grau

geb. in Stuttgart

Juli 2011 
Amtierender Dekan:

Prof. Dr. Thomas Wirth

1. Berichterstatter:

Prof. Dr. med. Peter Brieger

2. Berichterstatter

Prof. Dr. med. Markus Jäger

Tag der Promotion

18.10.2012 


\section{Inhaltsverzeichnis}

Inhaltsverzeichnis.

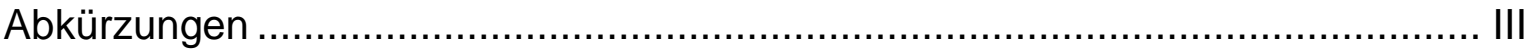

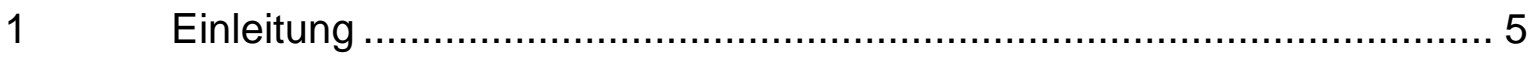

$1.1 \quad$ Fragestellungen und Hypothesenbildung ....................................... 8

$2 \quad$ Material und Methoden ………………........................................ 12

2.1 Themenbetrachtung, Hintergrund ............................................... 12

2.2 Zugangssteuerung zur Medizinischen Rehabilitation .......................... 16

2.3 Forschungsüberblick ............................................................... 16

2.3.1 Bisherige Entwicklungen ........................................................... 16

2.3.2 Die Rehabilitanden-Management-Kategorien (RMK) ........................ 17

2.3.3 Überblick über Studienergebnisse der Rehabilitationsforschung ........... 24

2.3.4 Schlussfolgerungen aus den Studienergebnissen .............................. 32

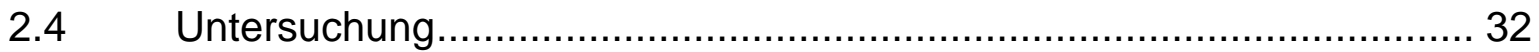

2.4.1 Ort und strukturelle Umgebung der Untersuchung.............................. 32

2.4.2 Überblick über die Methodik der Untersuchung …................................ 33

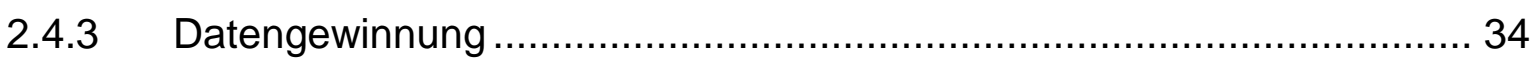

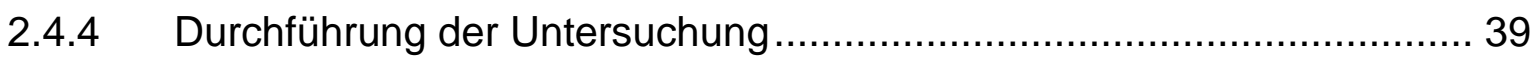

2.4.5 Darstellung der Untersuchungsstichprobe ....................................... 42

2.4.6 Darstellung der Outcome-Variablen ................................................ 47

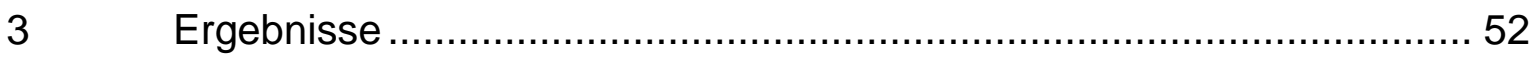

3.1 Darstellung der Ergebnisse ........................................................ 52

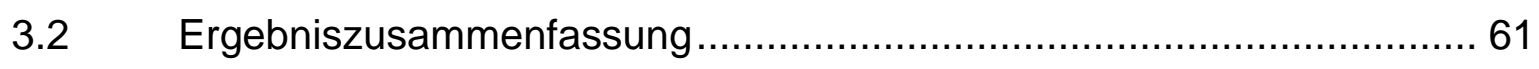

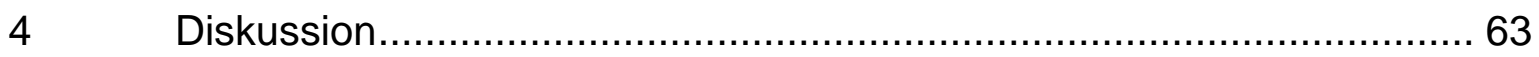


4.1 Fazit für die Praxis

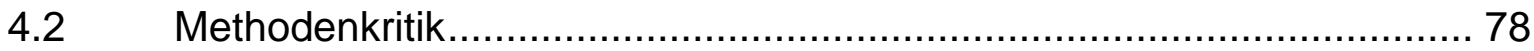

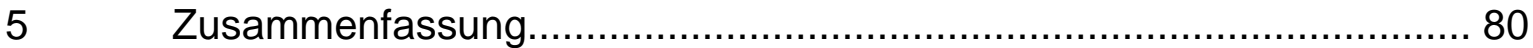

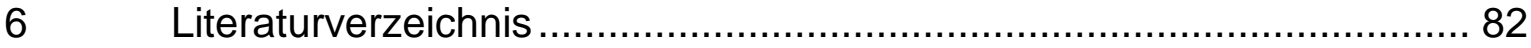

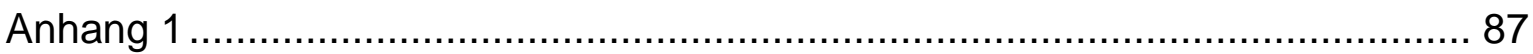

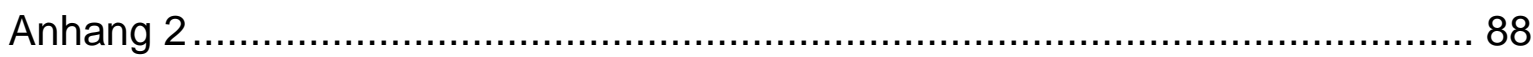

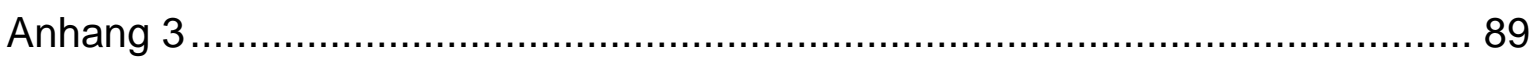

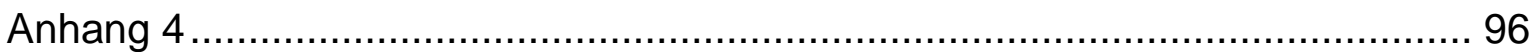

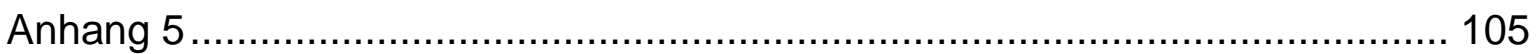

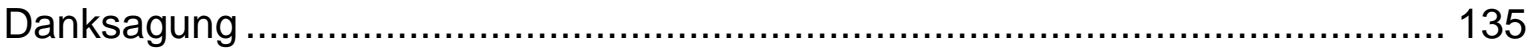

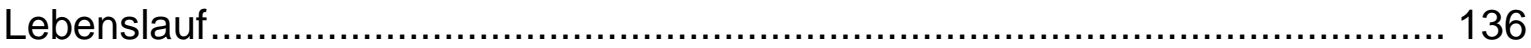




\section{Abkürzungen}

AHG

AL-1, AL-2, AL-3, AL-4

Allgemeine Hospitalgesellschaft AG mit Sitz in Düsseldorf

AWMF

Bado

BfA

c.l.-Skala

df

DGD

DGSS

$\mathrm{DKH}$

DSM-IV

DRG

ETM

$\operatorname{Exp}(B)$

FVS

hyp

ICD

ICF

KTL-System

lin

$\log$

Log-Likelihood die resultierenden

Bedarfsgruppen

für

alkoholabhängige Patienten

Arbeitsgemeinschaft der wissenschaftlichen medizinischen Fachgesellschaft

Basisdokumentation

Bundesversicherungsanstalt für Angestellte (alte Bezeichnung der heutigen Deutschen

Rentenversicherung Bund)

Selbstbeurteilungsverfahren zur Objektivierung leichter cerebraler Insuffizienzen

„degrees of freedom“; steht in der Statistik für Freiheitsgrade

Deutscher Gemeinschafts-Diakonieverband e. V.

Deutsche Gesellschaft für Suchtforschung und Suchttherapie e.V.

Diakonie-Krankenhaus Harz GmbH

Diagnostisches und statistisches Manual psychischer Störungen

Diagnosebezogene Fallgruppen

evidenzbasierte Therapiemodule

Effektkoeffizient bei der logistischen Regression

Fachverbund Sucht e. V.

Hypothese

Internationale Klassifikation der Krankheiten

Internationale Klassifikation der Funktionsfähigkeit,

Behinderung und Gesundheit

Katalog Therapeutischer Leistungen

lineare Regression

logistische Regression

logarithmierte Likelihood-Funktion 
LVA

MS Excel

POS

PSSI

$R^{2}$

RK

RMK

RTS

SCL-90-R

SPSS

VEG

Wald

WST
Landesversicherungsanstalt (alte Bezeichnung für regionalen Deutschen Rentenversicherungen)

Microsoft Excel

Polytechnische Oberschule

Persönlichkeits-Stil-und-Störungs-Inventar

Bestimmtheitsmaß; ein statistisches Maß für den erklärten Anteil der Varianz einer abhängigen Variablen $Y$ durch ein statistisches Modell

Referenzkategorie

Rehabilitanden-Management-Kategorien

Rehabilitationstherapiestandards

Symptomcheckliste (mit 90 Items, in revidierter Fassung)

"Statistical package for the social sciences" (StatistikProgramm)

Verbalgedächtnis Test

Bezeichnung für einen statistischen Hypothesentest Wortschatztest 


\section{Einleitung}

Im Rahmen der klinischen Erfahrung aus mehreren Jahren medizinischtherapeutischer Arbeit mit alkoholabhängigen Patienten sowie im Erfahrungsaustausch mit Kollegen, die in dem selben Fachgebiet tätig sind, wurde deutlich, dass sowohl Prädiktoren für eine erfolgreiche Entwöhnungstherapie, als auch Wirkfaktoren innerhalb einer Entwöhnungstherapie, sehr schwer zu fassen und zu definieren sind. Die Prädiktion (lat. praedicere - voraussagen) ist eine Aussage über Ereignisse, Zustände oder Entwicklungen in der Zukunft, in diesem Fall der Verlaufsentwicklung bzw. dem Erfolg einer Alkohollangzeitentwöhnungstherapie. Dass diese prognostische Beurteilung bei Suchterkrankungen überdurchschnittlich schwierig ist, dürfte in weiten Stücken an dem Krankheitsbild selbst liegen. Es finden sich schließlich viele irrationale und paradoxe Elemente im Bereich Entstehung, Erhaltung und Behandlung von Suchterkrankungen. So ist das Verhalten von Suchtkranken ausgesprochen schwierig vorauszusehen. Abläufe sind oft nicht planbar, Strategien zur Abstinenzsicherung greifen in der praktischen Umsetzung häufig nicht mehr. Die Antizipationsfähigkeit des Suchtkranken und seine Möglichkeiten, glaubhaft vorgenommene Strategien umzusetzen, sind in Situationen der Belastung und der Versuchung oft defizitär. Es kommt zu Verhaltensrückfällen und stofflichen Rückfällen, da der Betroffene sich plötzlich Kräften ausgesetzt sieht, die ihn zum Konsum führen wollen. Trotz teilweise langjähriger Erfahrung mit dem Suchtmittel erlebt er diese verführende Konfrontation immer wieder unerwartet und fühlt sich ungeachtet ehrlicher, willentlicher Abstinenzmotivation hilflos und handlungsunfähig. „Sucht ist ein unabweisbares Verlangen nach einem bestimmten Erlebniszustand. Diesem Verlangen werden die Kräfte des Verstandes untergeordnet. Es beeinträchtigt die freie Entfaltung einer Persönlichkeit und zerstört die sozialen Bindungen und die sozialen Chancen des Individuums." (Wanke 1985, Seite 20) Ein weiterer, krankheitsimmanenter Aspekt ist die Paradoxie des Symptoms. Im Regelfall erleben wir Krankheitssymptome wie Schmerz, Unwohlsein, Depressivität, Schlafstörung etc. als unangenehm und lebensfeindlich. Der Wunsch der Symptombeseitigung treibt den Betroffenen in Richtung helfendes System, wie Krankenlager, Arzt, Medikamente, Krankenhaus, etc. Bei der Krankheitsentwicklung einer Sucht wird das Symptom, welches später die Abhängigkeit bedingt, nämlich der rauschhafte Konsum des Suchtmittels, 
dagegen über einen langen Zeitraum positiv erlebt und deshalb laufend gezielt gesucht. Es wird wiederholt herbeigeführt und gegen die Menschen, die eine Konsumreduktion empfehlen, verteidigt. Der Suchtkranke erlebt also den Konsum des Mittels, welches auf seine seelische, organische und soziale Zerstörung ausgerichtet ist, als positive, ich-syntone Handlung. Aus der Erfahrung ist bekannt, dass genau dieses Phänomen die Behandlungsschwierigkeit von Suchtkranken bedingt. Für die hier getätigten Betrachtungen ist dieser Umstand aber aus einem anderen Grund entscheidend. Für das suchtbehandelnde System kann wegen des beschriebenen Phänomens die strukturelle Komponente der Patientenversorgung, d. h. die Planbarkeit von Behandlungsabläufen, die Beschreibung von Prädiktoren und Wirksamkeitsfaktoren sowie die Formulierung von Markern für prognostische Zusatzaussagen, enorm erschwert werden. Es liegen unter anderem auch deshalb keine klar messbaren Faktoren vor, die eine gesicherte Klassifizierung von Suchtkranken bzw. ihrer Krankheitsintensität ermöglichen. Somit gibt es also keinen allgemein gültigen Katalog, welcher diesen Patienten hinsichtlich ihres Versorgungsbedarfs gerecht wird. Auch sind therapierelevante Faktoren, wie Vertrauensfähigkeit von Patienten, aber auch Fähigkeiten wie Vertrauensförderung durch Therapeuten und Ärzte, ausgesprochen schwer zu fassen. Hier zeigen sich durchaus auch Grenzen der testpsychologischen Kompetenz. Dies bedeutet Unklarheiten und Unschärfen hinsichtlich Behandlungsintensität, Betreuungsaufwand, Dauer der Begleitung, Notwendigkeit der Belastungsschonung, Art und Ausmaß der medikamentösen Betreuung und Akzeptanz bzw. Integration von chronischen Defiziten. Alle beschriebenen Faktoren erschweren zudem eine angemessene sozialmedizinische Beurteilung.

Aus der praktischen Erfahrung ist dem Autor und seinem Behandlungsteam für Abhängigkeitserkrankungen als sicherer Prädiktor lediglich der vom Patienten empfundene Leidensdruck bewusst. Aufgrund der Tatsache, dass ein Mensch nur dann eine tiefgreifende Veränderung durchführen will und wird, wenn er die gegenwärtigen Umstände seines Daseins nur noch sehr schlecht bzw. nicht mehr aushält, wird auch in der Behandlung von Suchterkrankungen nur dann eine konstruktive Veränderungsarbeit möglich sein, wenn die negativen Elemente und Konsequenzen des Suchtmittelkonsums gegenüber den positiven überwiegen. Erfahrungsgemäß kann es sehr lange dauern, bis dieser Zustand erreicht wird und meist sind die Beobachter und Begleiter (im Regelfall die nächsten Angehörigen) 
sehr viel schneller im Bereich eines Leidensdruckes angelangt, der Handlungsbedarf erweckt, als der Betroffene selbst. Da die Abwehrmechanismen des Suchtkranken vielfältig und massiv sind, sind häufig nicht nur die rationalen Steuermöglichkeiten des Betroffenen selbst überfordert. Auch die Möglichkeiten der Behandelnden, die vor allem an vernunftorientierte Verhaltensweisen appellieren, die das Bedenken von Konsequenzen oder Überlegungen im Sinne einer Güterabwägung vermitteln wollen, werden rasch erschöpft und sind sehr begrenzt in der Lage, Veränderung zu induzieren. Insbesondere ist an dieser Stelle die Verleugnung als einer der Hauptabwehrmechanismen von Suchtkranken zu nennen. Dieser ermöglicht es dem Betroffenen, Wirklichkeiten auszublenden und Konsumverhalten zu rechtfertigen sowie eigene Möglichkeiten in gravierender Weise falsch einzuschätzen, ohne dabei Irritationen und Widersprüche selbst wahrzunehmen. Die Beschreibung von Prädiktoren und Wirkfaktoren ist deshalb zusätzlich erschwert, da die anamnestischen Angaben und Schilderungen eines Suchtkranken im Falle einer ausgeprägten, verleugnenden Abwehr nicht der Realität entsprechen. Es wird an dieser Stelle ersichtlich, wie schwer es ist, objektive Parameter für die gewünschte Klassifizierungsgrundlage zu erhalten.

Ausgehend von der Tatsache, dass sich Suchtkrankheiten, insbesondere Alkoholabhängigkeit, durch nahezu alle Kulturkreise, Gesellschaftsschichten und Altersgruppen ziehen, stellte sich die Frage, ob es überhaupt möglich sein kann, einzelne Zugangsvariablen für eine Langzeitentwöhnungsmaßnahme zu definieren, d. h. aus bekannten Variablen zu isolieren, die im Sinne einer gewünschten Eindeutigkeit und womöglich quantitativer Messbarkeit prädiktorisch eine Beurteilungssicherheit für den ärztlich-therapeutischen Behandler erbringen können. Hinter dem so unscharf umrissenen Begriff des Alkoholkranken verbergen sich schließlich verschiedenste Persönlichkeiten, Sozialisationen, Möglichkeiten und Grenzen von betroffenen Menschen, also im Prinzip eine Vielzahl von Krankheitsbildern.

Ein besonderes Augenmerk sollte in dieser Untersuchung zunächst auf den Zugangsweg des Betroffenen zur Rehabilitationseinrichtung gerichtet sein. Ziel war zu überprüfen, ob dieser eine Bedeutung hat, was den Gesamtablauf der Genesung eines Alkoholkranken angeht. Bei beschriebener, geringer Eindeutigkeit hinsichtlich prädiktorisch nützlicher Faktoren, gilt für den Zugangsweg immerhin, dass jeder Patient einen solchen benennen kann und dass eine hohe 
Vergleichbarkeit gegeben ist. Die Betrachtung der Tatsache, ob ein Patient beispielsweise über seinen Hausarzt, die Suchtberatungsstelle, eine Direktverlegung aus der entgiftenden Klinik, durch Gericht oder Arbeitgeber mit Auflage etc. die Langzeitentwöhnungsmaßnahme beginnt, erschien also aus zwei Gründen als sinnvoll. Zum Einen sind strukturelle Abläufe, wie Zugangswege, recht eindeutig definiert und zurück zu verfolgen. Zum Anderen wäre es in dem Fall, dass sich ein Zugangsweg als günstiger hinsichtlich des Therapieoutcomes erweisen würde als ein anderer, recht einfach, eine verbessernde Korrektur im Bereich Zugangssteuerung durchzuführen.

In der vorliegenden Arbeit wird der Patientenjahrgang 2004 des DiakonieKrankenhauses Harz GmbH in Elbingerode (DKH) untersucht. Das Interesse richtet sich auf die Patienten mit der Haupt- und damit Einweisungsdiagnose Alkoholabhängigkeit. Ziel war hierbei das Auffinden von messbaren und für die therapeutische Arbeit relevanten Zugangsvariablen, die im allgemeingültigen Sinn prädiktorisch sind und für die Behandlung von alkoholabhängigen Menschen nutzbar gemacht werden können.

\subsection{Fragestellungen und Hypothesenbildung}

\section{Fragen}

- Existieren abgrenzbare Prädiktoren für einen Rehabilitationserfolg bei der Behandlung von Alkoholabhängigen, wenn man die Zugangswege zur Rehabilitation und das entsprechende Rehabilitationsergebnis miteinander vergleicht?

- Können unter anderen Zugangsvariablen für die Rehabilitation von Alkoholabhängigen einzelne Merkmale bzw. Gruppen von Merkmalen gefunden werden, die prädiktorisch für ein günstiges oder ungünstiges Rehabilitationsergebnis zu sehen sind?

- Ist das Problem der hohen Nichtantrittsrate von Alkoholabhängigen trotz kostengewährter Therapiemaßnahme ggf. ein Forschungsfokus höherer 
Dringlichkeit, als der Versuch einer weiteren Optimierung der Zugangssteuerung?

- Kann die vorliegende Arbeit einen impulsgebenden Beitrag zur aktuellen Rehabilitationsforschung mit ihrem gegenwärtigen Forschungsansatz, den Fallgruppen, leisten?

- Welche Bedeutung am Therapieergebnis kommt der Therapiemotivation des Patienten zu?

- Welche Bedeutung hat die Art der motivationalen Abweichung von der Eigenmotivation, d. h. welche Bedeutung hat die Tatsache, ob eine Fremdmotivation oder eine ambivalente Therapiemotivation vorliegt?

- Finden sich prädiktorisch aussagekräftige Variablen im sozialen oder beruflichen Bereich, die über die Frage der unmittelbaren Abstinenzsicherung hinaus hinsichtlich des Gesamttherapieergebnisses relevant sein könnten?

\section{Hypothesen}

Unter Berücksichtigung der formulierten Frageninhalte wurden Hypothesen gebildet. Hierbei war für die erste Haupthypothese der motivationale Aspekt im Vordergrund zu sehen. Zum einen ist aus der praktischen Erfahrung bekannt, dass die Motivation eine wichtige Größe für das therapeutische Ergebnis darstellt, zum anderen findet sich gerade im Bereich der Zugangswege eine Besonderheit, die unmittelbar mit der Therapiemotivation eines Patienten zusammenhängt. So gibt es bei der Betrachtung der unterschiedlichen Zugangswege mehrere „beauflagte“ Wege, sei es durch das Gericht, den Arbeitgeber oder die Agentur für Arbeit. Bei dieser Gruppe ist der Anteil der fremdmotivierten Patienten eindeutig erhöht, alle anderen in dieser Arbeit überprüften Zugangsvariablen sind aber unbeeinflusst verteilt.

Die zweite Haupthypothese bezieht sich auf die prädiktorische Aussagekraft aller weiteren untersuchten Zugangsvariablen. Die dritte Haupthypothese bezieht sich auf den Vergleich zwischen bereits vorliegenden Ergebnissen der 
Rehabilitationszugangssteuerung und den in dieser Arbeit gewonnenen Ergebnissen.

Im Einzelnen wurden also folgende Haupthypothesen überprüft:

1) Es ist davon auszugehen, dass Patienten, die zu Beginn der Langzeittherapie explizit zum Ausdruck bringen, unfreiwillig bzw. fremdmotiviert gekommen zu sein (weil sie beispielsweise über die Arbeitsagentur, das Gericht oder durch den Arbeitgeber beauflagt wurden), ein schlechteres Outcome haben als Patienten mit hoher Eigenmotivation. Auch ist zu erwarten, dass die Patienten, die selbst eine noch ambivalente Therapiemotivation zum Ausdruck bringen, ein schlechteres Outcome haben als Patienten, denen eine Eigenmotivation attestiert werden kann (deduktive Hypothese).

2) Weitere Aspekte des Zugangswegs und zu Therapiebeginn vorliegende zugangsrelevante Patientenmerkmale sind Prädiktoren für das Therapieergebnis (deduktive Hypothese).

3) Es ergeben sich aus den patientenbezogenen Daten (siehe Haupthypothese 1 und 2) Merkmalsbereiche, die die zugangsrelevanten Aspekte wesentlich abbilden. Es ist dabei davon auszugehen, dass diese Merkmalsbereiche weitgehend den therapierelevanten Subdimensionen aus der RehabilitandenManagement-Kategorien (RMK) Forschung Sucht (Egner et al. 2009) zuzuordnen sind (siehe 2.3.2). Im Einzelnen wären dies: die „substanzenbezogene Beeinträchtigung“, die „psychische Symptomatik“, die „persönlichen Ressourcen“, das „arbeitsbezogene Erleben und Verhalten“, „das Alter und die Arbeitslosigkeit“ sowie die „soziale Unterstützung“ (induktive Hypothese).

Die spezifischen Hypothesen differenzieren die in den Haupthypothesen dargestellten Inhalte. In der spezifischen Hypothese 1 werden die unterschiedlichen Zugangsmerkmale, die die Motivationslage betreffen, auf Zusammenhänge mit den Outcomevariablen (siehe Punkt 2.4.6) überprüft, in der spezifischen Hypothese 2 geschieht dies im Hinblick auf alle weiteren Zugangsvariablen. Eine spezifische Hypothese 3 wurde nicht formuliert, da die Haupthypothese 3 einem Ergebnisvergleich entspricht. 
Spezifische Hypothese 1

1 a Bei Patienten "mit Auflage" sowie bei Fremdmotivation bzw. ambivalenter Motivation findet sich eine höhere Abbruchrate, d. h. die Therapie wird signifikant seltener „regulär“ bzw. „vorzeitig mit ärztlich-therapeutischem Einverständnis" beendet.

$1 \mathrm{~b}$ Bei Patienten "mit Auflage“ sowie bei Patienten mit ambivalenter Therapiemotivation bzw. Fremdmotivation ergibt sich ein von den Therapeuten schlechter eingeschätztes Therapieergebnis als bei den Patienten mit Eigenmotivation.

1c Es findet sich bei dieser Patientengruppe ein niedrigerer Anteil an vollschichtiger Leistungsfähigkeit „bezogen auf den allgemeinen Arbeitsmarkt" als bei Patienten "ohne Auflage“ bzw. bei eigenmotivierten Patienten.

$1 \mathrm{~d}$ Es wird eine niedrigere Abstinenzrate zum Katamnesezeitpunkt ausgewiesen.

$1 \mathrm{e}$ Ein Jahr nach Beendigung der Therapie liegt ein geringerer Anteil an Erwerbstätigkeit vor.

Spezifische Hypothese 2

2 a Es bestehen Zusammenhänge zwischen den definieren Zugangsvariablen und der Entlassform.

$2 \mathrm{~b}$ Es bestehen Zusammenhänge zwischen den Zugangsvariablen und dem vom Therapeuten eingeschätzten Therapieergebnis.

$2 \mathrm{c}$ Es bestehen Zusammenhänge zwischen den Zugangsvariablen und dem Leistungsbild zum Entlasszeitpunkt.

$2 \mathrm{~d}$ Es bestehen Zusammenhänge zwischen den Zugangsvariablen und der Abstinenzrate zum Katamnesezeitpunkt.

$2 \mathrm{e}$ Es bestehen Zusammenhänge zwischen den Zugangsvariablen und der Erwerbssituation nach einem Jahr. 


\section{Material und Methoden}

\subsection{Themenbetrachtung, Hintergrund}

In den Denksystemen evidenzbasierter Medizin werden in allen medizinischen Bereichen kontinuierlich katamnestische Erhebungen erstellt. Im Rahmen vergleichender Betrachtungen wird versucht, aus komplexen therapeutischen Settings und Abläufen einzelne Wirkfaktoren zu isolieren. Diese Wirkfaktoren, bzw. Kombinationen aus diesen Wirkfaktoren, sollen die Grundlage für kompetente Behandlungsstandards sein. Ziel ist es also, über statistische Auswertung und klinische Erfahrung möglichst exakt und gut objektivierbar, die Elemente eines therapeutischen Behandlungsablaufes zu definieren, die für spätere Therapien prädiktorisch nutzbar sein können.

Im Bereich der Psychiatrie, Psychosomatik und der Suchtbehandlung ist dieses Herausschälen von einzelnen Wirkfaktoren häufig nur schwer möglich. Gerade in einem ganzheitlichen Behandlungsansatz, in dem nach dem biopsychosozialen Krankheitsmodell (Feuerlein 2008) die Funktionen aller Lebensbereiche erfasst, reflektiert und bearbeitet werden sollen, ist die Bewertung einzelner therapeutischer Elemente oft sehr subjektiv. In therapeutischen Gefügen, wie sie sich in einer Suchtrehabilitation ergeben, geschieht über einen Zeitraum von beispielsweise 16 Wochen soviel an intensiver Begegnung und damit auch Übertragung sowie Gegenübertragung, dass einzelne Beziehungsstränge in ihrer Bedeutung für das therapeutische Outcome immer nur mutmaßlich bewertet werden können.

Ein interessanter Aspekt der sich aus Gesprächen, die z. T. viele Jahre nach erfolgreicher Suchtrehabilitation stattfanden, herausschälte ist der, dass einzelne, ehemalige Patienten aus inrer Therapiezeit zitieren und empfundene Wirkfaktoren benennen. Hierbei ergibt sich ein buntes Bild an nachvollziehbaren Bewertungen, aber auch an höchst erstaunlichen und unerwarteten Gewichtungen. Es wird in solchen Gesprächen deutlich, dass teilweise falsch verstandene oder falsch wiedergegebene Äußerungen für den Betroffenen zum helfenden Schlüsselsatz wurden. Des Weiteren zeigt sich, dass grundsätzliche Aussagen zur Abstinenzsicherung erst nach häufigem Wiederholen von den Patienten verinnerlicht wurden. Hierbei muss keinesfalls der behandelnde Arzt oder Therapeut derjenige gewesen sein, der die „therapeutische Wahrheit" erfolgreich 
aussprach. Vielmehr wird durchaus die Bedeutung von Begegnungen mit Mitpatienten, externen Betroffenen oder von so genannten „Nebenfiguren“ des therapeutischen Personals gelegentlich als besonders prägend beschrieben.

Es ist nach wie vor insgesamt unbestritten, dass korrigierende, therapeutisch wirksame Beziehungen die wichtigsten Behandlungselemente in einer Suchttherapie darstellen. Der Vernetzung dieser Beziehungen dürfte hierbei eine maßgebliche Bedeutung zukommen. Dabei ist im klinischen Alltag oft zu beobachten, dass die organisierte Beziehung zum zuständigen Arzt oder Therapeuten nicht die maßgebliche sein muss. Vielmehr zeigt sich, dass das Zusammenspiel der unterschiedlichen Beziehungen sowie der strukturelle Hintergrund, vor dem diese Beziehungen gelebt werden, als komplexer Hauptwirkfaktor gesehen werden muss.

Weiterhin gilt, dass eine therapeutische Maßnahme, welche auch unangenehme Realitäten abbildet, eher für das Leben nach der Therapie vorbereitet, als eine harmonische und vom Patienten als höchst positiv beschriebene Maßnahme. Demnach sind weniger angenehme und deshalb mit Missempfindungen besetzte Beziehungselemente innerhalb des Gesamtbeziehungsgefüges als äußerst notwendige Frustration zu sehen. Man spricht hier vom Wirkfaktor der Problemaktualisierung oder Problemaktivierung. Sicherlich gilt dies nur dann, wenn die Frustrationen oder Verzichtsleistungen im Gesamtbeziehungsgefüge aufgefangen, abgefedert und bearbeitet werden können (Grawe 2004). Bei der Isolation von wirksamen Therapieelementen dürfte es aber nur schwer möglich sein, eine angemessene Relation von Frustration und ausgleichender Bergung zu definieren. Selbst wenn diese im Sinne der klinischen Erfahrung akzeptiert wäre, so wäre sie in therapeutischen Standards kaum formulierbar. Auch hier zeigt sich die Schwierigkeit bei dem Versuch, verlässliche, wirksame Faktoren einer Suchttherapie allgemein gültig zu benennen.

Des Weiteren gilt, dass die Patientenbewertung einer therapeutischen Maßnahme nur unter großem Vorbehalt die umfassende reale Wirksamkeit abbildet. Aus diesem Grund muss auch der Wunsch eines Patienten, bei der freien Wahl seiner Behandlungseinrichtung hinsichtlich einer positiven Prädiktion, als sehr fraglich gesehen werden. Zumindest sollten die Argumente, die ein Betroffener zur Begründung seines Wahlwunsches ins Feld führt, geprüft werden. Dann müsste auf dem Hintergrund von zu erwartender therapeutischer Relevanz differenziert 
werden, ob der Wahlwunsch eher förderlich oder hinderlich für den therapeutischen Prozess zu sehen ist.

Die Steuerung im Bereich der medizinischen Rehabilitation sollte also nach wie vor durch sachliche Kriterien und nicht alleinig oder primär von Patientenpräferenzen bestimmt werden.

Die Rehabilitationsforschung beschäftigt sich aus diesem Grund seit Mitte der 90er Jahre mit der Detektion und Isolation von Wirkfaktoren, der Formulierung von Behandlungsstandards und der Erstellung von Assessments zur Verbesserung von Zugangs- und Therapiesteuerung.

Inwieweit die Forschungsergebnisse praktisch sinnvolle Zuordnungen und daraus resultierende Verbesserungen des Behandlungsoutcomes erbringen können, muss sicher noch in Langzeitverläufen überprüft werden.

Eine Standardisierung hinsichtlich Strukturqualität, diagnostischer Möglichkeiten und Behandlungskonzept einer Einrichtung sollte unbedingt nach wie vor stattfinden. Die Sanierung der historisch gewachsenen, teilweise unüberschaubar vielfältigen Allokationslandschaft im deutschen Rehabilitationswesen, ist definitiv indiziert. Bei Standardisierung und Normierung besteht mit großer Wahrscheinlichkeit aber auch die Gefahr, dass bei Schwerpunktsetzung von so genannten objektiven Wirkfaktoren die weniger anerkannten Begleitfaktoren in den Hintergrund geraten und damit im therapeutischen Alltag an Bedeutung verlieren. Gerade diese könnten aber im Sinne einer Gesamtschau die evidenten Elemente sein oder die Vorraussetzung für eine evidente Behandlung schaffen.

Aus Sicht des Autors ist es deshalb sinnvoll, zunächst im Bereich der Strukturqualität, der Behandlungskonzepte, der Organisation und der Abläufe die Basisstandards zu formulieren. Der inhaltlich therapeutische Bereich, der in seiner Bewertung hinsichtlich einzelner Wirkelemente nur sehr schwer sicher zu differenzieren ist, sollte deshalb in der Gesamtheit und mit viel Handlungsspielraum für die jeweilige Einrichtung betrachtet werden.

Die hier vorliegende Arbeit möchte vor allem die Fragestellung aufgreifen, inwieweit möglicherweise Verbindungen zwischen dem Zugangsweg zur Rehabilitation und dem therapeutischen Outcome bestehen. Ob unterschiedliche Auswirkungen auf das Therapieergebnis festzustellen sind, wenn ein Patient über Sozialbericht und Verteilung durch den Kostenträger der Rehabilitationseinrichtung zugeordnet wird, ob es einen Unterschied macht, wenn 
er durch ein Nahtlosverfahren vom entgiftenden Krankenhaus direkt in die Langzeitentwöhnung vermittelt wird, ob es sich auswirkt wenn er nach SGB II über eine zuständige Einrichtung der Agentur für Arbeit dem Rehabilitationswesen zugeführt wird oder ob andere Zugangsformen messbare Unterschiede im Behandlungsergebnis erbringen. Zudem sollen regelhaft erhobene Daten in Form von definierten Zugangsvariablen auf ihre Auswirkungen auf das Therapieergebnis und die Nachhaltigkeit des Therapieergebnisses überprüft werden.

Die unterschiedlichen Formen der Rehabilitationszugangsteuerung sind in den meisten Fällen unabhängig von der Problematik der Beeinflussung durch bestehende strukturelle Vorgaben und Beziehungsgeflechte. Therapiewiederholende Patienten oder Patienten, die auf eine gezielte Empfehlung hin eine Einrichtung wählten, sind zwar nicht als völlig neutral zu betrachten, dennoch spielt sich die Wahl der Einrichtung vor Beginn der Therapie ab. Der Zugangsweg zur Klinik kann deshalb relativ losgelöst vom therapeutischen Geschehen gesehen werden. Die Einrichtung, ihre Strukturqualität, ihre personelle Besetzung und ihr therapeutisches Programm können also als eine verhältnismäßig homogene Einheit und damit als ein Gebilde mit hoher Behandlungskonstanz betrachtet werden. Die behandelnde Rehabilitationsklinik kann somit in ihrer Gesamtheit als ein einzelnen Wirkfaktor betrachtet werden.

Die Wissenschaftler der Rehabilitationsforschung wollen die Behandlungsabläufe standardisieren, den Behandlungsbedarf in so genannte RehabilitandenManagement-Kategorien (RMK) klassifizieren und dann durch gezielte Rehabilitationssteuerung in die passenden Kliniken eine Ergebnisverbesserung erreichen.

Ziel der vorliegenden Arbeit ist es also auch, im Bereich bereits vorliegender und vergleichbarer Zugangsvariablen prädiktorische Elemente für ein positives Behandlungsoutcome $\mathrm{zu}$ finden und diese mit bereits bestehenden Forschungserkenntnissen zu vergleichen. Die Datengrundlage bildet, wie bereits erwähnt, der Datenpool des Entlassjahrgangs 2004 der behandelten alkoholabhängigen Patienten des Diakonie-Krankenhauses Harz GmbH in Elbingerode (DKH). 


\subsection{Zugangssteuerung zur Medizinischen Rehabilitation}

Die moderne, evidenzbasierte Medizin versucht, klinische Behandlungspfade zu definieren. Die Verbesserung der Behandlungseffizienz und die Minimierung der Behandlungskosten sind die ausschlaggebenden Leitgrößen. Sowohl im stationären als auch im ambulanten Bereich sollen fachübergreifende Ablaufpläne die Durchführung der medizinischen Behandlung optimieren. In den Bereichen Akutmedizin und rehabilitative Medizin ergeben sich sehr unterschiedliche Aspekte und Schwierigkeiten bei der Festlegung von Behandlungsstandards (Rapp 2008). Stabile Behandlungsqualität und verantwortlicher Umgang mit verfügbaren Ressourcen müssen in den jeweiligen Bereichen medizinischer Behandlung neu geordnet und qualitätssichernd definiert werden (König 2008). Die klare Festlegung der Aufgaben sowie die Sicherung von Durchführung und Ergebnis bilden das Fundament von verbindlichen Therapiestandards und Behandlungspfaden.

Ein schlüssiger Behandlungspfad steuert den Behandlungsprozess und sichert behandlungsbegleitend die Dokumentation. Später stattfindende Evaluationen können dann Vergleichbarkeit und Anpassung mit stetiger Verbesserung der Abläufe gewährleisten. Ein klinischer Behandlungspfad kann zudem Grundlage zur Kostenträgerrechnung darstellen. Durch strukturierte Dokumentation geplanter Behandlungen bzw. Auflistung von verbrauchten Ressourcen, kann eine vorausschauende Plankostenrechnung für weitere Behandlungsabläufe möglich gemacht werden.

\subsection{Forschungsüberblick}

\subsubsection{Bisherige Entwicklungen}

Seit Mitte der 90er Jahre wird im Bereich der Rehabilitationsforschung in vielfältiger Weise der Versuch unternommen, bedarfs- und leistungsbezogene Patientenklassifikationen zu bilden (Lindenmeyer 2008). Ziel war hierbei die Verbesserung der Wirtschaftlichkeit, des Leistungsumfangs und der Behandlungsqualität. Vorhandenes Wissen hinsichtlich guter therapeutischer Behandlung, der so genannte implizite Behandlungsstandard, sollte abgebildet werden. Die Orientierung der Behandlungspläne erfolgte am biopsychosozialen 
Modell der Internationalen Klassifikation der Funktionsfähigkeit, Behinderung und Gesundheit (ICF). Es sollten angemessene, d. h. flexible, Therapiezeiten entstehen. In innovativen Ansätzen sollten Schnittstellenproblematiken einem effizienten Management zugeführt werden. Die Nutzung und Integration ambulanter Leistungsformen sollte stattfinden. Kombinationstherapien mit ambulanten und stationären Behandlungsphasen im Wechsel sollten zum Einsatz kommen. Verknüpfungen zwischen Berufsorientierung und Suchtrehabilitation sollte vermehrt und multilokal stattfinden. Das Stichwort der Passgenauigkeit und Individualität durch differentielle Integration rückte für den Bereich der Therapievorbereitung mehr und mehr in den Mittelpunkt (Kulick 2009). Ein parallel hierzu formulierter Ansatz schlug sich in der Bildung von so genannten Rehabilitanden-Management-Kategorien (RMK) nieder (Spyra et al. 2010). Hier sollten die Fälle mit homogenem Behandlungsbedarf beschrieben werden. Evidenzbasierte Erkenntnisse und klinische Erfahrungen bzw. Expertisen sollten mit einfließen (ausführlicher siehe 2.3.2).

Ein langfristiges Ergebnis der Forschungsarbeiten sollte in der Ordnung und Balancierung einer Rehabilitationslandschaft bestehen, die aus historischen Gründen sehr inhomogen imponiert. Durch uneinheitliche Kostenträgerlandschaft und eine große Vielfalt an Einzelverträgen zwischen Kostenträger und Behandlungseinrichtung, war eine unüberschaubare Menge an Behandlungen, Behandlungsqualität, Ressourcenverwaltung sowie Vergütungsmodellen entstanden.

Ein durchgehend konstantes Element ergab sich durch die Tatsache, dass Behandlungen über die Behandlungsdauer definiert und entsprechend nach Behandlungstagen vergütet wurden.

\subsubsection{Die Rehabilitanden-Management-Kategorien (RMK)}

In der Weiterentwicklung der oben beschriebenen Bereiche der Rehabilitationsforschung wurde deutlich, dass speziell in der Suchtrehabilitation Verbesserungen der Behandlungsmöglichkeiten, in der Optimierung von Schnittstellen und in der Erhöhung der grundsätzlichen Patientenzufuhr ins versorgende System, zu finden sind. 
Dem gegenüber dürfte die Erweiterung und Verfeinerung des therapeutischen Angebots der einzelnen Rehabilitationseinrichtungen derzeit als untergeordnet zu sehen sein.

Für die Alkoholabhängigkeit sollen zukünftig Therapiestandards in der Rehabilitation eingeführt werden, die im Expertenkonsens erarbeitet wurden. Sie sind in einer Pilotversion der Ludwig-Maximilian-Universität München über Therapiestandards bei Alkoholabhängigkeit zusammengefasst (Soyka et al. 2009). Diese Standards beinhalten die Definition für ein Mindestmaß an therapeutischer Leistung in der stationären und ganztägig ambulanten Therapie.

Über Qualitätssicherung und Katamneseuntersuchungen werden Erfolge der stationären Entwöhnungsbehandlung Alkoholabhängiger belegt.

Eines der Hauptprobleme bei der Behandlung von Abhängigkeitserkrankten ist, dass ein zu geringer Teil der Patienten trotz genehmigter Entwöhnungstherapie diese nicht antritt. Die prozentualen Angaben über diese so genannten Nichtantreter schwanken in der Literatur im Regelfall zwischen $10 \%$ und $30 \%$. In einer Studie über innovative Elemente in der Entwöhnungsbehandlung wird bezüglich der Vermittlungsverluste durch ungünstiges Schnittstellenmanagement eine erschreckende Aussage gemacht. $50 \%$ der Antragsteller einer Langzeitentwöhnungsmaßnahme würden sich im unüberschaubaren Netz der Angebote verfangen und letztlich die geplante Maßnahme nicht antreten (Kulick 2009). Die hinsichtlich dieses Aspekts dokumentierten Zahlen der Klinik, in der die vorliegende Untersuchung durchgeführt wurde, befinden sich im oben beschriebenen Durchschnittsbereich. Bei dem diesbezüglich eingehend untersuchten Jahrgang 2010 ergibt sich beispielsweise ein Anteil von nicht angetretenen Patienten von 26,6\%. D. h. 188 von 706 einbestellten Patienten haben die Therapie nicht begonnen (klinikinterne Auswertung).

Ein weiterer Aspekt bei der Frage nach der Optimierung von organisatorischen Abläufen bei der Behandlung Abhängigkeitserkrankter und der Therapie selbst ist der, dass die Möglichkeiten der vorhandenen, unterschiedlichen Therapiesettings oft noch nicht ausgeschöpft oder nicht maximal kombiniert werden. Die Projektergebnisse der Deutschen Rentenversicherung des Jahres 2009 im Land Rheinland-Pfalz wiesen aus, dass indikative Verknüpfung von Leistungselementen, Förderung von sozialer und beruflicher Stabilität und verbesserte Koordination im Gesamtrehabilitationsprozess an Bedeutung weiter zunehmen werden (Rische 2009). 
Die krankheitsspezifische, komplexe Erfassung und Integration der Therapiesteuerung und der Therapieplanung muss unbedingt gewährleistet sein. Bei unzureichender Berücksichtigung der Interaktionen der einzelnen Erkrankungen bei Komorbiditäten, kann z. B. bei Abhängigkeitserkrankungen zu einer Gefährdung des Gesamtprozesses führen (Vogelsang 2009). Insbesondere psychische Begleiterkrankungen, wie Depressionen oder Angststörungen, verstärken die Problematik der Abhängigkeitserkrankung erheblich und verursachen weitere Hemmnisse, die die Abstinenz und die soziale bzw. berufliche Reintegration zusätzlich erschweren. Auch die Gewichtung der einzelnen Krankheitskomponenten muss bei der Therapieplanung und der Rehabilitationssteuerung angemessen durchgeführt werden. Phasenweise werden bedeutsame Komorbiditäten nicht berücksichtigt und trotz gutem Behandlungsansatz hinsichtlich der Haupterkrankung und evtl. anderer Nebenerkrankungen, kann der Gesamterfolg dadurch gefährdet werden.

Da Sucht im Sinne des biopsychosozialen Krankheitsmodells eine ganzheitliche Störung ist, bei der alle Lebensbezüge betroffen sind, muss die Komorbidität unbedingt berücksichtigt werden. Da diese sowohl einen erneuten Rückfall bedingen kann, als auch evtl. ursächlich als Auslöser bzw. Entstehungsursache der Abhängigkeitserkrankung überhaupt verstanden werden muss, bekommt die Komorbidität somit Bedeutung im Sinne einer möglichen Wurzel für das gesamte Krankheitsgebilde.

Bei der Behandlung von stofflichen Süchten wird aber die Hauptdiagnose und damit die Zuweisungsdiagnose immer über den stofflichen Konsum definiert. Bei einem komplexen Erkrankungsbild mit Komorbiditäten sollte deshalb dringend die Behandlungspriorität überprüft werden. Wenn im Rahmen der synergistischen Prinzipien die individuelle Kausalwirklichkeit des Patienten nicht ausreichend berücksichtigt wird, ist sowohl von einer suboptimalen Motivation des Betroffenen auszugehen, als auch eine fragliche Wirksamkeit des Behandlungsansatz zu erwarten.

Aus den Untersuchungen der Deutschen Rentenversicherung Bund aus dem Jahr 2008 lässt sich entnehmen, dass mit 22.000 Rehabilitationsleistungen für Abhängigkeitserkrankungen der Sektor Suchtrehabilitation die 4. Stelle der erbrachten Rehabilitationsleistungen der DRV Bund einnimmt (Egner et al. 2009). Hieraus wurde deutlich, dass die Steuerung der Rehabilitation, d. h. letztlich der Rehabilitationszugang, als wichtiger erster Schritt gesehen werden muss, der 
individuell und indikativ auf den Rehabilitanden zugeschnitten werden sollte. Die bekanntermaßen angefertigten Sozialberichte dienen nach wie vor als fachliche Information für den sozialmedizinischen Dienst der Deutschen Rentenversicherung Bund. Dieser soll daraufhin den Rehabilitationsbedarf differenziert feststellen und eine möglichst geeignete Einrichtung für den Rehabilitanden und seinen Rehabilitationsprozess auswählen. Falls der Sozialbericht, oder eine vergleichbare Stellungnahme, aus anderen Verfasserquellen kommt, z. B. von niedergelassenen Ärzten, von Krankenhäusern, von Sozialdiensten anderer Einrichtungen etc., bleibt die kompetente Zugangssteuerung unverändert.

Da der sozialmedizinische Dienst in diesem Falle die entscheidende Instanz für die Zuordnung darstellt, ist es zwingend notwendig, dass an dieser Stelle der individuelle Behandlungsbedarf für einen Patienten definiert wird.

Der spezifische Rehabilitationsbedarf eines einzelnen Rehabilitanden erfordert medizinisch-therapeutische Konzepte, die die modernen Behandlungsstandards mit einem angemessenen $\mathrm{Ma}$ an Individualisierung verbinden, um somit die grundsätzlichen Rehabilitationsziele; Abstinenz und Erwerbsfähigkeit; zu erreichen. Diese Zielerreichung muss im Rehabilitationsprozess maßgeblich sein und dementsprechend Dauer und Verlauf der Leistung bestimmen. Damit wird die Behandlungsdauer zur flexiblen Größe. Zu einer jeweiligen Grundbehandlungszeit sind Verkürzungen oder Verlängerungen möglich. Beim weiterentwickelten Ansatz der Rehabilitationsforschung, der Fallgruppenbildung in der Rehabilitation aus den Rehabilitanden-Management-Kategorien, wird also über den Behandlungsbedarf und die Therapiedauer reguliert.

Die Projekte des Lehrstuhls für Versorgungssystemforschung und Grundlagen der Qualitätssicherung in der Rehabilitation an der Charité-Universitätsmedizin Berlin versuchen homogene Behandlungsgruppen und daraus resultierend eine individuell passende Therapiesteuerung durch die Nutzung von Therapieorientierungswerten zu erreichen. Hierzu sollen Rehabilitanden mit gleichartigem Behandlungsbedarf und vergleichbaren therapeutischen Leistungen in Fallgruppen zusammengefügt werden. So sollen spezielle Bedarfslagen von Patientengruppen innerhalb einer Indikation identifiziert und mit den Möglichkeiten des Leistungserbringers abgeglichen werden (Spyra et al. 2010).

Die Bedarfsgruppierung leitet sich zunächst aus der rehabilitationstypischen Hauptindikationsgruppe (z. B. Suchtrehabilitation) ab. Innerhalb der 
Hauptindikationsgruppe wird dann nach häufig vorkommenden und klinisch relevanten Diagnosengruppen (z. B. Alkoholabhängigkeit) gemäß der internationalen Klassifikation der Krankheiten (ICD) differenziert. Daraufhin erfolgt die Unterscheidung der Bedarfsgruppen nach dem rehabilitationsrelevanten Schweregrad der Beeinträchtigung unter Berücksichtung der somatischen, psychischen und sozialen Dimension. Grundlage der Beurteilung bietet das biopsychosoziale Behinderungsmodell der internationalen Klassifikation der Funktionsfähigkeit, Behinderung und Gesundheit (ICF).

Neben der Bedarfsgruppencharakteristik werden für die RehabilitandenManagement-Kategorien (RMK) auch die bedarfsgruppenspezifischen Behandlungsanforderungen definiert. Diese betreffen zunächst die therapeutischen Leistungen. Zukünftig sollen sie auch die medizinische Diagnostik, die ärztlichen und pflegerischen Leistungen sowie die Medikation umfassen.

Der bedarfsorientierte Ansatz der Fallgruppenbildung ermöglicht es, die RMK in der Zugangssteuerung beim Kostenträger einzusetzen. Dem sozialmedizinischen Dienst wird über eine mögliche Zuordnung der Versicherten zu einer Bedarfsgruppe im Rehabilitationsantragsverfahren eine Orientierungshilfe zur Verfügung gestellt, die besondere Bedarfslagen anzeigt und inn bei der Auswahl einer geeigneten Behandlungseinrichtung unterstützen kann (Abbildung 1).

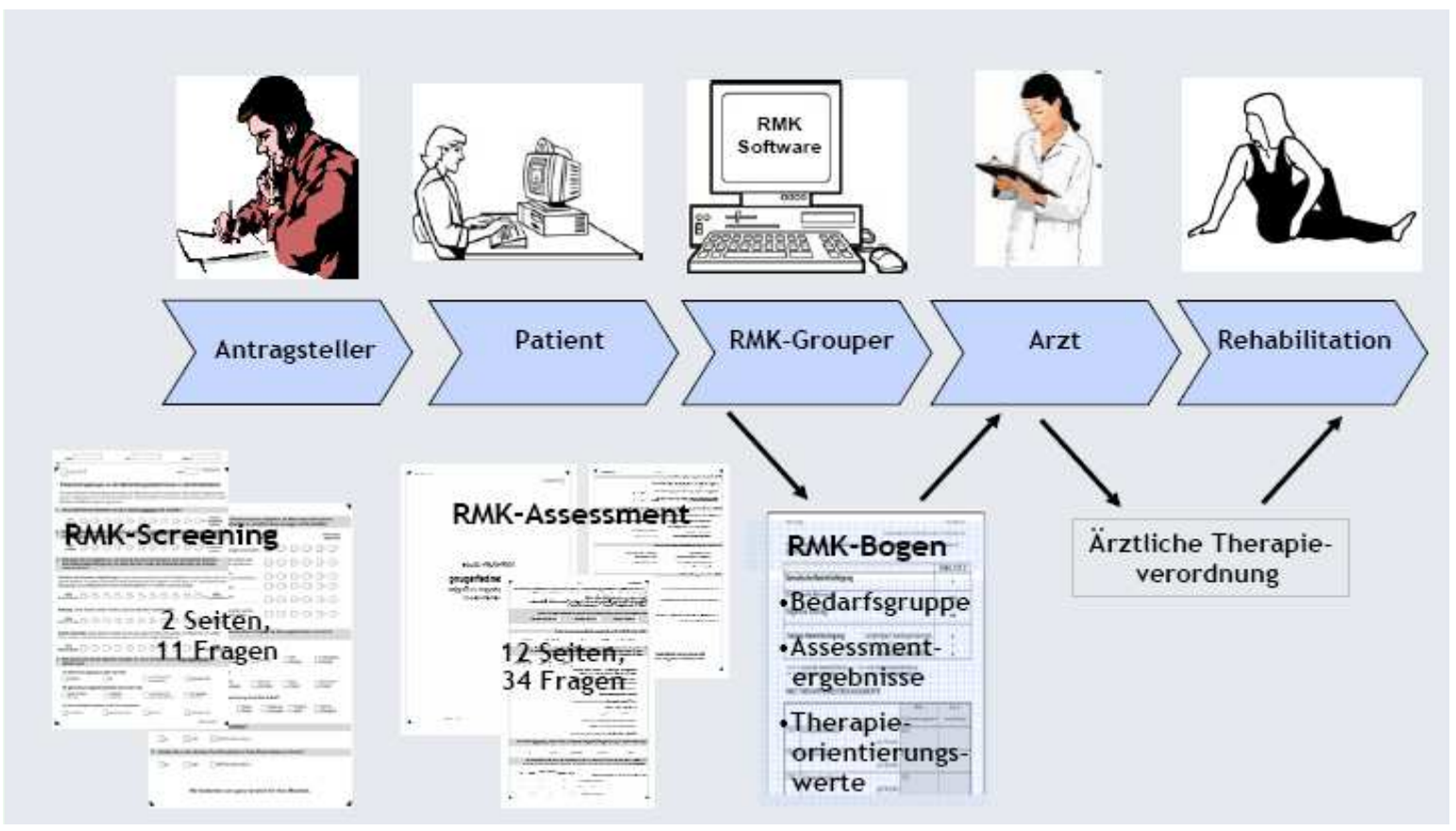

Abbildung 1: Rehabilitanden-Management-Kategorien(RMK)-Projekt:

Verfahrenswege nach Spyra et al. 2010 
Somit soll bereits durch eine optimierte Zugangssteuerung die Wahl der Therapieeinrichtung verbessert werden. Dadurch ist davon auszugehen, dass die Effektivität der Rehabilitationsmaßnahme erhöht wird. Innerhalb der Rehabilitationseinrichtung können die RMK ebenfalls eingesetzt werden, und zwar zur Steuerung des Therapieprozesses. Neben der Bedarfsmessung kommen dann auch definierte Behandlungsanforderungen, die sich am spezifischen Bedarf der Fallgruppe ausrichten, zum Tragen. Die Zuordnung der Rehabilitanden zu einer Bedarfsgruppe kann den behandelnden Arzt der Behandlungseinrichtung bei seinen Entscheidungen unterstützen. Durch verbesserte Transparenz der Prozesse und Auskunft über die angebotenen Leistungen der Rehabilitationseinrichtung, wäre eine bessere Vergleichbarkeit der Kliniken untereinander gegeben (Benchmarking), was ebenfalls den Rehabilitanden zum Vorteil gereichen könnte.

Ein RMK-Assessment, welches in der Charité im Rahmen eines Forschungsprojektes für den Einsatz in der stationären Entwöhnungsbehandlung bei Alkoholabhängigkeit entwickelt wurde, ist in der Lage, verschiedene Bedarfskonstellationen bei alkoholabhängigen Patienten zu identifizieren.

Es konnten vier Bedarfsgruppen mit unterscheidbarem Behandlungsbedarf in steigender Intensität entwickelt werden. Die Bedarfsgruppen erfassen die psychische und die soziale Dimension und gewichten zwischen auffällig und stark auffällig. Das RMK-Assessment ist für den Einsatz in der Therapieplanung und steuerung konzipiert und steht den Rehabilitationseinrichtungen in Form eines Patientenfragebogens zur Verfügung. Das RMK-Assessment Sucht umfasst 12 Seiten, 34 Fragen mit 220 Items und kann im Mittel in 20 bis 30 Minuten vom Patienten ausgefüllt werden. Die aus den Assessmentdaten gewonnenen Bedarfsgruppen müssen in einer „klinischen Übersetzung“ in den therapierelevanten Behandlungsbedarf des Patienten übertragen werden. Die Behandlungsanforderungen für die einzelnen Bedarfsgruppen basieren auf den Rehabilitationstherapiestandards (RTS), der Leitlinie für stationäre und teilstationäre Rehabilitation bei Alkoholabhängigkeit der Deutschen Rentenversicherung Bund. Bei der Zuordnung der Patienten in die 4 Fallgruppen finden folgende sechs Subdimensionen Berücksichtigung: „substanzenbezogene Beeinträchtigung“, „psychische Symptomatik“, „persönliche Ressourcen“, 
„arbeitsbezogenes Erleben und Verhalten“, „Alter und Arbeitslosigkeit“, „,soziale Unterstützung“ (siehe Abbildung 2).

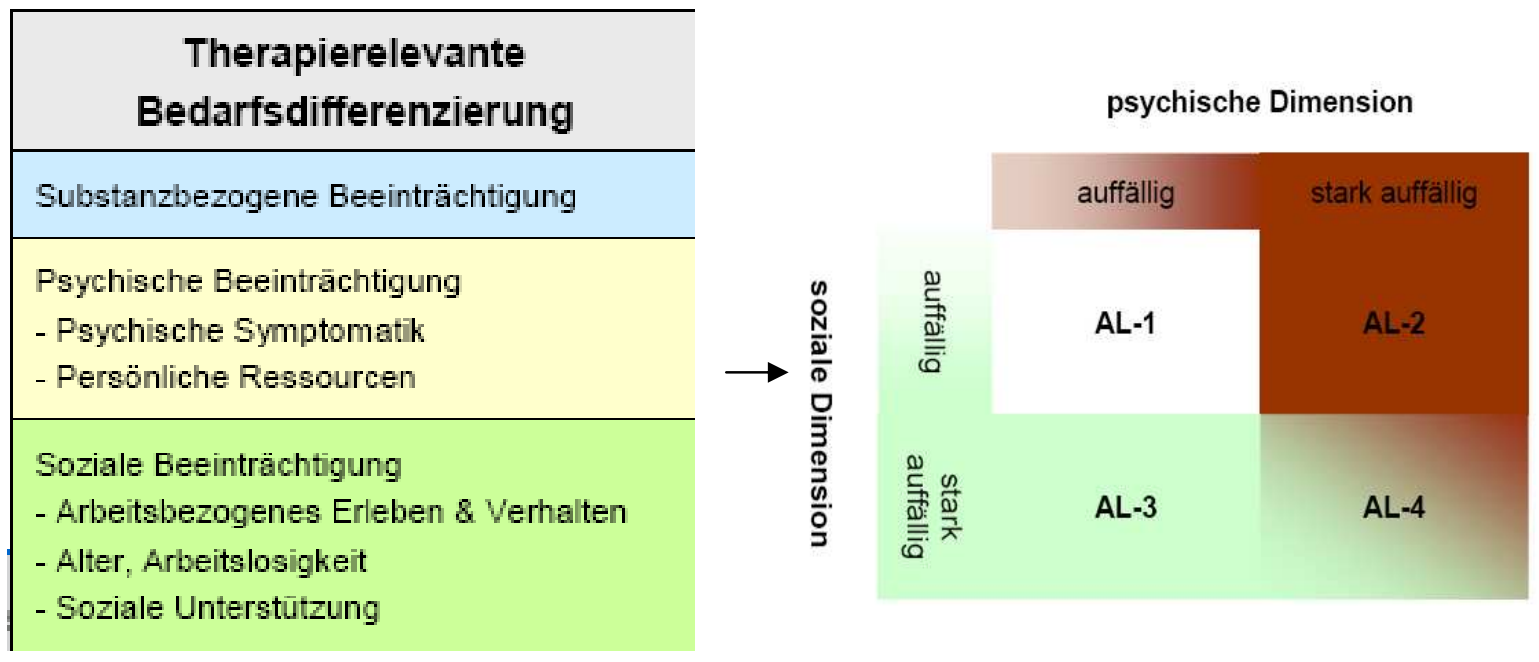

Abbildung 2: Fallgruppenbildung aus 6 Sub-Dimensionen der therapierelevanten Bedarfsdifferenzierung nach Spyra et al., 2010 Legende:

AL-1 bis AL-4: die resultierenden Bedarfsgruppen für alkoholabhängige Patienten

Die Angemessenheit der Zuordnung liegt in den meisten Fällen deutlich über $75 \%$. Gleichzeitig ist eine 100\%ige Sicherheit in der Zuordnung sehr unwahrscheinlich. Dies ist darin begründet, dass die Zuordnungsklassen nicht vollständig, sondern nur statistisch hinreichend, homogen sind. Ein stabiler Zusammenhang ergab sich aus der Schweregradausprägung und der Gesamtdauer der Rehabilitationsmaßnahme.

In den Projektkliniken wurden sowohl seitens der Rehabilitanden als auch des medizinischen Personals eine positive Beurteilung abgegeben. Der RMK-Bogen wurde als nützlicher Baustein für Diagnostik und Therapieplanung empfunden.

Für die Rehabilitationsteuerung, d. h. für die Zuordnung eines Patienten in die optimale Klinik, wurde ein standardisiertes RMK-Assessment in Kurzform entwickelt. Es entspricht einem Screeningverfahren, welches aus 20 Items besteht. Durch diese werden die Beeinträchtigungsdimensionen abgebildet. Hierdurch werden die 4 Bedarfsgruppen dargestellt.

Die Zugangssteuerung wurde damit für den zuweisenden Arzt erleichtert (Spyra et al. 2010). 


\subsection{3 Überblick über Studienergebnisse der Rehabilitationsforschung}

In allen nicht-suchtspezifischen Behandlungsbereichen waren Suchtprobleme eine absolute oder relative Kontraindikation für Rehabilitationsprozesse und verhinderten somit häufig den Zugang in stabilisierende Behandlungsangebote. Suchtkranke weisen nun, sozusagen primär krankheitsimmanent, ausgeprägte strukturelle Defizite und viele Behandlungshemmnisse auf. Entsprechend ist die Steuerung der Rehabilitation im Bereich Suchterkrankungen massiv erschwert und verglichen mit anderen Fachbereichen überdurchschnittlich kompliziert zu gestalten.

Die Aufsichtsfunktion und Qualitätssicherung der Deutschen Rentenversicherung hatte eine Weiterentwicklung der Behandlungskonzepte und ihrer Umsetzung zur Folge: Leistungen und Ergebnisse wurden in Beziehung gebracht, Datenquellen konnten erschlossen werden, Umsetzungen in Strukturanforderungen wie Ausstattung oder Personalqualifikation wurden erreicht. Bei der Standardisierung von Konzepten, als Basis für Vergleich und Überprüfung, muss allerdings in breiten Kategorien gedacht werden. Die individuelle Komponente innerhalb einer Einrichtung steht sonst in der Gefahr, zu eingeengt oder verdrängt zu werden (Funke 2009).

Ergebnisse aus der Qualitätssicherung der Suchtrehabilitation der Deutschen Rentenversicherung (Beckmann et al. 2009) erbrachten, dass die kontinuierliche Weiterentwicklung und Aktualisierung sinnvolle Weiterentwicklungsergebnisse aufweisen.

Seit 1994 wird die Qualitätssicherung der stationären Rehabilitation kontinuierlich aktualisiert und erweitert. Seit 1997 ist das Peer Review Verfahren eingeführt. 2007 wurde die überarbeitete Klassifikation therapeutischer Leistungen installiert. In den letzten Jahren wurden im Rahmen des Rehabilitationsleitlinienprogramms der Rentenversicherung auf wissenschaftlicher Grundlage Behandlungsstandards für die Rehabilitation entwickelt. Deren Implementierung hat im Jahr 2009 begonnen. Diese Behandlungsstandards wurden zuerst evidenzbasierte Therapiemodule (ETM) genannt. Da aber auch Behandlungsmaßnahmen integriert wurden, die nicht den Kriterien der evidenzbasierten Medizin entsprachen, sondern lediglich den Maßstäben der „best practice“, wurden sie in 
Form von so genannten Rehabilitationstherapiestandards

zusammengefasst.

Die Qualitätskennzahlen für eine Rehabilitationseinrichtung werden aus dem Peer Review Ergebnis, der Rehabilitandenzufriedenheit, der adäquaten Durchführung und Dokumentation therapeutischer Leistungen und dem subjektiven Behandlungserfolg gebildet.

Weitere Forschungen im Bereich Qualitätssicherung finden ständig statt; im Jahr 2010 wurde der Einsatz eines Patientenfragebogens für die Suchtrehabilitation sowie die Veröffentlichung von einheitlichen Strukturanforderungen der Rentenversicherung für stationäre Rehabilitationseinrichtungen begonnen.

Eine klinische Studie über Patientenfallgruppen in der medizinischen Rehabilitation am Beispiel der Behandlung von Alkoholkranken (Lindemeyer 2008) untersuchte die Zuordnungsmöglichkeiten in Patientenkategorien. Hierzu wurden anhand der Eingangs- und Verlaufsdaten von 2.217 stationär behandelten Alkoholabhängigen aus vier stationären Entwöhnungseinrichtungen Vergleiche angestellt.

In dieser umfangreichen Klinikstudie konnten drei Fallgruppen identifiziert und klinikübergreifend einem individuellen, akzentuierten Behandlungsbedarf zugeordnet werden. Die Komplexität der im Einzelfall vorgenommenen Indikationsentscheidungen, hinsichtlich der Gewichtung von psychotherapeutischen, sozialtherapeutischen und medizinischen Behandlungsangeboten, konnte dabei herausgearbeitet werden. Das traditionelle und in der Praxis etablierte Ziel einer Komplexbehandlung, die diese drei Bereiche berücksichtigt und zudem berufsfördernde Maßnahmen integriert, sollte umgesetzt werden.

Die Standardisierung und Vergleichbarkeit, aber auch die Leistungsdifferenzierung, die den individuellen Bedürfnissen des Patienten entsprechen, sollten Berücksichtigung finden.

Die Unterschiedlichkeiten der Settings und der Behandlungszeiten, die Differenzierung bei unterschiedlicher Krankheitsschwere und die Komorbiditäten flossen in die Bewertung ein.

Die Personalausstattung der Einrichtungen wurde normiert.

Die Flexibilisierung spiegelte sich vor allem im Bereich der vernetzten Behandlungsweise wider. Einzel-, Gruppen- und Kombinationstherapie kamen zur 
Anwendung. Die Standardelemente definierten ein Mindestmaß von Behandlungen. Hinzu kam ein Mix mit flexiblen, patientenspezifisch individuellen Behandlungselementen.

Die Kostenberücksichtigung fand dergestalt statt, dass zunächst die günstigste Behandlungskategorie angewandt wurde. Erst beim Scheitern kam die finanziell nächst höhere Behandlungsstufe zum Tragen (Stepped care). Die gezielte Allokation vereinte die Elemente Indikation, Therapieinhalte, Therapiedauer, Behandlungssetting, Therapieintensität, notwendige Ressourcen und spezielle Indikation.

Innerhalb der Therapie fand eine Prozessanpassung, d. h. die verlaufsabhängige Prozessdifferenzierung, statt. Die Organisation bzw. die Bahnung von Nachsorge, Wieder- oder Weiterbehandlung wurde erarbeitet.

Bei klinisch und ökonomisch optimaler Allokation erwies sich das breit angelegte Therapieangebot mit spezifischer Zuordnung als sinnvollste Variante. Zur Allokationssicherung und Stabilisierung fanden auch Standardfaktoren ihre Berücksichtigung. Unter Einbeziehung von evidenzbasierten Erkenntnissen wurde durch Zusammenfassung von Behandlungselementen in Rehabilitationstherapiestandards (RTS) eine Behandlungsnorm geschaffen. Für diese bildeten die von der Arbeitsgemeinschaft der wissenschaftlichen medizinischen Fachgesellschaft (AWMF) formulierten Behandlungsleitlinien zur Behandlung von Alkoholkranken die konzeptionelle Grundlage (Geyer et al. 2006).

Bei der Überlegung, ob Fallgruppen für die Steuerung der medizinischen Rehabilitation sinnvoll sein könnten, war auch die Problematik der vielfältigen Kostenträger zu bedenken. Inwieweit ein solches System deshalb trägerübergreifend bzw. bundesweit installiert werden kann, ist sicherlich noch nicht abschließend geklärt.

Im Vordergrund der Fallgruppenforschung sollte aber die bedarfsbezogene Leistungsanforderung stehen. Ob es für eine homogene Vergütung eingesetzt werden kann, bleibt ebenfalls abzuwarten. Wenn als Ziel lediglich die Erstellung pauschalisierter Vergütungssysteme angestrebt worden wäre, hätte dies auch unabhängig von Fallgruppen realisiert werden können. Die reine Reduktion der Verweildauer, wie im DRG-System angestrebt, oder die Kreation von Pauschalangeboten mit stabiler, unveränderbarer Gesamtbehandlungszeit, wäre ebenfalls relativ einfach zu konzipieren gewesen. (Keck et al.2008) 
Bei der Fallgruppenbildung, die den therapeutischen Bedarf für Patientengruppen bündeln, ist es sowohl denkbar, dass die Effizienz der Behandlung, aber auch eine Verbesserung der Zugangssteuerung durch den Kostenträger erreicht wird. Zudem wäre möglicherweise bei einem Patientengut mit homogenem Behandlungsbedarf, auch die interne Therapiesteuerung durch den Leistungserbringer während der Rehabilitationsmaßnahme erleichtert. Abläufe könnten besser planbar und klarer nachvollziehbar erstellt werden. Die Bereitstellung von Strukturqualität wäre dadurch verlässlicher. Die Fallgruppe wäre dann ein Instrument für externes und internes Qualitätsmanagement. Die ökonomische Homogenisierung würde zwangsweise folgen.

Wenn man die Installation von Fallgruppen nicht primär unter dem Aspekt einer homogenisierten Vergütung betrachtet, sondern das Augenmerk vor allem auf den Schwerpunkt der bedarfsbezogenen Leistungsanforderung legt, ergibt sich implizit die Notwendigkeit, Behandlungsstandards in der medizinischen Rehabilitation festzulegen.

Eine Fallgruppe muss also sowohl hinsichtlich des Patientenbedarfs, als auch hinsichtlich der hierzu passenden einrichtungsbezogenen, individuellen Behandlungsindikation beschrieben werden. Erst wenn Einrichtungen hierdurch in ihrer Behandlungsmöglichkeit vergleichbar geworden sind, bzw. erst wenn die Unterschiede zwischen den Einrichtungen herausgearbeitet wurden, können Patienten einer Fallgruppe durch gezielte Rehabilitationssteuerung optimal einer Einrichtung zugeordnet werden.

Die Definition von Behandlungsstandards als Grundvoraussetzung für eine evidenzbasierte Medizin ist unumstritten. Ein Katalog für sinnvolle zusätzliche Behandlungsmaßnahmen ist im Bereich medizinische Rehabilitation ebenfalls unerlässlich, da die individuellen Behandlungsaspekte zur Wiederherstellung funktionaler Gesundheit, wie in der ICF klassifiziert, unabhängig vom Hauptkrankheitsbild, starke individuelle Unterschiede aufweisen. Die Behandlungselemente dieses Zusatzkataloges müssten störungsspezifisch zu zuordnen und in ihrer Wirksamkeit gesichert sein. Fachübergreifende Kenntnisse von gültigen Therapiestandards müssen also in die medizinische Rehabilitation einfließen und zur Anwendung gelangen. Dies ist sowohl bei der Konzipierung von Fallgruppen, als auch innerhalb der Behandlungspläne zu berücksichtigen. Hinsichtlich der störungsspezifischen Zuordnung muss also die Frage geklärt sein, ob die Beurteilungskriterien, die aus einem Antrag für medizinische Rehabilitation 
entnommen werden können, ausreichend stabile Daten liefern, um damit eine kompetente Allokation über Fallgruppen möglich zu machen.

Trotz intensiver Forschung, teilweise mit enormem Aufwand, liegen nur sehr begrenzt verwertbare Erkenntnisse vor, die bei der Allokationsentscheidung hilfreich sein können. Nach wie vor besteht die Gefahr der Zirkularität des Allokationsprozesses. Zirkularität beschreibt in diesem Zusammenhang die Tatsache, dass die Allokationsentscheidung vor dem Hintergrund des bestehenden Behandlungsangebotes einer Einrichtung stattfindet. Sie wird also nicht von den individuellen Bedürfnissen des Patienten bestimmt, sondern entsteht in einem Kreislauf von sich selbst bestätigenden Notwendigkeiten der Behandlung. Diese zirkulierenden Prozesse können nur durch äußere Supervision und externe Vergleiche erkannt und überprüft werden, da sie systemimmanent sind und dadurch der Selbstreflexion nicht zugänglich werden. Letztlich führt dies also dazu, dass immer wieder Gewohnheitsabläufe und subjektive Erfahrungen abgebildet werden (Lindenmeyer 2008).

Die Unterschiede, die zwischen verschiedenen Behandlungseinrichtungen festzustellen sind, sind deshalb nicht sicher evidenzbasierte Unterschiede. Vielmehr scheint die vorgehaltene Struktur die maßgebliche Rolle zu spielen. Eine allgemein gültige Evidenz hinsichtlich eines Krankheitsbildes kann also nicht von den regionalen Umständen losgelöst definiert werden. Erfolg oder Misserfolg in der Behandlung ist damit nie sicher einer Einrichtung und ihrem Behandlungsangebot, oder im Gegensatz dazu der Patientenpopulation, zu zuordnen.

Bei der Kreation eines sinnvollen Settings muss berücksichtigt werden, dass Suchtpatienten bezüglich Behandelbarkeit grundsätzlich eine schlechtere Motivationslage aufweisen, als Rehabilitationspatienten anderer medizinischer Bereiche.

Das Setting bei Suchtpatienten muss einerseits einladend und bergend sein, andererseits darf es nicht zu sehr versorgend oder gar verwöhnend sein, da dies dem Störungsbild des Suchtkranken, der pathologischen Selbstversorgung, Vorschub leisten würde. Ein wesentlicher Teil der Behandlung ist deshalb die Reduktion der Bedürfniserfüllung. Das bedeutet aber Verzichtsübung und Umgang mit Unlust sowie Frustration mit dem Ziel, die Frustrationstoleranz für die Zeit nach der Therapie zu trainieren und zu stärken. 
Hier ist klug balancierend abzuwägen. Einerseits kann nur der Patient behandelt werden, der eine Einrichtung aufsucht und auch dort bleibt, d. h. der ein gewisses $\mathrm{Maß}$ an Wohlfühlqualität erlebt, andererseits ist Sichwohlfühlen definitiv nicht Zweck der Suchttherapie, sondern vielmehr Persönlichkeits- und Verhaltensänderung, also durchaus Unlust produzierende Vorgänge.

Die Vorlieben eines Patienten sind zwar im Bereich Qualitätsmanagement wichtige und inzwischen stabil integrierte Faktoren, sie können aber gerade bei Krankheitsbildern mit hohem Krankheitsgewinn und damit gedrosselter Veränderungsbereitschaft des Betroffenen keine zufriedenstellende und ausreichende Grundlage für die Allokationsentscheidung bilden.

Der Grossteil der Patienten richtet sich zudem nicht an der wissenschaftlichen Evidenzforschung aus. Die subjektive Wahrnehmung des Patienten spielt bei der Beurteilung einer therapeutischen Maßnahme die entscheidende Rolle. Alternative Behandlungsmethoden, sehr teure Medikamente oder besondere, moderne diagnostische Verfahren sind bei Patienten häufig sehr beliebt, weil sie durch die Medien verbreitet und damit allgemein bekannt sind. Evidenzbasierte Entscheidungsparameter werden dagegen wenig berücksichtigt. Informationen aus der Populärpresse, Modeerscheinungen und laienhafte Kausalzusammenhänge bilden oft das innere Behandlungskonzept eines Patienten.

Gemäß den hier getätigten Betrachtungen und den klinischen Erfahrungen wird deutlich, dass bei suchtkranken Patienten die Allokationsentscheidung durch dessen Mitbestimmung im Sinne des Shared Decision Makings (Scheibler et al. 2003) nicht sicher verbessert werden kann. Die Entscheidungsmündigkeit des Suchtkranken verändert sich massiv, je nachdem, in welcher Phase des Konsums, der Krankheitseinsicht, der Veränderungsbereitschaft, der Verzichtsbereitschaft und der körperlichen, geistigen und seelischen Stabilität er sich befindet. Man kann sagen, dass suchtkranke Menschen in der Gefahr stehen, durch zeitlich lang andauernden und mengenmäßig ausgeprägten Konsum von Suchtmitteln, ihren Realitätsbezug teilweise so stark einzubüßen, dass innen über weite Strecken die innere Ordnung fehlt, um für sich selbst verantwortlich sorgende und lebenserhaltende Entscheidungen zu treffen. Das emotionale Verlangen nach Entlastung, Erleichterung und Wohlgefühl kann so sehr im Vordergrund stehen, dass hierfür der Preis der Selbstzerstörung durch das Suchtmittel in Kauf 
genommen wird. Dies geschieht, je nach Krankheitsphase, sowohl unbewusst als auch mehr und mehr bewusst. Wichtig ist in diesem Zusammenhang, dass der Betroffene nicht ausreichend frei gegen die zunehmende Fehlentwicklung, und damit Selbstzerstörung, Entscheidungen treffen kann. Aus diesem Grunde wird es unumgänglich sein, dass in einigen Phasen der Abstinenzerlangung, bzw. für einige Entscheidungen auf dem Weg zur Abstinenz, die paternalistische Beziehung zwischen Arzt bzw. Therapeut und Patient vorherrschen muss. Im Laufe der Genesung und Abstinenzsicherung soll dann selbstverständlich eine maximale Mitentscheidung des Patienten erreicht werden. Im Regelfall dürfte also sicherlich ein Shared Decision Making im Verlauf der therapeutischen Maßnahme zunehmend möglich werden. Da die Allokationsentscheidung, in welcher Klinik die Behandlung stattfinden soll, aber am Anfang eines Behandlungsablaufs steht, wird der Großteil der Patienten aus der eigenen Bedürfnislage heraus überfordert sein, die therapeutisch wirksame von der primär emotional-entlastenden Maßnahme zu unterscheiden. Das freie Wahlrecht der Behandlungsstätte muss also im Bereich der Suchterkrankungen, aus Gründen der paternalistischen Verantwortung, dringend hinterfragt werden.

Die Entscheidungsmündigkeit, das freie Wahlrecht der Behandlungsstätte und andere Patientenpräferenzen sind also bei Patienten mit Suchterkrankungen in die Allokationsabwägung, ob Versorgung und Wohlgefühl oder Frustration und belastende Auseinandersetzung in den Vordergrund zu stellen sind, zu integrieren.

Auch ist die Allokationsentscheidung nicht sicher aus der Haltequote zu treffen. Einerseits kann diese ein Indikator dafür sein, dass es gelungen ist, für den Patienten die geeignete Behandlung auszuwählen; andererseits ist damit noch nicht gesagt, wie erfolgreich eine bis zu Ende geführte Behandlung wirklich sein wird (Lindemeyer 2008). Aus der klinischen Erfahrung und langjähriger Weiterbegleitung von Alkoholabhängigen zeigt sich, dass selbst eine gute Compliance, eine hohe Zufriedenheit und klar formulierte Abstinenzmotivation im Rahmen der therapeutischen Maßnahme, langfristig keine sichere Stabilität hinsichtlich der Abstinenzsicherung ausweisen.

Der vermutlich sinnvollste Versuch, die Allokationsproblematik zu lösen, besteht nach den Ergebnissen der zitierten Klinikstudie (Lindemeyer 2008) also in der 
Bildung von Fallgruppen. Der einrichtungsunabhängige Behandlungsbedarf sollte die Zuordnung des Patienten in eine Fallgruppe bedingen und damit die Zuweisung in die geeignete Behandlungsstätte definieren. Prozessdaten aus der Basisdokumentation und dem KTL-System sowie Daten aus psychometrischen Assessments und Behandlungsergebnissen einer 1-Jahres-Katamnese wurden integriert.

Der Bedarf und die therapeutischen Leistungen wurden getrennt analysiert. Aus der Bedarfsebene ergaben sich drei Bedarfsklassen. Hierbei waren SCL-90-R, ICD 10, verschlüsselte Komorbidität, Therapiewiederholung, Arbeitslosigkeit und Abhängigkeitsdauer bedeutsam. Die Analyse der therapeutischen Leistungen machte deutlich, dass sich zwei unterschiedliche Bedarfsklassen ergeben. Allerdings zeigte sich auch, dass Unterschiede zwischen den Kliniken hinsichtlich des derzeit applizierten therapeutischen Programms vorliegen.

Welcher Faktor für die unterschiedliche Therapiezusammensetzung maßgeblich war, konnte nicht geklärt werden. Die Bedarfsfeststellung, beispielsweise bei Einzelgesprächen, erschien sehr subjektiv. Sie war vom Störungsbild des Patienten, als auch von der therapeutischen Vorgehensweise des Behandlers abhängig. Eine Mittelung oder Standardisierung erscheint in diesem Bereich zwar rein rechnerisch möglich, sie dürfte aber ein inadäquates Praxiselement darstellen. Bei der Indikation arbeitsbezogener Leistungen wurde deutlich, dass Effekte erst in der Häufung messbar auftraten. Entsprechend wurde hier der Bedarf kumulativ bestimmt. Er konnte prädiktorisch nur grob orientierend festgelegt werden.

In der erwähnten Klinikstudie (Lindemeyer 2008) war mit Hilfe aufwändiger statistischer Verfahren die Fallgruppenbildung möglich. Die drei dort unterschiedenen Patientengruppen wiesen nach einem Jahr auch signifikant unterschiedliche Katamneseergebnisse auf. 


\subsubsection{Schlussfolgerungen aus den Studienergebnissen}

Aus den obigen Studien, in denen die Ergebnisse der Rehabilitationsforschung in klinischer Anwendung überprüft wurden, wurden die nachstehenden Schlussfolgerungen gezogen (Lindenmeyer 2008).

- Patientenfallgruppen können die Zugangssteuerung durch den Rententräger erleichtern.

- Die Zuweisungsqualität erscheint durch Fallgruppen verbessert.

- Die Frage ambulant oder stationär kann auf eine rationale Grundlage gestellt werden.

- Kliniken müssen anhand ihrer Ausstattung bzw. ihrer therapeutischen Möglichkeiten für die verschiedene Fallgruppen als taugliche Einrichtungen beurteilt werden.

- Die vom Setting abhängigen Erfolgsaussichten müssen ins Verhältnis zu drohenden Gefährdungen, wie beispielsweise Rückfällen, gesetzt werden.

- Unterschiedliche Strukturqualitäten in Kliniken müssen nicht zwingend angepasst, sondern können vielmehr durch Fallgruppenzuordnung ausgeglichen und genutzt werden.

- Fallgruppen machen die Qualität einer Allokationsentscheidung innerhalb einer Einrichtung überprüfbar.

- Patientenklassifikationssysteme eignen sich nur bedingt zur individuellen Therapiegestaltung.

- Die Allokationsentscheidung muss noch immer individuell formuliert werden.

- Eine Reduktion des diagnostischen Aufwandes kann nicht erfolgen.

- Vergütungssätze können leichter berechnet werden.

\subsection{Untersuchung}

\subsubsection{Ort und strukturelle Umgebung der Untersuchung}

Die vorliegende Untersuchung wurde im Diakonie-Krankenhaus Harz GmbH (DKH), Brockenstraße 1 in 38875 Elbingerode durchgeführt. Das hier befindliche 
Suchtzentrum besteht aus einem Akut-Krankenhausbereich mit Innerer Medizin, Psychiatrie und psychotherapeutischer Medizin. Weiterhin befindet sich hier die Klinik zur medizinischen Rehabilitation für Suchtkranke mit 132 Plätzen zur stationären Langzeitentwöhnung. Angegliedert sind ein Ärztehaus und die psychiatrische Institutsambulanz. Im Umgebungsradius von ca. $15 \mathrm{~km}$ befindet sich der Komplementärbereich der Einrichtung. In inm sind die Adaption, das ambulant betreute Wohnen, die Tagesstätte, das sozialtherapeutische Wohnen und das Heim für chronisch mehrfach Geschädigte sowie die koordinierenden Suchtberatungsstellen angesiedelt.

Die zu Grunde gelegten Patientendaten stammen aus der Datensammlung der Rehabilitationsklinik, in der pro Jahr ca. 500 Patienten behandelt werden. Die Patientenklientel setzt sich aus Männern und Frauen zusammen. Die Zuweisungsund Behandlungsdiagnose ist die stoffliche Abhängigkeit von Alkohol, Medikamenten und Drogen, jeweils einzeln oder in Kombination. Psychische und körperliche Komorbiditäten mit unterschiedlichem Schweregrad werden regelhaft mitbehandelt.

Inhaltlich orientiert sich die Behandlung und Betreuung am biopsychosozialen Krankheitsmodell. Neben der Behandlung der Pathologika sind Ressourcenaufbau und Wiederherstellung von Fähigkeiten sowie die Ordnung der sozialen Verhältnisse und die Lebenssinnstiftung die Hauptansatzpunkte der rehabilitativtherapeutischen Arbeit. Das zugrundeliegende Wertesystem für den hier beschriebenen ganzheitlichen Therapieansatz bildet das christliche Krankenhausleitbild (siehe Anhang 1).

Der Träger der Einrichtung ist die Diakonie-Krankenhaus Harz GmbH. Der Gesellschafter ist der Deutsche Gemeinschafts-Diakonieverband e. V. (DGD).

\subsection{2 Überblick über die Methodik der Untersuchung}

Die Bearbeitung der beschriebenen Fragestellung erfolgte anhand der soziodemographischen Daten, den Befunden der testpsychologischen Untersuchungen, der Auswertung der Entlassberichte, der Basisdokumentation Sucht (für Einrichtungen des Fachverbandes Sucht sowie klinikintern) und der 1Jahres-Katamnese, jeweils bezogen auf Patienten des Entlassjahrgangs 2004 des Diakonie-Krankenhauses Harz GmbH in Elbingerode (DKH). 
Die Aufarbeitung der Zugangsdaten erfolgte zunächst in Form deskriptiver Statistik. Die Patientenstichprobe sollte damit in den für diese Arbeit interessierenden Merkmalsbereichen dargestellt werden.

Die aus den Haupthypothesen 1und 2 abgeleiteten spezifischen Hypothesen (siehe Punkt 1.1) wurden anhand des vorliegenden Datenmaterials statistisch überprüft. Die Haupthypothese 3 wurde nicht statistisch überprüft. Stattdessen wurden aus den vorliegenden Ergebnissen induktive Schlussfolgerungen abgeleitet.

Die gemessenen Outcomevariablen wurden ebenfalls zunächst deskriptiv erfasst. Um echte Effekte von zufälligen Befunden zu unterscheiden und um zu erkennen, in welchem Ausmaß sich Merkmale positiv oder negativ auf den Therapieerfolg auswirken, wurden die Ergebnisse dann statistisch überprüft und zwar jeweils in Bezug auf die interessierenden Zugangsvariablen bzw. die angenommenen Prädiktorvariablen. Dies geschah je nach dem Messniveau der Outcomevariablen per multipler linearer Regressionsanalyse bzw. per multipler logistischer Regressionsanalyse (Eid et al. 2010).

Die Daten bezüglich des Zugangswegs sowie alle anderen in die Untersuchung eingegangene Daten zum Betreuungsbeginn sind als Baseline für die Outcomevariablen des Betreuungsendes sowie der Katamneseerhebung, also der Ein-Jahres-Nachbefragung, zu sehen.

\subsubsection{Datengewinnung}

Im Verlauf einer medizinischen Rehabilitation fallen in den unterschiedlichsten Bereichen eine Fülle von Daten an. Diese sind sowohl verwaltungstechnisch, als auch für den diagnostischen Bereich und den Behandlungsprozess von Interesse. Zum Teil dienen sie auch der Datenerhebung der Fachverbände und finden Einsatz bei der Erstellung der jährlich erhobenen deutschen Suchthilfestatistik (Pfeiffer-Gerschel et al. 2010). Des weiteren sind alle Mitglieder des Fachverbundes Sucht e. V. (FVS), welchem auch die medizinische Rehabilitation des DKH angeschlossen ist, gehalten, nach einem Jahr eine Nachbefragung über die Nachhaltigkeit des Behandlungserfolges durchzuführen (die Ein-Jahres- 
Katamnese). Die in der Arbeit verwandten Daten wurden folglich nicht speziell für die vorliegende Untersuchung erhoben, sondern gezielt aus dem großen Fundus der vorhandenen Daten ausgewählt.

Im Folgenden werden zunächst die für die Untersuchung gewonnenen Daten unter Bezug auf die Datenquellen benannt.

\section{Stammdaten}

Hierunter fallen die soziodemographischen Daten wie "Alter" und "Geschlecht", aber auch „Aufnahmedatum und Entlassdatum", „Aufenthaltsdauer" sowie der „Kostenträger“. Diese Daten werden zum Teil standardmäßig mit Hilfe von „Patfak“, dem Rehabilitationspatientenverwaltungsprogramm, erfasst. Zum Teil werden sie aber auch durch die jeweiligen Bezugstherapeuten mit Hilfe des Patfak Programmmoduls zur Kerndatenerhebung Basisdokumentation Sucht der Deutschen Suchthilfestatistik (BADO) erhoben (siehe unten).

Eine Übersicht über alle erfassten Stammdaten befindet sich im Anhang 2.

\section{Entlassungsbericht}

Dieser gliedert sich in 2 Teile. Zum einen enthält er die standardisierten Formblätter 1, 1a und 1b mit Basisinformationen (z. B. Angaben über „Therapiedauer“, „Entlassform“, „Diagnosen“, „Arbeitsfähigkeit“), mit der sozialmedizinischen Beurteilung der Leistungsfähigkeit und mit der Dokumentation therapeutischer Leistungen. Zum Anderen umfasst er den Arztbericht mit Anamnese, Befunderhebung, Therapiezielen, Therapieverlauf und Rehabilitationsergebnis mit sozialmedizinischer Epikrise und Nachsorgeempfehlungen. In Form und Inhalt sind diese Berichte weitgehend standardisiert, die Formblätter ließen sich zum großen Teil quantitativ auswerten (Deutsche Rentenversicherung Bund 2009).

Die Auswertung der freiformulierten Therapeutendokumentationen und der Arztberichte wurde in folgender Herangehensweise durchgeführt.

Es wurden die Entlassberichte des Jahrgangs 2004 vollständig herangezogen. In diesen wurde von den Aufnahmetherapeuten ein Abschnitt „Ersteindruck und Einstiegsverhalten" formuliert. Diese Erstbeurteilung wurde von den Therapeuten 
aufgrund der Aussagen der Patienten im Erstgespräch und dem beobachteten Einstiegsverhalten in den ersten einführenden Veranstaltungen getätigt. Die Patienten wurden also ganz zu Beginn der Therapie hinsichtlich ihrer Abstinenzmotivation, der Therapiemotivation und ihrer Veränderungsmotivation eingeschätzt. Es ergaben sich hieraus drei unterscheidbare Gruppen, um der Frage nach der Eigenmotivation der Patienten nachzugehen:

1) Die explizit und glaubwürdig eine „eigene Motivation“ formuliert haben sowie die Patienten, die "gut motiviert" wirkten.

2) Die „Fremdmotivierten“, die ausdrücklich angaben, keine Rehabilitationsmaßnahme zu wollen, d. h. die aufgrund einer Auflage die Maßnahme zu absolvieren hatten.

3) Die „ambivalent motivierten Patienten“, die entweder aussagten, noch unentschieden zu sein, was ihr Therapieergebnis erbringen sollte, oder aber die wohl mitteilten, motiviert zu sein, die aber in ihrem Verhalten keinen eigenen Ansatz bezüglich Abstinenz, Therapie und Veränderung zeigten.

\section{Basisdokumentation Sucht zur Kerndatenerhebung der Deutschen Suchthilfestatistik}

„BADO“ ist in fünf große Abschnitte gegliedert: Sie enthält die Stammdaten des Patienten (siehe oben), Fragen zum Betreuungsbeginn, Fragen, die sowohl bei Betreuungsbeginn als auch bei Betreuungsende beantwortet werden, Fragen zum Betreuungsende sowie Angaben zu den Diagnosen zum Betreuungsbeginn. Die gesamten Items der Kerndatenerhebung befinden sich im Anhang 3.

\section{Testpsychologische Untersuchungen}

In der medizinischen Rehabilitation Sucht des DKH findet seit einigen Jahren eine umfassende, computergestützte testpsychologische Untersuchung in der Aufnahmewoche statt.

Diese gliedert sich in zwei Bereiche auf:

1) Das neuropsychologische Screening:

Es dient der Abschätzung des (prämorbiden) kognitiven Leistungsniveaus in Verbindung mit der Erfassung der aktuellen kognitiven Leistungsfähigkeit. Das Ergebnis offenbart, ob von einer erworbenen cerebralen Insuffizienz ausgegangen werden muss. Genutzt werden hier 
der Wortschatztest (WST) (Schmidt und Metzler 1992), die c.l.-Skala (Weidenhammer und Fischer 1987), ein Selbstbeurteilungsverfahren, das die klinische Symptomatik einer beginnenden cerebralen Insuffizienz erfasst, sowie der Verbalgedächtnis-Test (VEG) (Hänsgen und Rosenfeldt 2005) zur Diagnostik gestörter verbaler Merkfähigkeit, der insbesondere im unteren Leistungsbereich differenziert und auch Aufmerksamkeitsdefizite erfasst.

2) Persönlichkeitsdiagnostik:

Hier wird regelhaft das Persönlichkeits-Stil-und-Störungs-Inventar (PSSI) (Kuhl und Kazén 1997) dargeboten, laut Testhandbuch ein Selbstbeurteilungsinstrument, das die relative Ausprägung von Persönlichkeitsstilen quantifiziert. Diese gelten als nicht-pathologische Entsprechungen der in DSM-IV und ICD-10 beschriebenen Persönlichkeitsstörungen.

Bei der vorliegenden Untersuchung wurden aus Gründen der Variablenbegrenzung lediglich spezielle Skalen der jeweils durchgeführten Testverfahren ausgewählt:

- Der Wortschatztest (WST) zur Abschätzung des prämorbiden Leistungsniveaus.

- Aus dem Verbal-Gedächtnistest (VEG) die Skala 4, welche die verbale Merkfähigkeit des Langzeitgedächtnisses erfasst.

- Die c.l.-Skala, die die subjektiv wahrgenommene Anzahl erworbener kognitiver Beeinträchtigungen beschreibt.

- Vom Persönlichkeits-Stil-und-Störungs-Inventar (PSSI) die Skala 4 „selbstkritisch-selbstunsicher", um den Aspekt der sozialen Kompetenz abzuschätzen.

- Ebenfalls vom PSSI die Skala 11 „spontan-borderline“, um den Aspekt der Emotionalität bzw. der emotionalen Instabilität zu erfassen.

Mit diesen ausgewählten Skalen erschienen die Hauptkategorien der kognitiven Ressourcen sowie deren Defizite und die maßgeblichen Aspekte der Emotionalität, die sich auf den therapeutischen Prozess auswirken, erfasst. Die 
Möglichkeiten zur Kontinuitätsleistung, welche sowohl für den therapeutischen Prozess als auch für die Weiterentwicklung im posttherapeutischen Zeitraum, d. h. zunächst in der 1-Jahres-Entwicklung, die maßgebliche Rolle spielen, sind hier abgebildet. Auf die Übernahme weiterer testpsychologischer Befunde wurde verzichtet, da ansonsten die Übersichtlichkeit reduziert worden wäre. Dies hätte die Überfrachtung der Regressionsmodelle bedingt und das, obwohl insgesamt keine nennenswerte Zusatzinformation im Variablenbereich zu erwarten gewesen wäre.

\section{Katamnese-Fragebogen}

Wie bereits erwähnt wird vom Fachverband Sucht e. V., dem die Rehabilitationsklinik des DKH angegliedert ist, eine jährliche Katamnese in Form einer Vollbefragung erwartet. Diese dient der Qualitätssicherung der dem Verband angegliederten Suchthilfeangebote. Ihr wird ein „besonderer Wert [...] auf die Ergebnisqualität" (Missel et al. 2011, Seite 15) zugeschrieben.

Erfragt werden u. a. der Erwerbsstatus, der Familienstand, das Aufsuchen von Suchthilfe- und Nachsorgeeinrichtungen sowie, als besonders wichtiges Merkmal, die Abstinenz nach einem Jahr. Gibt ein Befragter an, rückfällig geworden zu sein, wird danach gefragt, seit welcher Zeit wieder Abstinenz vorliegt. Des Weiteren werden Rückfallursachen sowie der Grad der erreichten Zufriedenheit und der Veränderungen in verschiedenen Lebensbereichen abgefragt.

Der hierfür verwandte Katamnesebogen befindet sich in Anhang 4.

In der vorliegenden Arbeit wurden die 1-Jahres-Katamnese-Daten des Entlassjahrganges 2004 mit dem Nachbefragungsbogen zur stationären Entwöhnungsbehandlung (Fachausschuss Sucht des AHG Wissenschaftsrates 2002) herangezogen. Die Form der Katamneseerhebung orientierte sich dabei ebenfalls an den Programmvorgaben des Patfak Programmmoduls zur Kerndatenerhebung. Durchführung und Auswertung erfolgten angelehnt an die Standards zur Durchführung von Katamnesen bei Abhängigen (Deutsche Gesellschaft für Suchtforschung und Suchttherapie e.V. 2001). Alle Patienten wurden 1 Jahr nach ihrer Entlassung aus der Suchtrehabilitationseinrichtung des DKH mit der Bitte angeschrieben, einen mitgeschickten Katamnesebogen auszufüllen. Kamen die Patienten der Bitte beim ersten Anschreiben nicht nach, erfolgte noch ein zweites Anschreiben. 


\subsubsection{Durchführung der Untersuchung}

Die Daten von allen alkoholabhängigen Patienten des betreffenden Entlassjahrganges 2004 wurden erhoben.

Die Datensammlung sowie die Datenauswertung wurden von D. Kemmann, dem leitenden Psychologen des DKH, durchgeführt. Dieser ist in der Einrichtung seit Jahren für die Durchführung der katamnestischen Untersuchungen zuständig. Die gesamte statistische Auswertung erfolgte mit Hilfe des Statistik-Programms SPSS. Die Aufbereitung in tabellarischer Form erfolgte mit Hilfe des Tabellenkalkulationsprogramms MS Excel.

\section{Statistische Verfahren}

Neben der rein deskriptiven Statistik, welche die Vorkommens-Häufigkeit bestimmter Merkmale beschreibt, wurde zur empirischen Überprüfung der spezifischen Hypothesen das Verfahren der multiplen linearen bzw. der multiplen logistischen Regression verwandt. Diese sollen an dieser Stelle kurz erläutert werden. Dabei wird auf das Lehrbuch „Statistik und Forschungsmethoden“ (Eid et al. 2010) zurückgegriffen.

\section{Multiple lineare Regression}

Die multiple lineare Regression ist eine Erweiterung der einfachen linearen Regression, bei der Unterschiede in einer abhängigen Variablen auf Unterschiede nicht nur in einer, sondern in mehreren unabhängigen Variablen, zurückgeführt werden. Die multiple Regression beschreibt dabei einen "gerichteten Zusammenhang“. „Diese Gerichtetheit ist die Grundlage für weitere Zielsetzungen der multiplen Regressionsanalyse: Prognose (Prädiktion) und Erklärung." (Eid et al., Seite 602). Dies entspricht dem Ziel der vorliegenden Arbeit, nämlich Prädiktorvariablen zu erkennen, die möglichst „viel“ Vorhersagekraft für das untersuchte Outcome haben.

Voraussetzung für die Durchführung einer multiplen linearen Regression ist, dass die abhängige Variable metrischer Natur ist. Allerdings bietet die multiple lineare Regression den Vorteil, dass mit inr unabhängige Variablen verschiedener Skalenniveaus analysiert werden können. Dies genau liegt bei dieser Arbeit vor: So gibt es kategoriale Variablen wie den „Erwerbsstatus“ (z. B. „erwerbstätig“, 
„erwerbslos“), aber auch metrische Variablen wie die Dauer der Abhängigkeit, die in Jahren angegeben wird.

\section{Exkurs „Metrische und Dummy-Variablen“}

An dieser Stelle sei gesagt, dass die metrischen Variablen unverändert in die Analyse eingehen können, aus den kategorialen Variablen aber so genannte „Dummy-Variablen“ gebildet werden müssen. Dabei wird die Ausprägung des Merkmals mit „1“ kodiert, das Nichtvorhandensein des Merkmals mit „0“. Die Dummy-Variablen werden dann zur so genannten „Referenzkategorie“ der jeweiligen Variable ins Verhältnis gesetzt. Ein Beispiel im Vorgriff auf die vorliegenden Ergebnisse der Arbeit soll das verdeutlichen:

Der Einfluss auf das „Therapieergebnis“ (entspricht einer abhängigen Variablen) sollte überprüft werden. Eine der in die Untersuchung eingegangene unabhängige Variable ist die „Motivationslage“, die sich aus den drei Kategorien „eigenmotiviert“, „ambivalent motiviert“ und „fremdmotiviert“ zusammensetzt. „Eigenmotiviert“ wurde dabei als Referenzkategorie definiert. In den Ergebnissen zeigt sich, dass sich die Kategorie "fremdmotiviert" signifikant auf das Therapieergebnis auswirkt und zwar negativ. Diese Aussage ist im Verhältnis zur Kategorie „eigenmotiviert“ zu treffen. Die Referenzkategorie taucht in der Ergebnisdarstellung selbst gar nicht auf, es wird aber bei der Darstellung der unabhängigen Variablen jeweils erläutert, welches die entsprechende Referenzkategorie ist.

Bei der Auswertung der metrischen unabhängigen Variablen gilt folgende Regel: Ist der Effekt signifikant, führt die Erhöhung der unabhängigen Variablen zu einer bedeutsamen Erhöhung (positiver Effekt) bzw. Verringerung (negativer Effekt) der abhängigen Variablen. Denn: Die „multiple Regressionsanalyse [...] ermöglicht es [...], den Einfluss einer unabhängigen auf die abhängige Variable zu bestimmen, wenn der Einfluss weiterer unabhängiger Variablen kontrolliert wird." (Eid et al. 2010, Seite 777)

Die Signifikanzniveaus werden wie üblich ab einer Sicherheitswahrscheinlichkeit (p) von $\mathrm{p}<0,05$ ausgewiesen.

Für die vorliegende Arbeit sind zwei Aspekte bei der Auswertung der multiplen linearen Regression von Interesse:

- Zum Einen das Signifikanzniveau des Gesamtmodells: Leisten die unabhängigen Variablen als Gesamtmodell einen Beitrag zur Erklärung 
von Unterschieden bei der abhängigen Variablen? Dabei ist auch der Anteil ,aufgeklärter Varianz“ von Interesse, d. h. wie hoch die Varianz der abhängigen Variablen ist, die durch die Varianz der unabhängigen Variablen erklärt wird.

- Zum Zweiten interessiert die Frage, welche Variablen genau bzw. welche Unterkategorien sich signifikant, also bedeutsam, auf die Varianz der abhängigen Variablen auswirken? Diese beiden Fragen sowie die Richtung des Effektes der jeweiligen Variable wurden bei der Ergebnisdarstellung berücksichtigt.

\section{Multiple logistische Regression}

Ein Problem dieser Arbeit ist, dass nicht alle Outcomevariablen metrischer Natur sind. Bei dichotomen abhängigen Variablen wie beispielsweise "Abstinenz" (nämlich Ja/Nein) kann nicht auf die lineare Regression zurückgegriffen werden, die besagt, wie hoch die "Vorhersagekraft" der Variation eines Bündels unabhängiger Variablen auf die Varianz der abhängigen Variablen ist. Stattdessen kann nur die "Wahrscheinlichkeit“ angegeben werden, „[...] ein bestimmtes Verhalten zu zeigen. [...] Diese Wahrscheinlichkeit kann mit der Zunahme der Werte der unabhängigen Variablen ansteigen (positiver Zusammenhang), abfallen (negativer Zusammenhang) oder gleich bleiben (Unabhängigkeit)“. (Eid et al. 2010, Seite 767)

Auch hier besteht die Möglichkeit, nicht nur metrische, sondern auch kategoriale Variablen in die Analyse mit einzubeziehen (ebenfalls durch das Bilden von Dummy-Variablen), sodass der Effekt der selben unabhängigen Variablen untersucht werden kann, wie bei der linearen Regression. Der Unterschied (und Vorteil) besteht hier nur darin, dass bei der Bildung der Dummy-Variablen der Effekt der gesamten kategorialen unabhängigen Variable mit in die Berechnung eingeht. Bei der „Motivationslage“ sieht man also nicht nur den möglichen Effekt der Unterkategorien „fremdmotiviert“ und „ambivalent motiviert" im Vergleich zu "eigenmotiviert" auf das Outcome, sondern auch, ob die gesamte Variable „Motivationslage“ sich signifikant auf die Wahrscheinlichkeit auswirkt, d. h. ob es zu einem bestimmten Outcome kommt.

Von Interesse für die vorliegende Arbeit sind folgende Maße, auf die wiederum bei der Ergebnisdarstellung eingegangen wird: 
- In der jeweils dargestellten Modellzusammenfassung findet sich das Maß für die Effektstärke. Die Stärke des Zusammenhangs der abhängigen Variablen mit den unabhängigen Variablen wird hier annäherungsweise in Form von Nagelkerkes R-Quadrat beschrieben (Eid et al. 2010). Nagelkerkes R-Quadrat ist ein Maß für die aufgeklärte gemeinsame Varianz. Daraus lässt sich auch ableiten, wie hoch die Vorhersagekraft der zusammengefassten unabhängigen Variablen insgesamt auf die OutcomeVariable ist.

- Des Weiteren ist die Klassifizierungsgüte von Interesse: Wie hoch ist die Übereinstimmung des vorhergesagten Outcomes durch die im Modell zusammengefassten unabhängigen Variablen mit dem tatsächlich vorgefundenem Outcome?

- Zeigt sich ein statistisch signifikanter Einfluss der im Modell erfassten einzelnen unabhängigen Variablen auf das jeweilige Outcome? Wenn ja, wie ist die Richtung des Einflusses?

\subsubsection{Darstellung der Untersuchungsstichprobe}

Von 521 im Jahr 2004 entlassenen Patienten wurden 434 mit der Erstdiagnose Alkoholabhängigkeit (ICD-10: F10.2) behandelt.

Tabelle 1 (Stichprobenbeschreibung hinsichtlich der untersuchten Zugangsmerkmale) stellt weitgehend die Merkmale dar, die in der weiteren empirischen Untersuchung eine wesentliche Rolle spielen. Die Daten entstammen der Stammdatenerfassung des Rehabilitationsdokumentations-programms „Patfak“, den Rehabilitationsentlassberichten sowie der „BADO“ (siehe 2.4.3). Im Unterpunkt „Vermittlung“ werden die Zugangswege dargestellt. Sie sind untergliedert nach Zugangswegen von Patienten ohne Auflage $(N=269)$ sowie von Patienten mit Auflage $(N=102)$. „Vermittlung“ meint hier den primären bzw. den entscheidenden Anstoß zur Aufnahme einer Therapie. Die Gruppe „mit Auflage" umfasst die Patienten, die über den „Arbeitgeber“, eine „Gerichtsauflage“, einen "gerichtlich bestellten Betreuer" sowie einer Auflage seitens des „Rententrägers“, des „Arbeitsamtes“ oder aber einer „anderen Behörde“ zur Therapie kamen. "Ohne Auflage" kamen demnach Patienten, die über die „Suchtberatung“, über die „Selbsthilfegruppe“, über den „Hausarzt“, direkt über die 
Klinik, die die Entgiftungsbehandlung durchführte („ohne Nahtlosverlegung“ und „mit Nahtlosverlegung“) sowie „ohne Angaben und gleichzeitig ohne Auflagen“ (wo zwar der Aspekt der Auflage, nicht aber der des Vermittlungsweges eruiert werden konnte). ( $(\mathrm{n}=63)$ Patienten waren diesbezüglich ohne Angaben.

Der für die spezifische Hypothese 1 interessierende Punkt der Motivationslage wurde bereits genauer erläutert. Alle anderen Merkmalsbereiche stellen mögliche Prädiktoren dar, die einen Einfluss auf das Outcome haben könnten, welche in der spezifischen Hypothese 2 überprüft werden. Die meisten Variablen (,Geschlecht“, „Alter“, „Familienstand“, „Schulabschluss“, „ausgewählte Testskalen, vgl. 2.4.3“, "Abhängigkeitsdauer", „zuvor suchtbezogene Hilfe bekommen" sowie „Wiederaufnahme in der Klinik") erklären sich von selbst.

Der Bereich der "Erwerbstätigkeit“ wird hier noch etwas differenzierter dargestellt, als in der späteren Untersuchung zusammengefasst: Neben „selbstständiger Berufstätigkeit“ und "nicht selbstständiger Berufstätigkeit“" werden später auch die Eigenschaften „Schüler" sowie „Hausfrau/-mann“ unter „erwerbstätig“ gefasst, da eine fest definierte Beschäftigung besteht. Daraus ergeben sich dann $(N=139)$ Erwerbstätige. Dass im Merkmalsbereich „Dauer Arbeitslosigkeit unmittelbar vor der Rehabilitation“ die Gruppe der noch Berufstätigen mit ( $\mathrm{N}=99)$ kleiner ist als die beschriebene Gruppe der Erwerbstätigen, liegt an der unvollständigen Datenlage der Basisdokumentation vgl. Kategorie "ohne Angaben“ $(\mathrm{N}=100)$. Im Bereich der "Komorbidität" wird nur das Vorhandensein der Zweit- und Folgediagnosen erfasst, nicht jedoch inre Anzahl. Sicherlich geht dadurch Information verloren, dennoch soll dieser Aspekt hier genügen, um den Einfluss des Vorhandenseins komorbider Erkrankungen auf das Outcome zu erfassen. Bei den Leistungsträgern werden noch die alten Bezeichnungen für die regionalen Rentenversicherungen übernommen, da im Jahr 2004, d. h. zum Zeitpunkt der Datenerhebung, noch nicht die gemeinsame Bezeichnung DRV eingeführt war. Die regionalen Rentenversicherungen, außer derer von Sachsen-Anhalt und Thüringen, wurden zusammengefasst, ebenso alle Krankenkassen und die Versicherten der jetzt zusammengenommenen Rentenversicherung Knappschaft Bahn-See, da sonst die Gruppengrößen zu klein geworden wären. 
Tabelle 1: Stichprobenbeschreibung der untersuchten Zugangsmerkmale von behandelten Alkoholabhängigen, Jahrgang 2004, des Diakoniekrankenhaus Harz $\mathrm{GmbH}$. Wenn nicht anders angegeben, Angaben der Häufigkeit in Absolutzahlen (N) und Prozent (\%), sonst Variablenbeschreibung mit Mittelwert und Standardabweichung

Legende:

$$
\begin{aligned}
& \text { Grau hinterlegt ist die Tabellenüberschrift } \\
& \text { WST = Wortschatztest; die Werte beziehen sich auf den IQ = Intelligenzquotienten } \\
& \text { c.I.-Skala = Skala zu Erfassung cerebraler Insuffizienzen } \\
& \text { C- und T-Norm-Werte = geben Auskunft über Verhältnis von Testwert zu Mittelwert und } \\
& \text { Standardabweichung der Normstichprobe eines Tests } \\
& \text { PSSI = Persönlichkeits-Stil-und-Störungs-Inventar } \\
& \text { POS = Polytechnische Oberschule }
\end{aligned}
$$

\begin{tabular}{|c|c|c|c|}
\hline Merkmal & Kategorie & $\begin{array}{l}\text { Gesamt } \\
\text { N= } 434 \\
\text { Anzahl }\end{array}$ & $\begin{array}{l}\text { chprobe } \\
\%\end{array}$ \\
\hline Geschlecht & $\begin{array}{l}\text { Männlich } \\
\text { Weiblich }\end{array}$ & $\begin{array}{l}349 \\
85\end{array}$ & $\begin{array}{l}80,4 \\
19,6\end{array}$ \\
\hline Altersgruppe & $\begin{array}{l}\text { Bis } 30 \text { Jahre } \\
\text { Über } 30 \text { bis } 40 \text { Jahre } \\
\text { Über } 40 \text { bis } 50 \text { Jahre } \\
\text { Über } 50 \text { bis } 60 \text { Jahre } \\
\text { Über } 60 \text { Jahre }\end{array}$ & $\begin{array}{l}19 \\
144 \\
184 \\
76 \\
11\end{array}$ & $\begin{array}{l}4,4 \\
33,1 \\
42,4 \\
17,6 \\
2,6\end{array}$ \\
\hline $\begin{array}{l}\text { Familienstand bei } \\
\text { Aufnahme }\end{array}$ & $\begin{array}{l}\text { ledig } \\
\text { Verheiratet, zusammenlebend } \\
\text { Verheiratet, getrenntlebend } \\
\text { Geschieden } \\
\text { Verwitwet } \\
\text { Ohne Angaben }\end{array}$ & $\begin{array}{l}125 \\
137 \\
32 \\
130 \\
5 \\
5\end{array}$ & $\begin{array}{l}28,8 \\
31,6 \\
7,4 \\
30 \\
1,2 \\
1,2 \\
\end{array}$ \\
\hline Höchster Schulabschluss & $\begin{array}{l}\text { Kein Schulabschluss } \\
\text { Sonderschulabschluss } \\
\text { Hauptschulabschluss/ POS 8. Klasse } \\
\text { Realschulabschluss/ POS 10. Klasse } \\
\text { (Fach-)Hochschulreife } \\
\text { Andere } \\
\text { Ohne Angaben }\end{array}$ & $\begin{array}{l}15 \\
16 \\
102 \\
243 \\
46 \\
8 \\
4\end{array}$ & $\begin{array}{l}3,5 \\
3,7 \\
23,5 \\
56,0 \\
10,6 \\
1,8 \\
0,9\end{array}$ \\
\hline $\begin{array}{l}\text { Erwerbstätigkeit bei } \\
\text { Aufnahme }\end{array}$ & $\begin{array}{l}\text { Erwerbstätig } \\
\text { Erwerbslos } \\
\text { Schüler/Student } \\
\text { Berentet } \\
\text { Sonstiges (Hausfrau/-mann) } \\
\text { Ohne Angaben }\end{array}$ & $\begin{array}{l}134 \\
248 \\
1 \\
33 \\
4 \\
14\end{array}$ & $\begin{array}{l}30,9 \\
57,1 \\
0,2 \\
7,6 \\
0,9 \\
3,2\end{array}$ \\
\hline $\begin{array}{l}\text { Wortschatztest (WST) (IQ- } \\
\text { Werte) }\end{array}$ & (Mittelwert, Standardabweichung) & 95,99 & 12,21 \\
\hline $\begin{array}{l}\text { Verbalgedächtnistest } \\
\text { (VEG) - Skala } 4 \text { (C-Norm- } \\
\text { Werte) }\end{array}$ & (Mittelwert, Standardabweichung) & 3,87 & 2,20 \\
\hline c-I.-Skala (Rohwerte) & (Mittelwert, Standardabweichung) & 15,22 & 9,03 \\
\hline $\begin{array}{l}\text { PSSI - ,selbstkritisch - } \\
\text { selbstunsicher“ (T-Norm- } \\
\text { Werte) }\end{array}$ & (Mittelwert, Standardabweichung) & 55,05 & 8,84 \\
\hline
\end{tabular}


Fortsetzung Tabelle 1: Stichprobenbeschreibung der untersuchten Zugangsmerkmale von behandelten Alkoholabhängigen, Jahrgang 2004, des Diakoniekrankenhaus Harz $\mathrm{GmbH}$. Wenn nicht anders angegeben, Angaben in Absolutzahlen (N) und Prozent (\%), sonst mit Mittelwert und Standardabweichung

\begin{tabular}{|c|c|c|c|}
\hline Merkmal & Kategorie & $\begin{array}{l}\text { Gesam } \\
N=434 \\
\text { Anzahl }\end{array}$ & $\begin{array}{l}\text { chprobe } \\
\%\end{array}$ \\
\hline $\begin{array}{l}\text { PSSI - ,,spontan - border- } \\
\text { line“" (T-Norm-Werte) }\end{array}$ & (Mittelwert, Standardabweichung) & 53,21 & 8,79 \\
\hline Motivationslage & $\begin{array}{l}\text { Eigenmotiviert } \\
\text { Ambivalent motiviert } \\
\text { Fremdmotiviert } \\
\text { Ohne Angaben }\end{array}$ & $\begin{array}{l}294 \\
83 \\
56 \\
1\end{array}$ & $\begin{array}{l}67,7 \\
19,1 \\
12,9 \\
0,2\end{array}$ \\
\hline \multirow[t]{3}{*}{\begin{tabular}{|l|} 
Vermittlung/ \\
Zugangsweg
\end{tabular}} & $\begin{array}{l}\text { Ohne Angaben, dabei ohne Auflagen } \\
\text { Suchtberatung } \\
\text { Selbsthilfegruppe } \\
\text { Hausarzt } \\
\text { Entgiftung } \\
\text { Entgiftung mit Nahtlosverlegung } \\
\text { Gesamt „ohne Auflage“ }\end{array}$ & $\begin{array}{l}18 \\
135 \\
11 \\
25 \\
57 \\
23 \\
269\end{array}$ & $\begin{array}{l}4,1 \\
31,1 \\
2,5 \\
5,8 \\
13,1 \\
5,3 \\
61,9\end{array}$ \\
\hline & $\begin{array}{l}\text { Arbeitgeber } \\
\text { Gerichtlicher Betreuer } \\
\text { Gericht } \\
\text { Auflage Rentenversicherung } \\
\text { Auflage Arbeitsamt } \\
\text { Auflage andere Behörden } \\
\text { Gesamt „mit Auflage“ }\end{array}$ & $\begin{array}{l}14 \\
7 \\
13 \\
20 \\
34 \\
14 \\
102\end{array}$ & $\begin{array}{l}3,2 \\
1,6 \\
3 \\
4,6 \\
7,8 \\
3,2 \\
23,4\end{array}$ \\
\hline & Ohne Angaben & 63 & 14,5 \\
\hline Leistungsträger & $\begin{array}{l}\text { LVA Sachsen-Anhalt } \\
\text { LVA Thüringen } \\
\text { andere LVAs } \\
\text { BfA } \\
\text { Krankenkassen } \\
\text { Knappschaften/Heilfürsorge/ } \\
\text { Bahnversicherung } \\
\text { Andere }\end{array}$ & $\begin{array}{l}225 \\
18 \\
14 \\
93 \\
29 \\
44 \\
11\end{array}$ & $\begin{array}{l}51,8 \\
4,2 \\
3,1 \\
21,4 \\
6,4 \\
10,1 \\
2,4\end{array}$ \\
\hline \begin{tabular}{|l|} 
Abhängigkeitsdauer \\
Jahren $(\mathrm{N}=338)$
\end{tabular} & (Mittelwert, Standardabweichung) & 12,78 & 9,34 \\
\hline 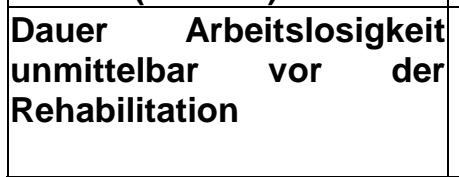 & $\begin{array}{l}0 \text { Monate } \\
1-12 \text { Monate } \\
>12 \text { Monate } \\
\text { Ohne Angaben }\end{array}$ & $\begin{array}{l}99 \\
72 \\
163 \\
100\end{array}$ & $\begin{array}{l}22,8 \\
16,6 \\
37,5 \\
23\end{array}$ \\
\hline $\begin{array}{l}\text { Zuvor suchtbezogene } \\
\text { Hilfe bekommen }\end{array}$ & $\begin{array}{l}\text { Nein } \\
\text { Ja } \\
\text { Keine Angaben }\end{array}$ & $\begin{array}{l}46 \\
383 \\
5 \\
\end{array}$ & $\begin{array}{l}10,6 \\
88,2 \\
1,2\end{array}$ \\
\hline $\begin{array}{l}\text { Wiederaufnahme in der } \\
\text { Klinik }\end{array}$ & $\begin{array}{l}\text { Nein } \\
\text { Ja } \\
\text { Keine Angaben }\end{array}$ & $\begin{array}{l}301 \\
127 \\
6\end{array}$ & $\begin{array}{l}69,4 \\
29,3 \\
1,4\end{array}$ \\
\hline $\begin{array}{l}\text { Komorbidität } \\
\text { (dargestellt wird das } \\
\text { Vorhandensein von } \\
\text { Zusatzerkrankungen) }\end{array}$ & $\begin{array}{l}\text { Körperbezogene Komorbidität } \\
\text { Psychische Erkrankungen } \\
\text { Alkoholbezogene Erkrankungen } \\
\text { Zweite Suchtdiagnose } \\
\text { (außer Tabakabhängigkeit) } \\
\text { Tabakabhängigkeit } \\
\text { Persönlichkeitsstörung } \\
\text { Intelligenzminderung }\end{array}$ & $\begin{array}{l}394 \\
253 \\
38 \\
\\
29 \\
275 \\
241 \\
9\end{array}$ & $\begin{array}{l}90,7 \\
58,3 \\
8,8 \\
6,7 \\
63,4 \\
55,5 \\
2,1\end{array}$ \\
\hline
\end{tabular}


Die Datenqualität ist weitgehend als hoch zu bewerten, da die Rate der MissingWerte („ohne Angaben“) in der Regel deutlich unter $5 \%$ bleibt. Hierbei gibt es zwei deutliche Ausnahmen: die "Dauer der Arbeitslosigkeit“ weist eine hohe Anzahl fehlender Angaben von $23 \%(N=100)$ auf. Der "Zugangsweg“, der für diese Arbeit maßgeblich interessierte, weist ebenfalls einen höheren Anteil fehlender Angaben von 14,5\% $(N=63)$ auf. Dies führt in einem Teil der empirischen Überprüfung der spezifischen Hypothesen zu einer kleineren Anzahl von Fällen, die in die Untersuchung eingingen. Es ist aus unserer Erfahrung bezüglich der hausinternen Basisdokumentation allerdings nicht davon auszugehen, dass es sich um einen systematischen Fehler handelt; alle Zugangswege dürften gleichermaßen von dieser lückenhaften Dokumentation betroffen sein.

$\mathrm{Da}$ mehrere unabhängige Variablen nominalskaliert waren, mussten daraus Dummy-Variablen gebildet werden (Eid et al. 2010). Die jeweilige Referenzkategorie innerhalb einer unabhängigen Variablen - also diejenige Kategorie, in Bezug zu der eine Aussage über den Effekt der „Dummyunabhängigen-Variablen“ auf die abhängige Variable getroffen wird - ist im Folgenden für alle durchgeführten Regressionen aufgelistet:

- „Auflage“: Referenzkategorie ist „keine Auflage“

- "Motivationslage“: Referenzkategorie ist „Eigenmotivation“

- "Geschlecht“: Referenzkategorie ist „männlich“

- "Dauer Arbeitslosigkeit“: Referenzkategorie ist "Arbeitslosigkeit $<1$ Monat“. Hier muss noch hinzugefügt werden, dass die 8 dargestellten Kategorien auf 3 Kategorien reduziert wurden, und zwar analog der heute üblichen Einteilung der „Erwerbstätigen“, der „Kurzzeit-Arbeitslosen“ $<1$ Jahr (wären in der Regel heute Arbeitslosengeld I - Empfänger) sowie der "LangzeitArbeitslosen" $>1$ Jahr (wären in der Regel heute Arbeitslosengeld II - Empfänger).

- „Erwerbssituation (Anfang)“: Referenzkategorie ist „erwerbstätig“

- „Familienstand“: Referenzkategorie ist „Verheiratet - zusammenlebend“

- "Schule“: Referenzkategorie ist „Abitur/(Fach)Hochschulreife“

- "Leistungsträger“: Referenzkategorie ist „BfA“ (Bundesversicherungsanstalt für Angestellte - heutige DRV Bund) 
- „Wiederaufnahme“: Referenzkategorie ist „nein“ (also „keine Wiederaufnahme“)

- „zuvor suchtbezogene Hilfe“: Referenzkategorie ist „nein“ (also „zuvor keine suchtbezogene Hilfe erhalten").

Um den Einfluss aller untersuchten unabhängigen Variablen auf die jeweils interessierende abhängige Variable genauer zu erfassen (diese ist für die spezifischen Hypothesen 1 und 2 identisch), wurden sie für beide spezifischen Hypothesen zusammengefasst. So lässt sich im Nachhinein auch zuverlässiger sagen, ob sich die Zugangswege oder doch mehr die zugangsrelevanten Variablen auf die abhängigen Variablen auswirken.

\subsubsection{Darstellung der Outcome-Variablen}

Primär interessierende Outcome-Kriterien sollen sein:

- „Entlassform“; dabei wurden die Entlassformen „reguläre Entlassung“ sowie „Entlassung mit ärztlich-/therapeutischem Einverständnis" als reguläre Entlassformen gewertet und mit „1“ kodiert. Die Entlassformen „Entlassung auf ärztlich-/therapeutische Veranlassung“, „Entlassung gegen ärztlich/therapeutischen Rat“, „disziplinarische Entlassung“ sowie „Entlassung durch Verlegung in andere Einrichtung" wurden als irreguläre Entlassformen gewertet und mit „0“ kodiert. Der Einfluss auf diese binäre Outcomevariable wird per logistische Regression gerechnet.

- „Therapieergebnis“; dieses ist vom Bezugstherapeuten des Patienten nicht nur in Textform im Entlassungsbericht beschrieben, sondern auch in der „BADO“ in Form eines Ratings abgegeben worden. „1= erfolgreich“, „2 = gebessert", „3 = unverändert" sowie „4 = verschlechtert" sind hier die möglichen Einschätzungen. Auch hier liegt ein Ordinalskalenniveau vor. Die Daten werden also per linearer Regression gerechnet.

- „Leistungsbild bei Entlassung“; dieses wird vom Arzt zum Einen bezogen auf die letzte berufliche Tätigkeit, zum Anderen bezogen auf den allgemeinen Arbeitsmarkt eingeschätzt. Für die vorliegende Arbeit wurde auf die allgemeinere Kategorie "Leistungsbild für den allgemeinen Arbeitsmarkt" zurückgegriffen. Unterschieden wird auf folgende Weise: „unter 3 Stunden arbeitsfähig“, „zwischen 3 und 6 Stunden arbeitsfähig“, sowie, „mehr als 6 Stunden arbeitsfähig“. Hier ist von einem (metrischen) Ordinalskalenniveau 
auszugehen, entsprechend wird bei der Auswertung die lineare Regression gerechnet.

- „Abstinenzrate nach einem Jahr“; diese wurde über das Katamneseanschreiben erfasst. Jede angegebene Form des Rückfalls wird später in der Untersuchung als Rückfälligkeit gewertet, auch wenn wieder eine Abstinenzzeit länger als 12 Wochen zum Zeitpunkt der Befragung bestand. Die Abstinenzrate wird als Dummy-Variable kodiert, wobei „,“" als rückfällig gilt und „1“ als abstinent. Hier wird der Einfluss auf die binäre Outcomevariable per logistische Regression gerechnet.

- „Erwerbsstatus nach einem Jahr“; hier ließen sich zunächst nur verschiedene Kategorien des Outcomes, nämlich „erwerbstätig“, „erwerbslos" und „berentet“, darstellen. Um den Einfluss auf diese Variable deutlich zu machen, wurde die Outcomevariable Erwerbsstatus dichotomisiert, und zwar unter dem Aspekt „keine Arbeit“ vs. „erwerbstätig“. Sie wurde auch hier als Dummy-Variable kodiert, „Erwerbstätigkeit“ mit "1“ und „NichtErwerbstätigkeit“ mit "0“. „Berentung“ wurde mit „0“, nicht erwerbstätig kodiert. Der Einfluss auf diese Outcomevariable wurde wieder per logistische Regression gerechnet.

Es ist noch zu bemerken, dass es immer wieder zu kleineren Stichproben gekommen ist, da sowohl Zugangs- bzw. Prädiktorvariablen sowie auch Outcomevariablen teilweise dem Statistik-Erfassungsprogramm „BADO“ entnommen wurden. Dieses war nicht immer vollständig ausgefüllt. Es wurde versucht, bei den für die Arbeit wesentlich interessierenden Variablen die Lücken anhand der Informationen aus den schriftlichen Entlassungsberichten zu schließen, was nicht immer befriedigend gelang; dann wurde der fehlende Wert hingenommen. Zudem basieren die Daten der Outcomevariablen „Abstinenz nach einem Jahr" und "Erwerbsstatus nach einem Jahr" auf der Katamneseerhebung; hier sind die fehlenden Daten außerdem dem Rücklauf zuzuschreiben.

In der Tabelle 2 werden die Outcomevariablen detailliert beschrieben. Als reguläre Entlassform (siehe Outcomevariable a) gelten die „regulär“ Entlassenen ( $N=304)$ sowie die „vorzeitig mit ärztlichem Einverständnis“ Entlassenen $(N=60)$. Alle anderen Entlassformen ( $\mathrm{N}=58$ ) werden als „irregulär entlassen“ gewertet. Ohne Angaben waren $(\mathrm{N}=12)$ Patienten. Beim "Therapieergebnis“ (Outcomevariable b) gelten nach Therapeuteneinschätzung $(\mathrm{N}=37)$ als erfolgreich behandelt, 
( $N=164)$ als gebessert, $(N=73)$ als unverändert und $(N=18)$ als während der Therapie verschlechtert. Hier liegt eine unvollständige Datenlage vor. Bei $(\mathrm{N}=142)$ Patienten wurde in der Basisdokumentation kein Therapieergebnis dokumentiert. Das Leistungsbild für die "letzte berufliche Tätigkeit“ > $6 \mathrm{~h}(\mathrm{~N}=286)$ sowie für den "allgemeinen Arbeitsmarkt" $>6 \mathrm{~h} \quad(\mathrm{~N}=338)$ korrelieren augenscheinlich hoch miteinander, etwas mehr $(N=52)$ sind aber für den allgemeinen Arbeitsmarkt vollschichtig leistungsfähig. In der Praxis ist für diese 52 Patienten anzunehmen, dass sie für eine berufliche Rehabilitation in Frage kommen. Wie schon dargestellt, wird der Effekt des Zugangs und der zugangsrelevanten Merkmale nur für das Leistungsbild für den allgemeinen Arbeitsmarkt (Outcomevariable c) gerechnet.

Die Abstinenzrate (Outcomevariable d) ist nach den Standards DGSS 3 und DGSS 4 (Deutschen Gesellschaft für Suchtforschung und Suchttherapie 2001) dargestellt. DGSS 3 bedeutet, dass nur die Antworter der katamnestischen Befragung in die Analyse mit eingehen, und zwar über alle Patienten, die ein Jahr zuvor entlassen wurden. In der Regel ist davon auszugehen, dass dies eine Überschätzung der tatsächlichen Abstinenzrate darstellt, da bei den Nichtantwortern vermutlich ein höherer Anteil an Rückfälligen vorliegt. Deshalb wird auch der DGSS 4 herangezogen, bei dem eben alle Nichtantworter auf die katamnestische Befragung als rückfällig gelten. Auch hier gehen alle im Jahr zuvor entlassenen Patienten in die Analyse mit ein, unabhängig von der Entlassform. Der Vollständigkeit halber sei noch hinzugefügt, dass der Standard DGSS 1 dem DGSS 3 (nur die Antworter werden einbezogen), der Standard DGSS 2 dem DGSS 4 (alle in einem Jahrgang entlassenen Patienten werden einbezogen) entspricht, mit dem Unterschied, dass bei DGSS 1 und 2 nur die regulär aus der Therapie entlassenen in die Analyse eingehen.

Bei der Überprüfung der spezifischen Hypothesen wird allerdings nur die Abstinenzrate nach DGSS 4 herangezogen, da diese den ungünstigsten Verlauf darstellt und nur bei dieser Auswertungsmethode alle Patienten berücksichtigt werden.

Der Rücklauf der katamnestischen Befragung stellt mit 233 Antwortern, bei 434 angeschriebenen ehemaligen Patienten (53,69\%), ein befriedigendes Ergebnis dar. 
Tabelle 2: Beschreibung der Outcomevariablen der Untersuchung auf Prädiktoren für den Behandlungserfolg von Alkoholabhängigen des Jahrgangs 2004 des Diakoniekrankenhauses Harz GmbH. Angaben der Häufigkeit in Absolutzahlen (N) und Prozent (\%)

\section{Legende:}

- Grau hinterlegt ist die Tabellenüberschrift

DGSS = Deutschen Gesellschaft für Suchtforschung und Suchttherapie

Die Daten zur Abstinenzrate nach DGSS 3 sowie zur Erwerbstätigkeit nach einem Jahr mit Darstellung der Berentungsrate wurden der Vollständigkeit halber hinzugefügt; sie dienen aber nicht als Outcomevariablen

\begin{tabular}{|c|c|c|c|}
\hline Outcomevariablen & & $\mathrm{N}$ & $\%$ \\
\hline \multirow{3}{*}{$\begin{array}{l}\text { Entlassform } \\
\text { [Outcomevariable a] }\end{array}$} & Regulär & 364 & 83,8 \\
\hline & Irregulär & 58 & 13,4 \\
\hline & Ohne Angaben & 12 & 2,8 \\
\hline \multirow{5}{*}{$\begin{array}{l}\text { Therapieergebnis } \\
\text { [Outcomevariable b] }\end{array}$} & Erfolgreich & 37 & 8,5 \\
\hline & Gebessert & 164 & 37,8 \\
\hline & Unverändert & 73 & 16,8 \\
\hline & Verschlechtert & 18 & 4,1 \\
\hline & Ohne Angaben & 142 & 32,7 \\
\hline \multirow{4}{*}{$\begin{array}{l}\text { Leistungsbild } \\
\text { für die letzte berufliche } \\
\text { Tätigkeit } \\
\text { [vergleichende Darstellung zur } \\
\text { Outcomevariable c] }\end{array}$} & $>6$ Stunden & 286 & 65,9 \\
\hline & $3-6$ Stunden & 19 & 4,4 \\
\hline & $<3$ Stunden & 102 & 23,5 \\
\hline & Ohne Angaben & 27 & 6,2 \\
\hline \multirow{4}{*}{$\begin{array}{l}\text { Leistungsbild } \\
\text { für den allgemeinen } \\
\text { Arbeitsmarkt } \\
\text { [Outcomevariable c] }\end{array}$} & $>6$ Stunden & 338 & 77,9 \\
\hline & $3-6$ Stunden & 22 & 5,1 \\
\hline & $<3$ Stunden & 46 & 10,6 \\
\hline & Ohne Angaben & 28 & 6,5 \\
\hline \multirow{4}{*}{$\begin{array}{l}\text { Abstinenzrate (DGSS 3) } \\
\text { - nur die Antworter werden } \\
\text { gerechnet - }\end{array}$} & Abstinent & 163 & 70,0 \\
\hline & Abstinent nach Rückfall & 10 & 4,3 \\
\hline & rückfällig & 55 & 23,6 \\
\hline & Keine Angaben & 5 & 2,1 \\
\hline \multirow{4}{*}{$\begin{array}{l}\text { Abstinenzrate (DGSS 4) } \\
\text { - Nicht-Antworter werden als } \\
\text { rückfällig gerechnet } \\
\text { [Outcomevariable d] }\end{array}$} & Abstinent & 163 & 37,6 \\
\hline & Abstinent nach Rückfall & 10 & 2,3 \\
\hline & rückfällig & 256 & 59,0 \\
\hline & Keine Angaben & 5 & 1,2 \\
\hline \multirow{3}{*}{$\begin{array}{l}\text { Erwerbstätigkeit nach einem } \\
\text { Jahr } \\
\text { Arbeit ja/nein } \\
\text { [Outcomevariable e] }\end{array}$} & Erwerbstätig & 83 & 19,1 \\
\hline & Nicht erwerbstätig & 118 & 27,2 \\
\hline & Ohne Angaben & 233 & 53,7 \\
\hline \multirow{4}{*}{$\begin{array}{l}\text { Erwerbstätigkeit nach einem } \\
\text { Jahr } \\
\text { Berentung gesondert dargestellt }\end{array}$} & Erwerbstätig & 83 & 19,1 \\
\hline & Erwerbslos & 75 & 17,3 \\
\hline & Berentet & 46 & 10,6 \\
\hline & Ohne Angaben & 233 & 53,7 \\
\hline
\end{tabular}


Die „Erwerbstätigkeit nach einem Jahr" wird in Tabelle 2 zum Einen nach Erwerbstätigkeit „ja/nein“ (Outcomevariable e) dargestellt, zum Anderen, der Übersichtlichkeit halber, nach der Erwerbssituation insgesamt. Hier können selbstverständlich nur die Katamneseantworter in die statistische Analyse eingehen. 


\section{Ergebnisse}

\subsection{Darstellung der Ergebnisse}

Die folgende Tabelle 3 stellt alle relevanten Ergebnisse in komprimierter Form dar. In den einzelnen Spalten finden sich die Ergebnisse unterteilt nach den spezifischen Hypothesen a bis e.

Tabelle 3: Gesamtübersicht über die Regressionsanalysen der Hypothesen 1 und 2 bezogen auf die Outcomevariablen a bis e in der Untersuchung auf Prädiktoren für den Behandlungserfolg von Alkoholabhängigen des Jahrgangs 2004 des Diakoniekrankenhauses Harz GmbH

Legende:

- $\quad$ "Hyp" = Hypothese

- $\quad$ "log“ = logistische Regression; „lin“ = lineare Regression

- „Anteil „1““ gibt an, wie oft und mit welchem prozentualen Anteil die dichotome Outcomevariable den Wert "1" angenommen hat (bei der linearen Regression werden Mittelwert (MW) und Standardabweichung (SD) angegeben)

${ }, \mathrm{R}^{2 \text { ، }}=$ Anteil der aufgeklärten Varianz durch die im Modell zusammengefassten unabhängigen Variablen

Als Dummy-Variablen gingen in die Berechnung ein: „Auflage“ („Auflage“ erhielt den Wert "1“), „Geschlecht" („weiblich“ = „1“), „Wiederaufnahme“ (,Ja“ = „1“), ,Zuvor suchtbezogene Hilfe (erhalten)“ („Ja“ = „1“)

In Dummy-Variablen "umgewandelte kategoriale Variablen sind: „Motivationslage“ (Referenzkategorie (=RK) „Eigenmotiviert"), „Dauer_Arbeitslosigkeit“ (RK „Arbeitslosigkeit < 1 Monat"), „Erwerbssituation_Anfang“ (RK „erwerbstätig“), „Schulabschluss“ (RK „Abitur/(Fach)Hochschulreife“), „Kostenträger“ (RK „BfA = Bundesversicherungsanstalt für Angestellte“), „Familienstand“ (RK „verheiratet, zusammenlebend“)

„c.l.-Skala" = Skala zur Erfassung cerebraler Insuffizienzen

„PSSI“ = Persönlichkeits-Stil-und-Störungs-Inventar

"LVA" = Landesversicherungsanstalt, der frühere Name der regionalen Rentenversicherungen

Die Darstellung in den Spalten erfolgt in Form des Signifikanzniveaus gemäß üblicher Konnotation: ${ }^{*}=p<0,05 ;{ }^{* *}=p<0,01 ;{ }^{* * *}=p<0,001$

$(+)$ und (-) geben die Richtung der Effekte wieder

\begin{tabular}{|c|c|c|c|c|c|}
\hline & $\begin{array}{l}\text { Hyp } 1 \text { a+ } 2 \text { a } \\
\text { Entlassform }\end{array}$ & $\begin{array}{c}\text { Hyp } 1 \text { b + } 2 \text { b } \\
\text { Therapie- } \\
\text { ergebnis }\end{array}$ & $\begin{array}{l}\text { Hyp } 1 \text { c + } 2 \text { c } \\
\text { Leistungsbild } \\
\text { allgemein }\end{array}$ & Hyp $1 d+2 d$ & Hyp 1 e +2 e \\
\hline N (Anzahl der Fälle) & 268 & 230 & 259 & 265 & 127 \\
\hline Methode & $\log$ & $\operatorname{lin}$ & lin & $\log$ & $\log$ \\
\hline Anteil "1" bzw. MW / SD & $239(89,2 \%)$ & $2,27 / 0,75$ & $5,27 / 0,64$ & $106(40 \%)$ & $55(43,3 \%)$ \\
\hline $\begin{array}{l}\mathrm{R}^{2} \\
\text { richtige Klassifikation durch }\end{array}$ & 0,534 & 0,311 & 0,317 & 0,239 & 0,817 \\
\hline logistische Regression & $94,40 \%$ & --- & --- & $71,70 \%$ & $96,10 \%$ \\
\hline Auflage = "Auflage" & 0,142 & 0,609 & 0,908 & 0,204 & 0,954 \\
\hline Motivationslage & $0,000^{* * *}$ & & & $0,02^{*}$ & 0,09 \\
\hline Ambivalenz-Motivation & $0,004^{* *}(-)$ & 0,421 & 0,225 & $0,005^{* *}(-)$ & $0,03^{*}(-)$ \\
\hline Fremd-Motivation & $0,000 * * *(-)$ & $0,014 *(+)$ & 0,437 & 0,72 & 0,922 \\
\hline Alter bei Aufnahme & $0,043^{*}(-)$ & 0,334 & 0,398 & 0,167 & 0,756 \\
\hline Wartezeit & 0,869 & 0,254 & 0,462 & 0,861 & $0,006^{*}(-)$ \\
\hline
\end{tabular}


Fortsetzung Tabelle 3: Gesamtübersicht über die Regressionsanalysen der Hypothesen 1 und 2 bezogen auf die Outcomevariablen a bis $e$ in der Untersuchung auf Prädiktoren für den Behandlungserfolg von Alkoholabhängigen des Jahrgangs 2004 des Diakoniekrankenhauses Harz GmbH

\begin{tabular}{|c|c|c|c|c|c|}
\hline Geschlecht = "weiblich" & 0,730 & $0,010^{*}(+)$ & 0,728 & 0,275 & 0,601 \\
\hline $\begin{array}{l}\text { Wortschatz-Intelligenz- } \\
\text { Quotient }\end{array}$ & 0,706 & 0,320 & 0,630 & 0,889 & $0,014^{*}(+)$ \\
\hline Verbalgedächtnis & 0,139 & 0,422 & $0,035^{*}(-)$ & 0,642 & $0,008^{* *}(+$ \\
\hline cl-Skala & 0,649 & 0,276 & 0,607 & 0,818 & 0,092 \\
\hline PSSI (Selbstunsicherheit) & 0,667 & 0,362 & 0,444 & 0,544 & 0,279 \\
\hline PSSI (spontan-borderline) & 0,409 & 0,195 & 0,909 & 0,33 & 0,279 \\
\hline Abhängigkeitsdauer & 0,341 & $0,013^{*}(+)$ & 0,630 & 0,532 & $0,009^{* *}(-)$ \\
\hline $\begin{array}{l}\text { Dauer_Arbeitslosigkeit } \\
\text { Dauer_Arbeitslosigkeit: } \\
1 \text { bis } 12 \text { Monate } \\
\text { Dauer_Arbeitslosigkeit: } \\
>12 \text { Monate }\end{array}$ & $\begin{array}{l}0,029^{*} \\
0,025^{*}(-) \\
0,008^{* *}(-)\end{array}$ & $\begin{array}{l}0,872 \\
0,688\end{array}$ & $\begin{array}{l}0,654 \\
0,384\end{array}$ & $\begin{array}{l}0,861 \\
0,705\end{array}$ & $\begin{array}{l}0,008^{* *} \\
0,002^{* *}(-) \\
0,002^{* *}(-)\end{array}$ \\
\hline Erwerbssituation_Anfang & $0,014^{*}$ & & & 0,814 & 0,224 \\
\hline Erwerbssituation: erwerbslos & $0,002^{* *}(+)$ & 0,726 & 0,079 & 0,466 & $0,036^{*}(+)$ \\
\hline Erwerbssituation: berentet & 1,000 & 0,198 & $0,000^{* * *}(+)$ & 0,649 & 1 \\
\hline Erwerbssituation: Sonstiges & $0,009^{* *}(+)$ & 0,516 & $0,050 *(+)$ & 0,888 & 0,989 \\
\hline Schulabschluss & 0,339 & & & 0,776 & 0,404 \\
\hline $\begin{array}{l}\text { Schule: keine Angaben } \\
\text { Schule: ohne }\end{array}$ & 1,000 & & & 1 & 1 \\
\hline & 1,000 & 0,823 & 0,413 & 0,208 & 1 \\
\hline & 1,000 & 0,079 & 0,374 & 0,856 & $0,028^{*}(+)$ \\
\hline & 0,173 & 0,222 & $0,013^{*}(+)$ & 0,495 & 0,814 \\
\hline $\begin{array}{l}\text { Polytechnische Oberschule } \\
\text { Schule: Anderer }\end{array}$ & 0,110 & 0,437 & 0,141 & 0,795 & 0,458 \\
\hline Schulabschluss & $0,009^{* *}(-)$ & & & 0,794 & 1 \\
\hline Kostenträger & 0,987 & & & 0,843 & 0,163 \\
\hline Kostenträger: Andere & 0,890 & 0,525 & 0,175 & 0,496 & 0,356 \\
\hline $\begin{array}{l}\text { Kostenträger: Andere LVAen } \\
\text { Kostenträger: }\end{array}$ & 1,000 & 0,267 & 0,882 & 0,828 & 0,766 \\
\hline $\begin{array}{l}\text { Krankenkassen } \\
\text { Kostenträger: }\end{array}$ & 1,000 & 0,994 & 0,184 & 0,659 & 1 \\
\hline $\begin{array}{l}\text { Bahn/Knappschaft/See } \\
\text { Kostenträger: LVA Sachsen- }\end{array}$ & 0,808 & 0,971 & 0,827 & 0,203 & 0,118 \\
\hline $\begin{array}{l}\text { Anhalt } \\
\text { Kostenträger: LVA }\end{array}$ & 0,985 & 0,795 & 0,272 & 0,355 & 0,997 \\
\hline Thüringen & 0,427 & 0,525 & 0,960 & 0,43 & $0,004^{* *}(+$ \\
\hline Familienstand & 0,094 & & & 0,249 & 0,079 \\
\hline $\begin{array}{l}\text { Familienstand: verheiratet - } \\
\text { getrennt lebend }\end{array}$ & 0,233 & 0,805 & 0,173 & 0,216 & 0,124 \\
\hline Familienstand: ledig & $0,009^{* *}(-)$ & 0,082 & 0,082 & 0,965 & $0,028^{*}(+)$ \\
\hline Familienstand: geschieden & $0,038^{*}(-)$ & 0,422 & 0,592 & 0,156 & $0,004^{* *}(+$ \\
\hline Familienstand: verwitwet & 1,000 & & 0,295 & 0,286 & 1 \\
\hline
\end{tabular}


Fortsetzung Tabelle 3: Gesamtübersicht über die Regressionsanalysen der Hypothesen 1 und 2 bezogen auf die Outcomevariablen a bis $\mathrm{e}$ in der Untersuchung auf Prädiktoren für den Behandlungserfolg von Alkoholabhängigen des Jahrgangs 2004 des Diakoniekrankenhauses Harz GmbH

\begin{tabular}{|c|c|c|c|c|c|}
\hline Wiederaufnahme = "Ja" & 0,606 & 0,827 & 0,860 & 0,057 & 0,83 \\
\hline Zuvor suchtbez. Hilfe = "Ja" & 0,055 & 0,994 & 0,116 & 0,138 & 0,49 \\
\hline $\begin{array}{l}\begin{array}{l}\text { Körperbezogene } \\
\text { Komorbidität }\end{array} \\
\end{array}$ & 0,467 & 0,837 & 0,087 & 0,287 & 0,334 \\
\hline Psychische Erkrankungen & 0,461 & 0,485 & 0,173 & 0,291 & $0,049^{*}(+)$ \\
\hline $\begin{array}{l}\text { Alkoholbezogene } \\
\text { Erkrankungen }\end{array}$ & $0,039^{*}(+)$ & 0,785 & 0,060 & 0,358 & 0,583 \\
\hline $\begin{array}{l}\text { Zweite Suchtdiagnose (ohne } \\
\text { Tabakabhängigkeit) }\end{array}$ & 0,671 & 0,085 & $0,050 *(+)$ & 0,176 & 0,505 \\
\hline Tabakabhängigkeit & 0,677 & 0,914 & 0,755 & 0,632 & 0,056 \\
\hline Persönlichkeitsstörung & 0,918 & 0,601 & 0,932 & 0,458 & $0,022^{*}(+)$ \\
\hline Intelligenzminderung & 1,000 & 0,609 & 0,137 & 0,535 & 1 \\
\hline Konstante & $0,010^{*}$ & 0,067 & $0,000^{\star \star \star}$ & 0,656 & $0,026^{*}$ \\
\hline
\end{tabular}

\section{Zugang und Entlassform:}

\section{Ergebnisse zu den spezifischen Hypothesen 1 a und 2 a}

Bezüglich der Analyse hinsichtlich des Einflusses von Zugangswegen und Zugangsvariablen auf die Entlassform wurden 268 Patienten und damit 61,8\% aller Fälle einbezogen. Von diesen 268 Patienten wurden 239 regulär entlassen, 29 irregulär. Die restlichen 166 Patienten konnten bei der Analyse nicht berücksichtigt werden, da bei innen in irgendeinem Punkt des Datensatzes unvollständige Informationen vorlagen.

Es sei noch einmal an die Kodierung der abhängigen Variablen erinnert, irregulär Entlassene wurden mit „0“ kodiert, regulär Entlassene mit „1“.

Mit Nagelkerkes R-Quadrat von 0,534 zeigt sich hier ein hoher Wert für die Effektstärke des Modells, da die zusammengefassten Variablen immerhin 53,4\% der gemeinsamen Varianz aufklären. Daraus lässt sich auch ableiten, dass die Vorhersagekraft der zusammengefassten unabhängigen Variablen insgesamt auf die Entlassform relativ hoch ist. Dies bestätigt sich auch in der nachfolgenden Klassifizierungsgüte, die im Folgenden noch einmal erläutert sei: Der Anteil der 
regulär entlassenen Patienten in der Untersuchung entspricht 239 von 268 Patienten und damit 89,2\%. Bei zufälliger Zuordnung wären dann 80,7\% richtig zugeordnet. Durch die im Modell zusammengefassten Variablen konnten aber von 268 Fällen 253 richtig klassifiziert werden, d. h. 94,4\% der Patienten wurden durch die untersuchten Zugangsvariablen der richtigen Entlassform zugeordnet. Mit dieser Trefferquote ist von einer hohen Klassifikationsgüte auszugehen.

Die Ergebnisse lassen sich wie folgt zusammenfassen: Es zeigt sich ein signifikanter Einfluss der folgenden Variablen auf die Entlassform:

- „Ambivalenzmotivation“

- „Fremdmotivation“

- „Alter bei Aufnahme“

- „Dauer_Al: 1 bis 12 Monate“

- „Dauer_Al: > 12 Monate“

- „Anderer Schulabschluss“

- „Familienstand: ledig“

- „Familienstand: geschieden“

- „Erwerbssituation_Anfang“

- „Erwerbssituation erwerbslos“

- „Erwerbssituation sonstige“

- „Alkoholfolgediagnose“

Innerhalb der Motivationslage, d. h. bezogen auf die Referenzkategorie Eigenmotivation, findet sich für die ambivalent Motivierten eine verminderte Wahrscheinlichkeit für eine reguläre Entlassung. Dies gilt ebenso für die Fremdmotivierten.

Hinsichtlich des Alters bei Aufnahme ergibt sich eine verminderte Wahrscheinlichkeit für die reguläre Entlassung, je älter die Patienten sind.

Sowohl die Kurzzeit- als auch die Langzeit-Arbeitslosen weisen eine niedrigere Quote für eine reguläre Entlassung auf, als die Erwerbstätigen. 
Innerhalb der Kategorie Schulbildung findet sich für ",anderer Schulabschluss“ eine verminderte Wahrscheinlichkeit für reguläre Entlassung, allerdings ohne signifikanten Einfluss der Hauptkategorie „Schulabschluss“.

Die Variablen „Familienstand: ledig“ und „Familienstand: geschieden“ weisen eine niedrigere Quote für eine reguläre Entlassung auf.

Bezüglich der „Erwerbssituation_Anfang“ ist die Situation der Erwerbslosen, der Berenteten und der sonstigen Erwerbssituationen nicht klar zu deuten, ein Zusammenhang zur Entlassform besteht aber (siehe oben).

Das Merkmal „Alkoholfolgeerkrankung“ weist eine höhere Quote für eine reguläre Entlassung auf.

\section{Zugang und Therapieergebnis:}

\section{Ergebnisse zu den spezifischen Hypothesen $\mathbf{1}$ b und $\mathbf{2}$ b}

Die abhängige Variable des vom Therapeuten eingeschätzten Therapieergebnisses von „verschlechtert“ ( $=4)$ bis "erfolgreich“ ( $(=1)$ ist metrischer Natur. Aus diesem Grund wird hier die multiple lineare Regressionsanalyse durchgeführt. Es gingen 230 Fälle in die Analyse ein.

In der Modellzusammenfassung zeigt sich mit einem Wert für R-Quadrat von 0,311, dass etwa $31 \%$ des Therapieergebnisses durch die Zusammenfassung der unabhängigen Variablen bestimmt werden. Der Einfluss der untersuchten Variablen auf das Therapieergebnis ist hochsignifikant $(p<0.001, F=2,204)$ [siehe Tabelle $10 \mathrm{im}$ Anhang 5].

Es zeigt sich ein signifikanter Einfluss der folgenden Variablen auf die vom Therapeuten gegebene Einschätzung hinsichtlich des Therapieergebnisses:

- „Fremdmotivation“ (auch hier wieder im Vergleich zur Referenzkategorie „Eigenmotivation")

- "Geschlecht = weiblich“ (Frauen im Vergleich zu Männern)

- „Abhängigkeitsdauer“ (je länger die Abhängigkeit besteht) 
Die drei hier genannten Variablen sind die einzigen, die signifikante Zusammenhänge zu der Outcomevariablen "Therapieergebnis“ aufweisen. Hierbei wirken sie sich alle negativ aus.

\section{Zugang und Leistungsbild:}

\section{Ergebnisse zu den spezifischen Hypothesen $1 \mathrm{c}$ und $2 \mathrm{c}$}

Auch hier waren die abhängigen Variablen metrischer Natur, deshalb nachfolgend die Berechnungen zur multiplen linearen Regression.

Hinsichtlich des Merkmals „Leistungsbild für den allgemeinen Arbeitsmarkt“ gingen $(\mathrm{N}=259)$ in die Analyse ein.

Die durch die unabhängigen Variablen aufgeklärte Varianz von $31,7 \%$ ist mäßiggradig hoch einzustufen, dennoch ergibt sich ein hochsignifikanter Zusammenhang zwischen Zugangsvariablen und dem Leistungsbild für den allgemeinen Arbeitsmarkt ( $p<0.001, F=2,535)$ [siehe Tabelle 013 im Anhang 5].

Es zeigt sich ein signifikanter Einfluss der folgenden Variablen auf das "Leistungsbild für den allgemeinen Arbeitsmarkt":

- Erwerbssituation berentet (im Verhältnis zu „erwerbstätig“, ebenso:)

- Erwerbssituation sonstige

- Hauptschulabschluss (im Verhältnis zu (Fach-)Hochschulreife)

- zweite Suchtdiagnose

- Verbalgedächtnis (zur Gesamtheit aller unhabhängigen Variablen)

Die ersten vier beschriebenen Variablen wirken sich negativ auf das „Leistungsbild für den allgemeinen Arbeitsmarkt" aus.

Das Vorzeichen des Effekts beim Merkmal „Verbalgedächtnis“ ist negativ, d. h. ein gutes Verbalgedächtnis wirkt sich positiv auf die abhängige Variable aus.

\section{Zugang und Abstinenzrate:}

\section{Ergebnisse zu den spezifischen Hypothesen $1 \mathrm{~d}$ und $2 \mathrm{~d}$ :}

Bezüglich der Analyse hinsichtlich des Zusammenhangs zwischen Zugangswegen und Zugangsvariablen auf die „Abstinenz nach einem Jahr“ wurden 265 Patienten 
und damit 61,1 \% aller Fälle mit einbezogen. 106 der 265 Fälle waren nach DGSS 4 (alle Nichtantworter gelten als rückfällig) abstinent. Dies entspricht $40 \%$ stabil Abstinenten.

In der Modellzusammenfassung ist hier Nagelkerkes R-Quadrat immerhin noch 0,239. D. h., dass $23,9 \%$ gemeinsamer Varianz durch das Modell aufgeklärt werden. Die Vorhersagekraft der im Modell zusammengefassten Variablen ist aber nach einem Jahr nicht so groß, wie beispielsweise auf die Entlassform.

Die Klassifizierungsgüte ist hier hoch: Durch die im Modell zusammengefassten Variablen wurden $71,7 \%$ der Patienten durch die untersuchten Zugangsvariablen dem Merkmal „Abstinenz nach einem Jahr“ richtig zugeordnet.

Es zeigt sich ein signifikanter Einfluss der folgenden Variable auf die „Abstinenz nach einem Jahr":

- „Ambivalenzmotivation“

Die „Ambivalenzmotivation“ wirkt sich im Vergleich zur Eigenmotivation negativ auf die Abstinenz aus.

Die Fremdmotivation im Vergleich zur Eigenmotivation zeigte dagegen keinen signifikanten Effekt auf die Abstinenzrate.

\section{Zugang und Erwerbssituation nach einem Jahr:}

\section{Ergebnisse zu den spezifischen Hypothesen 1 e und 2 e:}

Bezüglich der „Erwerbssituation nach einem Jahr“ wurde der Einfluss der unabhängigen Variablen daraufhin überprüft, ob jemand nach einem Jahr erwerbstätig ist.

Bezüglich der Analyse hinsichtlich des Einflusses von Zugangswegen und Zugangsvariablen auf das Merkmal „Arbeit nach einem Jahr" flossen lediglich ( $N=$ 127) Fälle ein. Dies ist auf den Fragebogen-Rücklauf und auf lückenhafte Dokumentation zurückzuführen. 
In der Modellzusammenfassung ist hier Nagelkerkes R-Quadrat von 0,817 hoch. $81,7 \%$ gemeinsame Varianz werden durch die zusammengefassten Variablen aufgeklärt.

Entsprechend ist auch die Klassifizierungsgüte hoch: Durch die im Modell zusammengefassten Variablen wurden $96,1 \%$ der Patienten durch die untersuchten Zugangsvariablen dem Merkmal „Arbeit nach einem Jahr“ richtig zugeordnet.

Es zeigt sich ein signifikanter Einfluss der folgenden Variablen auf das Merkmal „Arbeit nach einem Jahr"

- „Ambivalenzmotivation“

- „Wartezeit“

- „Abhängigkeitsdauer“

- „Kurzzeitarbeitslosigkeit“

- „Langzeitarbeitslosigkeit“

- „Wortschatz-IQ“

- „Verbalgedächtnis“

- „Erwerbssituation erwerbslos“

- "Sonderschulabschluss“

- „Kostenträger LVA Thüringen“

- „Familienstand ledig“

- „Familienstand geschieden“

- „Komorbidität „psychische Erkrankungen“

- „Komorbidität „Persönlichkeitsstörungen“

Die Variable „Wartezeit": wirkt sich negativ auf das Merkmal „Erwerbssituation ein Jahr nach Entlassung" aus.

Die „Abhängigkeitsdauer“ wirkt sich negativ aus.

Die Kategorie „Dauer der Arbeitslosigkeit“ vor der Therapie zeigte signifikante Zusammenhänge. Sowohl vorherige „Kurzzeit-Arbeitslosigkeit“ als auch „Langzeit- 
Arbeitslosigkeit“ wirkten sich negativ auf die "Erwerbssituation nach einem Jahr“ aus.

Eine hohe „verbale Intelligenz“ (Abschätzung des „Wortschatz-IQ“) wirkt sich positiv aus.

Eine „gute Merkfähigkeit“ wirkt sich positiv aus

Die Merkmale „ledig“ und "geschieden“ wirken sich hinsichtlich der Outcomevariablen „Erwerbssituation nach einem Jahr“ positiv aus.

Des Weiteren zeigten sich für einzelne Variablen signifikante Effekte, ohne dass sich diese Effekte in der Gesamtvariable erkennen ließen:

„Erwerbslosigkeit“ im Vergleich zur „Erwerbstätigkeit“

„Sonderschulabschluss“ im Vergleich zur „Fachhochschulreife“

der Kostenträger „LVA Thüringen“ im Vergleich zur „BfA“.

Alle letztgenannten Effekte gingen in Richtung zu mehr „Erwerbstätigkeit nach einem Jahr", d. h. sie wirkten sich positiv aus.

Die Variablen „Komorbidität psychische Erkrankungen“ und „Komorbidität Persönlichkeitsstörungen" wirken sich beide positiv aus.

\section{Vergleich der Ergebnisse mit den RMK-Forschungsergebnissen}

Beim Vergleich der hiesigen Ergebnisse mit den in der RMK-Forschung definierten sechs Subdimensionen:

- „substanzenbezogene Beeinträchtigung“

- "psychische Symptomatik“

- „persönliche Ressourcen“

- „arbeitsbezogenes Erleben und Verhalten“

- „Alter und Arbeitslosigkeit“

- „soziale Unterstützung“ 
zeigt sich, dass sich die signifikanten Zusammenhänge, die in der vorliegenden Arbeit beschrieben werden, nur sehr begrenzt in den Ergebnissen der RMKForschung widerspiegeln.

Lediglich die sozialmedizinischen Elemente werden in den Subdimensionen „Erwerbsproblematik“ und „arbeitsbezogenes Erleben und Verhalten“ spezifisch aufgegriffen.

Einige Parameter aus dem Feld der hier relevanten kognitiven Leistungstestung dürften sich in den Subdimensionen „psychische Symptomatik“ und „persönliche Ressourcen“ wiederfinden.

\subsection{Ergebniszusammenfassung}

Betrifft Ergebnisse zur spezifischen Hypothese 1:

Die Motivationslage spielt in allen untersuchten Bereichen eine signifikante Rolle. Lediglich das „Leistungsbild allgemeiner Arbeitsmarkt“, welches nach ICF vom Arzt getätigte gutachterliche Beschreibung ist, ist von der Motivationsfrage losgelöst.

Betrifft Ergebnisse zur spezifischen Hypothese 2:

Das „Alter bei Aufnahme“ weist Zusammenhänge zur „Entlassform“ auf.

Die „Wartezeit“ auf einen Therapieplatz weist Zusammenhänge zur „Erwerbssituation ein Jahr nach Entlassung“ auf.

Das „Geschlecht“ weist Zusammenhänge zum „Therapieergebnis“ auf.

Der „Wortschatz IQ“ weist Zusammenhänge zur „Erwerbssituation ein Jahr nach Entlassung" auf.

Das „Verbalgedächtnis“ weist Zusammenhänge zum „Leistungsbild für den allgemeinen Arbeitsmarkt“ und zur „Erwerbssituation ein Jahr nach Entlassung“ auf.

Die „Abhängigkeitsdauer“ vor Therapie weist Zusammenhänge zum „Therapieergebnis“ und zur „Erwerbssituation ein Jahr nach Entlassung“ auf.

Die „Dauer der Arbeitslosigkeit“ vor Therapie weist Zusammenhänge zur „Entlassform“ und zur „Erwerbssituation ein Jahr nach Entlassung“ auf.

Die „Erwerbssituation“ vor Therapie weist Zusammenhänge zur „Entlassform“ und zum „Leistungsbild für den allgemeinen Arbeitsmarkt“ auf. 
Der „Schulabschluss“ weist Zusammenhänge zum „Leistungsbild für den allgemeinen Arbeitsmarkt" und zur "Erwerbssituation ein Jahr nach Entlassung“ auf.

Der „Kostenträger LVA Thüringen“ weist Zusammenhänge zur „Erwerbssituation ein Jahr nach Entlassung" auf.

Der „Familienstand“ weist Zusammenhänge zur „Entlassform“ und zur „Erwerbssituation ein Jahr nach Entlassung“ auf.

„Psychische Diagnose“ und „Persönlichkeitsdiagnose“ weisen Zusammenhänge zur „Erwerbssituation ein Jahr nach Entlassung“ auf.

Die „Alkoholfolgediagnose“ weist Zusammenhänge zur „Entlassform“ auf.

Die „zweite Suchtdiagnose“ weist Zusammenhänge zum „Leistungsbild für den allgemeinen Arbeitsmarkt" auf.

Es gilt demzufolge:

Einzelne Zugangsvariablen weisen Zusammenhänge $z u$ den untersuchten Outcomevariablen auf und können als Prädiktoren für das Outcome gelten.

Betrifft Haupthypothese 3:

Es finden sich einige Überschneidungbereiche zwischen den Ergebnissen der vorliegenden Arbeit und den definierten Subdimensionen der RMK-Forschung. Die wesentlichen Ergebnisse beider Betrachtungen sind aber weder identisch noch in hohem Maße deckungsgleich. 


\section{Diskussion}

In der vorliegenden Arbeit wurden mehrere Fragestellungen (siehe Punkt 1.1) beleuchtet. Sie sollen hier noch einmal inhaltlich zusammengefasst formuliert und betrachtet werden:

1) Welche Bedeutung hat die Motivation eines Patienten zu Beginn der Alkohollangzeittherapie mit dem Therapieergebnis? Das Verhältnis von eigenmotivierten Patienten $\mathrm{zu}$ ambivalent motivierten Patienten und fremdmotivierten Patienten sollte beleuchtet werden.

2) Spielt die Zugangsform zur Rehabilitationseinrichtung bei der Behandlung von Alkoholabhängigen eine Rolle hinsichtlich des Behandlungsergebnisses?

3) Können aus den in Punkt 2.4.5 beschriebenen Zugangsvariablen Prädiktoren für das Therapieoutcome bei der Behandlung von Alkoholabhängigen abgeleitet werden? Inwieweit sind diese in den Fallgruppen der Rehabilitanden-Management-Kategorien abgebildet? Inwieweit fokussiert die aktuelle Rehabilitationsforschung problemadäquat?

\section{$\mathrm{zu} 1)$}

Aus den Untersuchungsergebnissen geht eindeutig hervor, dass der Motivation zu Beginn der Therapie die entscheidende Rolle für das therapeutische Outcome zukommt. Insbesondere das Phänomen der „ambivalenten Therapiemotivation“ taucht als problematischer Faktor auf. Es soll deshalb an dieser Stelle gezielt betrachtet werden (Grosse Holtforth und Michalak 2009):

Bei ambivalenter Therapiemotivation innerhalb eines therapeutischen Prozesses stellt sich dem Therapeuten regelmäßig die Frage: Kann oder will der Patient die notwendigen Schritte seiner gesundheitsfördernden Veränderung nicht unternehmen? Entweder muss im faktischen Bereich nachgelernt werden (der Patient kann nicht) oder im motivationalen Bereich (der Patient will nicht). Sehr häufig liegt keine stabile Krankheitseinsicht vor. 
Eine weitere Form der Ambivalenz findet sich bei Patienten mit offensichtlichem Leidensdruck, d. h. mit klarer Problemeinsicht, aber dennoch mit mangelnder Veränderungsbereitschaft. Bei Suchtpatienten beispielsweise findet sich diese Form sehr häufig. Dann werden häufig in der Therapie Fertigkeiten erarbeitet und erlernt, kommen aber im Alltag des Patienten nicht zur Anwendung.

Nach der Konflikttheorie finden sich konflikthafte Bereiche im Erleben einer Person, die ambivalentes Verhalten initieren. Diese intrapsychischen oder intrapersonellen Konflikte bedingen eine stabile Unentschiedenheit zwischen Verhaltensweisen, so genannten motivationalen Konflikten. Grosse Holtforth und Michalak übersetzen P. E. Meehls Definition der Ambivalenzen als „Existenz simultaner oder schnell austauschbarer positiver und negativer Gefühle gegenüber demselben Objekt oder gegenüber der selben Aktivität" (Grosse Holtforth und Michalak 2009, Seite 632).

In der Praxis finden sich Ambivalenzen vor allem bezüglich der Aufnahme einer Therapie, der nötigen Veränderung in einer Therapie oder der Durchführung von veränderungsorientierten Verhaltensweisen in einer Therapie.

Wenn ein Patient beispielsweise das Annehmen von Hilfe grundsätzlich als Schwäche oder Versagensleistung versteht, wird er bezüglich der Therapieaufnahme ambivalent empfinden. Auch wenn Symptomreduktion die Gefährdung einer Beziehung oder eines Systems bedeutet, findet sich ein Patient in der Ambivalenz. Bei Veränderungswunsch, aber ausgeprägter Angst vor dem Erlernen einer Veränderung (beispielsweise Exposition bei Angststörung), tritt Ambivalenz auf. Die hier beschriebenen Ambivalenzen sind therapiebezogen.

Im alltäglichen Bereich, beispielsweise in Partnerschaften, ergibt sich häufig ein Ambivalenzkonflikt zwischen Autonomiewunsch und Verlustangst. Ein solcher innerer Ambivalenzkonflikt kann rasch als Widerstand in der Therapie erlebt werden; vorrangiges Ziel wäre dann die Hilfe zur Entscheidungsfähigkeit. Hierbei muss dem Behandler klar sein, dass für den Betroffenen eine Entscheidung an sich angstmachend besetzt ist, da, unabhängig vom Ergebnis der Entscheidung, positive wie auch negative Konsequenzen zu tragen sind. 
In der Suchtentwicklung bzw. in der Entwicklung des Problembewusstseins von Suchtkranken kann man die Stufen des transtheoretischen Modells (Prochaska und DiClemente 2005) schlüssig nachvollziehen.

Die erste Phase der "Sorglosigkeit" wird durch mangelndes oder sehr geringes Problembewusstsein und resultierend fehlender Veränderungsabsicht gekennzeichnet. Die zweite Stufe des „zunehmenden Problembewusstseins“ zeigt sich durch Problemwahrnehmung, aber noch ohne beabsichtigte Verhaltensänderung. Auf der dritten Stufe der „Vorbereitung“ werden konkrete Absichten und Vorsätze der Verhaltensänderung gebildet. Diese werden in der vierten Stufe der „Handlung“ beginnend umgesetzt. Auf Stufe fünf, der „Aufrechterhaltung“, werden Erkenntnisse und bisher Umgesetztes stabilisiert. In der letzten Stufe werden die Veränderungen zum „Abschluss“ gebracht. Der vermutlich wesentlichste Entwicklungsbereich ist zwischen Bewusstwerdung und Vorbereitung zu sehen; in dieser Phase wird die Entscheidung getroffen, den Zustand der Ambivalenz zu verlassen.

Die Ambivalenz ist also unabhängig von ihrer Dauer quasi einer permanenten Entscheidungssituation ohne Ergebnis gleichzusetzen. Die Vermeidung der Verhaltensänderung, bei gleichzeitigem Erleiden der vorherrschenden Situation, ist trotz teilweiser subjektiv unerträglich empfundener innerer Spannung die Beste aller Optionen.

Die eigentliche Bearbeitung von Ambivalenzen ist ein therapeutisch schwieriger und teilweise langwieriger Prozess. Wenn es gelingen würde, in einem Assessment für Rehabilitationssteuerung und Fallgruppenzuordnung Ambivalenzen $z u$ erkennen und $z u$ benennen, d. h., wenn bei der Fallgruppendefinition das Ausmaß oder besser die Stabilität der Ambivalenz benannt werden könnte, wäre eines der massivsten Therapiehindernisse bei der Rehabilitation und der Rehabilitationssteuerung besser berücksichtigt. Inwieweit bereits im Vorfeld inhaltliche Ambivalenzen beschrieben werden können, d. h., Objekte, welche die Ambivalenzen bedingen, detektiert werden können, bleibt zu prüfen. Gegebenenfalls würde es eher der Praxis entsprechen, wenn die Kliniken, die sich auf die Bearbeitung von Ambivalenzen spezialisiert haben, hinsichtlich dieses Kriteriums bei der Fallgruppenzuordnung gezielt berücksichtigt werden. Auch sollte beachtet werden, dass ein entsprechender Case-Mix aus klar motivierten und ambivalent motivierten Patienten durchaus befruchtend sein kann, 
dass also nicht unbedingt von einer völligen Separation der ambivalent motivierten Patienten ausgegangen werden sollte.

Bei der Identifikation des Therapiewiderstandes finden sich die wichtigsten Unterscheidungskriterien zwischen ambivalenten Verhalten bei grundsätzlicher Motivation aber Ambivalenzkonflikt einerseits und Widerstand gegen eine Therapie andererseits wie folgt:

- „Aufsuchen der Therapieeinrichtung nur auf Drängen der Angehörigen,

- häufiges Zuspätkommen bzw. häufiges Absagen von Sitzungen,

- wiederholte Entscheidungen und Handlungen entgegen Absprachen mit dem Therapeuten,

- nichtdurchführen von Hausaufgaben,

- Rückschritte, wenn Erprobung neuen Verhaltens anstünde,

- Therapieabbruch ohne erkennbaren Anlass,

- unkooperatives oder aggressives Verhalten,

- unnötige Auseinandersetzungen mit dem Therapeuten,

- unangemessene Forderungen an den Therapeuten,

- Kritik am Vorgehen des Therapeuten,

- häufige Verwendung von - ich weiß nicht -,

- abrupter Themenwechsel oder häufige Missverständnisse mit dem Therapeuten."

(Grosse Holtforth und Michalak 2009, Seite 635).

Diese Verhaltensweisen finden sich speziell bei suchtkranken Patienten schon sehr häufig im Vorfeld, d. h. in der Auseinandersetzung mit der Suchtberatungsstelle, mit Hausärzten, mit behandelnden Ärzten auf Entgiftungsstationen und mit Mitarbeitern des Sozialdienstes bei Antragstellung. Neben den Möglichkeiten von Widerstand gegen die Therapie oder innerem Ambivalenzkonflikt mit gelebtem äußerlichen Ambivalenzverhalten, finden sich die hier beschriebenen Elemente auch noch bei Patienten mit starker Selbstunsicherheit und hohem Defizit im Bereich sozialer Kompetenz.

Das bemerkenswerteste Ergebnis dieser Untersuchung ergibt sich, aus Sicht des Autors, aus der Betrachtung der Gruppe der ambivalent motivierten Patienten. 
Es bestätigt sich erneut, dass gut motivierte, oder besser ausgedrückt eigenständig motivierte, Patienten ein deutlich besseres therapeutisches Outcome zeigen als ambivalent motivierte oder fremd motivierte Patienten. Zudem wird sichtbar, dass schlecht oder fremd motivierte Patienten ein besseres Therapieergebnis aufweisen als ambivalent motivierte.

Die ambivalent motivierten Patienten bilden also den negativen Scheitelbereich einer Parabel in einem Koordinationssystem mit den Hauptachsen Motivationslage und Therapieerfolg.

Es muss demnach davon ausgegangen werden, dass sich der Faktor „ambivalente Motivation“ maximal negativ auf den Therapieerfolg auswirkt. Bei genauerer Betrachtung des Phänomens wird dies nachvollziehbar. Der ambivalent motivierte Patient verliert kontinuierlich Kraft und Entscheidungsfähigkeit durch Nichtfestlegung. Die Zeit, in unserem Falle zerfließende Therapiezeit, arbeitet gleichsam gegen ihn.

Sowohl das Erleben des Patienten als auch des Therapeuten wird zunehmend von Frustration bestimmt.

Die Chance einer vorzeitigen Entlassung gegen ärztlichen Rat oder auf ärztlich therapeutische Anordnung steigt. In einigen Fällen wird der ambivalent motivierte Patient Fakten schaffen, die eine Entlassung erzwingen, dann könnte dies zur vorzeitigen, disziplinarische Entlassung führen.

Vermutlich dürfte aber der häufigste Ablauf beim ambivalent motivierten Patienten der sein, dass dieser seine Therapie regulär abschließt, ohne nennenswerte Veränderungen getätigt zu haben. Die Therapie wäre in diesem Falle von viel frustrierter therapeutischer Aktivität und wenig therapeutischem Ergebnis gefüllt.

Es kann davon ausgegangen werden, dass in einem Rehabilitationsrahmen, der auf Veränderung, Schulung, Erweiterung der inneren Ordnung und Entscheidungsbildung mit klarer Festlegung ausgelegt ist, ein ambivalent motivierter Patient zwangsläufig hinsichtlich Intensität und Tempo der Veränderung überfordert ist. Die hieraus resultierende Spannung wird er im Regelfall mit emotionaler Labilisierung beantworten. Es verbleiben ihm dann noch geringere Ressourcen um der Ambivalenzhaltung entgegen zu wirken. Rückkehr in alte Verhaltensmuster, bzw. Festigung derselben und damit erneute Stabilisierung der Ambivalenzproblematik ist die Folge. Dementsprechend macht es im Therapieergebnis und infolgedessen hinsichtlich der Abstinenzdauer kaum einen Unterschied, ob die Entlassform vorzeitig oder regulär sein wird. Innerhalb 
des begrenzten Therapiezeitraums und bei ergebnisorientierter Therapie, wird das eigentliche Therapieziel der Abstinenzsicherung langfristig nicht erreicht werden. Der stoffliche Rückfall bildet die logische Konsequenz und zwar trotz regulär abgeschlossener Therapie, durchaus im Stand der Arbeitsfähigkeit. D. h. laut Definition bei erfolgreichem Therapieergebnis.

Grundsätzlich müsste eine besondere Therapiestruktur konzipiert werden, die dem ambivalent motivierten Patienten zu klarerer Entscheidungsfähigkeit verhilft. Dies könnte dann eine deutlich längere Therapiedauer oder mehrere Therapieanläufe mit kurzer Dauer zur Folge haben. Die andere Möglichkeit wäre, dass die ambivalent motivierten Patienten durch Voruntersuchungen herausgefiltert werden, und die Therapie erst dann stattfindet, wenn der Ambivalenzkonflikt im Patienten nicht mehr stabil ist, sondern schon einer Lösung zugeführt werden kann. Wenn ein solches Assessment konzipiert und in die Fallgruppenbildung und damit in die Rehabilitationsteuerung eingebaut werden könnte, könnte von einem Effizienzsprung der Rehabilitationsbehandlung und somit einer Verbesserung des Therapieerfolgs von beachtlicher Größenordnung ausgegangen werden. Angesichts der nach wie vor hohen Nichtantrittsquote bei bereits kostengenehmigter Langzeitentwöhnungsmaßnahme, also ebenfalls einer motivationalen Problematik, sollte zudem die Frage des optimalen Ansatzpunktes für das informative Erreichen Alkoholabhängiger in der Gesellschaft und ihrer Zufuhr ins helfende System weiter erörtert werden.

Die derzeit in der aktuellen Fallgruppendiskussion beforschten Bereiche scheinen vor allem im Feld der Zugangssteuerung Verbesserungen erzielen zu wollen. Nach den hier vorliegenden Ergebnissen spielen die dort zu Grunde gelegten maßgeblichen Faktoren aber keine nennenswerte Rolle hinsichtlich des Therapieoutcomes und auch nicht in der 1-Jahres-Katamnese.

\section{zu 2)}

Die Frage, ob bestimmte Zugangsvariablen und insbesondere die Zugangsform zur Rehabilitationseinrichtung bei der Behandlung von Alkoholabhängigen eine Rolle hinsichtlich des Behandlungsergebnisses spielen, kann gemäß der hiesigen Ergebnisse mit ja beantwortet werden. Hierbei sind einige signifikante Zusammenhänge zwischen Zugangs- und Outcomevariablen sehr gut zu erklären, andere finden sich im Bereich der Deutung. Die Zusammenhänge sollen hier noch 
einmal im einzelnen beleuchtet werden. Die Motivationslage wurde bereits erörtert, sie wird hier nur noch marginal mit betrachtet.

Bezüglich der „Entlassform“:

- weist das Merkmal „Alter bei Aufnahme“ eine verminderte Wahrscheinlichkeit für eine reguläre Entlassung für älteren Patienten auf. Dieser Umstand steht zunächst gegen die Erwartung, da junge Patienten in Berufung auf ihre rasch mobilisierbaren Ressourcen eher die Therapie vorzeitig beenden, da sie sich zu stabil einschätzen und da ohnehin noch eine defizitäre Krankheitseinsicht vorliegt. Auf der anderen Seite sind ältere Patienten oft nicht mehr kritikfähig, weisen geringere Veränderungsbereitschaft auf und sind näher an einer Berentung oder bilanziert mutlos.

- weisen die Merkmale „Dauer Arbeitslosigkeit: 1 - 12 Monate“ und „Dauer Arbeitslosigkeit größer 12 Monate" eine verminderte Wahrscheinlichkeit für eine reguläre Entlassung auf. Dieser Umstand steht vermutlich im Zusammenhang mit der grundsätzlichen Perspektivlosigkeit bei vorausgegangener Arbeitslosigkeit, verlorengegangenen Handlungsalternativen und depressivem Empfinden bei lang andauernder Frustration. Bei teilweise desolatem Arbeitsmarkt, kommt es auch während der therapeutischen Maßnahme zu Resignation und Aufgabe.

- weisen die Merkmale „Erwerbssituation erwerbslos“ und „Erwerbssituation sonstige" eine erhöhte Wahrscheinlichkeit für eine reguläre Entlassung auf. Dieser Umstand steht zunächst gegen die Erwartung, da Erwerbslosigkeit und Arbeitslosigkeit gleichsinnig in der Wirkung erwartet wurde. Allerdings kann hinter dem Begriff der „Erwerbssituation erwerbslos“ beziehungsweise der „Erwerbssituation sonstiges“ auch eine zeitnahe betriebliche Kündigung aus Suchtgründen mit Option einer Wiederherstellung des Erwerbsverhältnisses oder auch eine berufliche Rehabilitation oder sonstige Maßnahme zur Förderung der Teilhabe am Arbeitsleben stehen.

- weist das Merkmal „anderer Schulabschluss“ eine verminderte Wahrscheinlichkeit für eine reguläre Entlassung auf. Dieser Umstand steht möglicherweise mit der Tatsache im Zusammenhang, dass gesonderte Abläufe in einem hochstrukturierten Therapiesystem immer besondere 
Mühen, Wege und Formalien bedingen, die dann eine Überforderung für den Betreffenden bedingen können.

- weisen die Merkmale „Familienstand ledig“ und „Familienstand geschieden“ eine verminderte Wahrscheinlichkeit für eine reguläre Entlassung auf, dies dürfte mit der Tatsache zusammenhängen, dass bestehende partnerschaftliche und familiäre Bindungen sowohl Anreiz als auch durch Verlustangst bedingten Druck erschaffen, eine Therapie regulär abzuschließen.

- weist das Merkmal „Alkoholfolgediagnose“ eine vermehrte Wahrscheinlichkeit für eine reguläre Entlassung auf. Dieser Umstand dürfte der Tatsache geschuldet sein, dass Patienten Zuwendung im somatischen Feld besser als Hilfe erkennen und günstige Entwicklungen dankbarer wertschätzen. Das heißt sie bewerten den Klinikaufenthalt an sich positiver, als Patienten ohne Folgeschäden. Zudem kann durch somatische Defizite eine erhöhte Krankheitseinsicht und damit bessere Motivation die Therapie regulär und erfolgreich abzuschließen vermutet werden.

Bezüglich des „Therapieergebnisses“:

- weist das Merkmal „weibliches Geschlecht“ eine verminderte Wahrscheinlichkeit für ein positives Resultat auf. Dieses Ergebnis deckt sich mit der Beobachtung, dass Frauen eine höhere Scham und ein ausgeprägteres Rückzugsverhalten aufweisen. Trotz höherer emotionaler Öffnungsbereitschaft könnte es also sein, dass verborgene Bereiche unterschiedlichen Ausmaßes unbearbeitet bleiben.

- weist das Merkmal „Abhängigkeitsdauer" eine verminderte Wahrscheinlichkeit für ein günstiges Therapieergebnis auf. Dies ist im Sinne der Chronifizierung, der Krankheit und der Verhaltensmuster mit erschwerter Möglichkeit zur Veränderung zu verstehen.

Bezüglich des „allgemeinen Leistungsbildes“:

- weist das Merkmal „Verbalgedächtnis“ eine erhöhte Wahrscheinlichkeit für eine gute Leistungsfähigkeit auf. Da im Leistungsprofil neben körperlicher 
und psychischer Leistungsfähigkeit die kognitiven Fähigkeiten beurteilt werden, kann hier von einer direkten Korrelation ausgegangen werden.

- weist das Merkmal „Erwerbssituation berentet“ eine verminderte Wahrscheinlichkeit für ein günstiges Leistungsbild bei Entlassung auf. Da im Falle der Altersberentung oder der dauerhaften Berentung kein Leistungsbild mehr erstellt werden muss beziehungsweise auch bei zeitlich begrenzten Berentungen im Vorfeld bereits ein Leistungsbild erstellt wurde, welches eine Erwerbsfähigkeit unter drei Stunden für den allgemeinen Arbeitsmarkt ausweist, ergibt sich hier auf alle Fälle eine deutlich leistungsgeminderte Vorauswahl.

- weist das Merkmal "Erwerbssituation sonstiges“ eine verminderte Wahrscheinlichkeit für ein hohes Leistungsprofil bei Entlassung auf. Eine unklare Erwerbssituation vor der Therapie könnte schon mit der Erfahrung einer verminderten Leistungsfähigkeit verbunden gewesen sein, was damit ebenfalls eine leistungsgeminderte Vorauswahl darstellt.

Bezüglich der „Abstinenz“:

- weist lediglich das Merkmal „ambivalente Motivation“ einen signifikanten Zusammenhang auf. Diese bedingt eine verminderte Wahrscheinlichkeit für das Erreichen einer abstinenten Lebensführung (siehe oben). Alle weiteren geprüften Zugangsvariablen zeigten hinsichtlich der Variablen Abstinenz, keine signifikanten Zusammenhänge.

Bezüglich „Erwerbssituation ein Jahr nach Entlassung“:

- weist das Merkmal "Wartezeit auf eine Therapie“ eine verminderte Wahrscheinlichkeit für die „Erwerbstätigkeit ein Jahr nach Entlassung“ auf. Dieser Umstand dürfte der Tatsache geschuldet sein, dass Wartezeiten per se zermürbend und im speziellen Falle, $d . h$. bei Warten auf die helfende Maßnahme, teilweise vital belastend erlebt werden. Zudem kommt es in den Warteepisoden häufig zu Rückfällen, depressiven Stimmungslagen, Konfliktsituationen etc., die mit insuffizientem Handlungsinventar durchgestanden werden müssen. Die Motivation dürfte in einem hohem $\mathrm{Maße}$ labilisiert werden, je länger die Wartezeit andauert. Um so stärker ist dann von einer Entwicklung in Richtung Mutlosigkeit und Selbstaufgabe auszugehen. Diese Interpretation deckt sich auch mit dem Phänomen, dass 
die „Ambivalenzmotivation“ hinsichtlich des Merkmals „Erwerbsfähigkeit nach einem Jahr" einen gleichsinnigen, signifikanten Zusammenhang wie die Wartezeit aufweist.

- weisen die Merkmale „verbale Intelligenz“ und „Verbalgedächtnis“ eine erhöhte Wahrscheinlichkeit für die „Erwerbsfähigkeit nach einem Jahr“ auf. Da die „verbale Intelligenz" und das "Verbalgedächtnis“ kognitive Fähigkeiten darstellen, die in allen Kommunikations- und Arbeitszusammenhängen benötigt werden, können sie als Voraussetzungsfaktoren für das betrachtete Merkmal gesehen werden.

- weist das Merkmal „Abhängigkeitsdauer" eine verminderte Wahrscheinlichkeit für das Zustandekommen eines Tätigkeitsverhältnisses nach einem Jahr auf. Dieser Umstand ist mit der Problemchronifizierung (siehe oben), die mit der Abhängigkeitsdauer direkt korreliert, hinreichend erklärt.

- weist das Merkmal "Dauer der Arbeitslosigkeit“, mit seinen Untergruppierungen „Arbeitslosigkeit 1-12 Monate“ und „Arbeitslosigkeit größer 12 Monate“, eine verminderte Wahrscheinlichkeit für das Zustandekommen eines Tätigkeitsverhältnisses nach einem Jahr auf. Auch hier kann der Umstand der Chronifizierung sowie der Gewohnheit und damit den erschwerten Bedingungen für eine Verhaltensänderung und einer notwendigen Korrektur der Lebensumständen, ausreichend klären, weshalb sich hier Hemmnisse für eine Reintegration ins erwerbstätige Leben finden.

- weist das Merkmal „Erwerbssituation erwerbslos“ eine erhöhte Wahrscheinlichkeit für eine Erwerbstätigkeit nach einem Jahr nach Entlassung auf. Dieser Umstand steht gegen die Erwartung, widerspricht sie doch den zuvor getätigten Interpretationen. Eventuell kann das Phänomen dadurch erklärt werden, dass für Menschen, die alkoholkrank und zum Zeitpunkt des Therapiebeginns erwerbslos sind, bereits seitens der öffentlichen Förderung mehr stützende Maßnahmen vorbereitetet sind, als für Gruppen von Menschen, die einen weniger offensichtlichen Unterstützungsbedarf signalisieren. „Erwerbssituation erwerbslos“ sagt hier nichts über die Dauer und damit auch nichts über die Chronifizierungsproplematik, die durch vorheriger Arbeitslosigkeit bedingt sein kann, aus. Auch Menschen, die zuvor vom Arbeitgeber gekündigt 
wurden mit Perspektive einer Wiederanstellung nach erfolgreicher Reha, sowie solche, denen von öffentlicher Hand eine berufliche Fördermaßnahme nach erfolgter Rehabilitationsmaßnahme zugesagt wurde, fallen unter diese Gruppe. Unter dem Merkmal „Erwerbssituation erwerbslos" könnte sich also ein motivationaler Aspekt für die Behandlungsaufnahme und die Therapie verbergen.

- weist das Merkmal "Sonderschulabschluss" eine erhöhte Wahrscheinlichkeit für eine positive Erwerbssituation nach einem Jahr auf. Für diesen Bereich gelten die selben Überlegungen wie im Punkt „Erwerbssituation erwerbslos“ (siehe oben) beschrieben, das heißt der Umstand, dass Förderprogramme bereits installiert sind, da die Gruppe der Alkoholabhängigen, die über einen Sonderschulabschluss verfügen, durch die Sozialorgane bereits besser im Blick sind, könnte zu einer überdurchschnittlichen Förderung führen.

- weist das Merkmal „Kostenträger LVA Thüringen“ im Vergleich zur Referenzkategorie „Kostenträger BfA“ eine erhöhte Wahrscheinlichkeit für eine positive Erwerbssituation nach einem Jahr auf. Dieser Umstand kann anhand der hier vorliegenden Datenlage nicht erklärt werden, eventuell sind hier regionale Umstände wie Bauvorhaben oder Firmenförderung etc. verantwortlich.

- weist das Merkmal „Familienstand“ und zwar sowohl „Familienstand ledig“ und „Familienstand geschieden“ eine erhöhte Wahrscheinlichkeit für eine günstige Erwerbssituation ein Jahr nach Entlassung auf. Das Merkmal ist während der Therapie (das heißt für die reguläre Entlassform) mit verminderter Wahrscheinlichkeit belegt, für den posttherapeutischen Abschnitt aber als günstig zu sehen. Vermutlich dürfte dies mit Mobilität, klar getroffenen Entscheidungen, Abgrenzungen und Neuanfang erklärt sein.

- weisen die Merkmale „psychische Zusatzdiagnose“ und „Persönlichkeitsstörung“ eine höhere Wahrscheinlichkeit für eine günstige Erwerbssituation nach einem Jahr auf. Auch hier ergibt sich ein Ergebnis, welches zunächst gegen die Erwartung steht. Vermutlich aber sind die beiden Merkmale zwar während einer Therapie eher hinderlich (siehe oben), nach abgeschlossener, erfolgreicher Therapie könnten dagegen verbesserte Förderprogramme und, vor allem bei der 
Persönlichkeitsstörung, eine hohe Rigidität und damit Beharrlichkeit beim Erreichen von Zielen eine Rolle spielen, welche die Phänomene eventuell erklären.

Zusammenfassend kann gesagt werden, dass bei der Betrachtung des Zugangswegs zur Therapieeinrichtung, vor allem also der Variablen „Auflage“, kein signifikanter Zusammenhang mit den untersuchten Outcomevariablen gefunden wurde. Das bedeutet, dass die vom Inhalt losgelöste Organisationsform, die einen alkoholabhängigen Patienten in die behandelnde Einrichtung steuert, laut dieser Untersuchung keine Bedeutung hat. Einzelne der hier überprüften Zugangsvariablen spielen im sozialmedizinischen Bereich eine Rolle, im Bereich des Therapieergebnisses, welches auf die Abstinenz zielt, aber nicht.

zu 3)

Die patientenbezogenen Daten der Untersuchung bilden die zugangsrelevanten Merkmalsbereiche der RMK-Forschung in einigen Feldern ab, allerdings nicht vollständig. Bei beschriebenem, signifikanten Zusammenhang zwischen einzelnen Bereichen der testpsychologischen Aufnahmeuntersuchung der untersuchten Klientel zu späterer Erwerbstätigkeit (1-Jahres-Katamnese) ist beispielsweise zu erwarten, dass in den eingesetzten Assessments für die Rehabilitationsteuerung weitere positive Prädiktoren für eine erfolgreiche Therapie erfasst werden. Kritisch gesehen werden aber muss der Umstand, dass die Motivationslage, die in der vorliegenden Untersuchung mit Abstand den eindeutigsten prädiktorischen Faktor abbildet, in dem Bereich, in dem die RMK-Forschung mit den Fallgruppen ansetzt, nicht nennenswert zum Tragen kommt. Inwieweit durch die Filterfunktion der modernisierten und verbesserten Zugangssteuerung die Nicht-Antrittsquote gesenkt werden kann, kann hier nicht beurteilt werden, muss allerdings ebenfalls kritisch betrachtet werden. Aus Sicht des Autors stellt sich auf jeden Fall die Frage, inwieweit der Fokus der Rehabilitationsforschung für Abhängigkeitserkrankungen nicht verstärkt auf das Problem der Motivationsklärung vor der Therapie und innerhalb der Therapie ausgerichtet werden sollte.

An dieser Stelle sei auf ein regional beforschtes Zugangsverfahren, die Direktverlegung verwiesen (Retzlaff et al. 2009). Nach der Entgiftungsbehandlung wird der Patient direkt in die Entwöhnungsbehandlung übergeleitet. Wartezeiten, 
Stressexposition im alten Umfeld, Rückkehr in alte Verhaltensmuster, bis hin zum stofflichen Rückfall, können durch extern, strukturelle Sicherung besser vermieden werden. Eine wesentliche vulnerable Phase wird hierdurch überbrückt. In dieser werden vermutlich keine großen therapeutischen Erfolge erzielt, der vor sich selbst geschützte Patient hat aber eine bessere Voraussetzung seine Motivation zu klären. Außerdem ist damit eine Begleitung durch behandelndes Personal gegeben. Wenn ein Verbund, in dem eine "Direktverlegung" organisiert ist, dicht genug strukturiert ist, so dass Vorbegegnungen mit den Nachbehandlern und Nachbetreuung mit den Vorbehandlern möglich gemacht werden kann, könnten einzelne Patienten überlappend wie ein „Staffelstab“ übergeben werden. Eine höhere Kontinuität kann bei einer Verlegung eines Patienten in eine andere Behandlungseinheit kaum erreicht werden. Es ist zudem davon auszugehen, dass in einem solchen Übergabeverlauf die Quote der Therapie-Nichtantreter minimiert wird; schließlich sind äußere Motivationshemmnisse, vor allem Verführung und Alltagsbelastung, nahezu eliminiert.

In einer Untersuchung, wer von der Direktverlegung profitiert (Kemmann et al. 2009), zeigt der Autor auf, das bestimmte Bündel aus Zugangsvariablen, hier „Erwerbssituation“, „mittlerer Dauer vorheriger Arbeitslosigkeit“ und „Abhängigkeitsdauer", durchaus eine Bedeutung für das Behandlungsergebnis haben können. Dabei tritt das Phänomen einer Kombination von Zugangsvariablen bei den direktverlegten Patienten auf. Es bleibt offen, ob dieser Bündelung oder der Direktverlegung an sich die entscheidende Rolle für ein signifikant besseres Ergebnis zugeordnet werden muss. Es kann aber auf alle Fälle davon ausgegangen werden, dass die Regionalität, d. h. die Möglichkeit den Patienten vor Ort und damit auch nach klinischen Gesichtspunkten zu steuern, hinsichtlich des individuellen Behandlungs- und Betreuungsbedarfs ein deutlicher Vorteil gegenüber den groß angelegten Steuerinstrumenten wie Fallgruppen oder RMK darstellt.

Ein weiteres, bislang wenig bearbeitetes Feld bei den Untersuchungen der Rehabilitationszugangssteuerung findet sich im Bereich der körperlichen Komorbidität. Bei teilweise massiven körperlichen Beeinträchtigungen verändert sich der Gesamtrehabilitationsverlauf, die Belastbarkeit und das Maß der Einschränkungen eines Patienten in derart hohem Umfang, dass im Basissetting verankerte therapeutische Elemente zum Teil nicht zur Anwendung gelangen können. Bei der vorliegenden Untersuchung spielt diese körperliche Komorbidität 
als Prädiktor zwar keine Rolle, allerdings wurden die körperlichen Faktoren in der Untersuchung auch noch nicht dezidiert erfasst. Eine weitere Untersuchung für spätere Jahrgänge, mit der Fragestellung, welchen Effekt die zu Therapiebeginn vom Patienten benannten körperlichen Beeinträchtigungen auf das Therapieoutcome haben, wird im Diakonie-Krankenhaus Elbingerode durchgeführt und soll in Kürze veröffentlicht werden. $\mathrm{Ob}$ und in welchem Ausmaß dann Ergebnisse aus diesem Bereich in zukünftige Kategorien bei Fallgruppenbildung oder ähnlichen Klassifizierungen einfließen, ist sicher ein weiterer Forschungsgegenstand.

\subsection{Fazit für die Praxis}

1. Das Erfassen und Definieren von Prädiktoren für das Outcome einer Langzeitentwöhnungsbehandlung von Alkoholabhängigen gestaltet sich ausgesprochen schwierig. Krankheitsimmanente Paradoxien und Irrationalitäten sowie Phasen mit stark unterschiedlichem Betreuungsbedarf sind neben Verleugnungsmechanismen, Angst, Scham und Realitätsverlust ursächlich dafür zu sehen, dass die Datenerhebung der betroffenen Patienten immer in hohem Maße unscharf bleibt. Entsprechend sind wenig messbare und vergleichbare Parameter, die prädiktorisch verwendbar sind, abzuleiten.

2. Der Zugangsweg zur Klinik spielt eine untergeordnete Rolle. Entscheidend ist, dass der Betroffene die behandelnde Einrichtung überhaupt erreicht. Die Reduktion der Nichtantrittsquote muss ein vorrangiges Ziel der Qualitätsverbesserung bei der Behandlung von Suchtkranken sein.

3. Die Ergebnisse der Rehabilitationsforschung sind im Sinne einer Standardisierung und damit qualitativen Behandlungsangleichung der Rehabilitationseinrichtungen untereinander durchaus als sinnvoll anzusehen. Im Bereich Verbesserung der Therapiemotivation und Reduktion der NichtantrittsQuote dürften sich die Ergebnisse der Forschung wenig positiv auswirken. Grund hierfür ist der Umstand, dass auch eine sehr ausgefeilte Zugangssteuerung nicht denselben motivationalen Effekt erbringen kann, wie eine persönliche Begleitung und Betreuung auf dem Hintergrund der gewachsenen Beziehung. 
4. Die Therapiemotivation der Patienten stellt von allen überprüften Variablen mit Abstand den Hauptprädiktor für das therapeutische Outcome einer Langzeitentwöhnungsmaßnahme dar. Bei der Qualitätsverbesserung der Behandlungsketten sollten also vor allem die Bereiche gefördert werden, in denen die Therapiemotivation von Betroffenen gestärkt wird. Dies bedeutet Werbung, Annahme und individuelle, bedarfsgerechte Begleitung. Zu Beginn eines Weges aus der Sucht, finden sich stets Phasen, in denen die Notwendigkeit einer paternalistischen Betreuung besteht.

5. Das bemerkenswertestes Ergebnis der Arbeit ist das Faktum, dass die ambivalent motivierten Patienten nicht nur, wie erwartet, ein schlechteres Therapieoutcome aufweisen als die eigenmotivierten Patienten, sondern auch als die primär fremdmotivierten Patienten. Der Zusammenhang zwischen wenig motiviert, teilmotiviert und motiviert ist im Bezug auf das Therapieergebnis also nicht linear, vielmehr zeigt sich im Bereich der ambivalent motivierten Patienten ein Tiefpunkt für den Therapieerfolg. Dies erklärt sich aus der Tatsache, dass ambivalent motivierte Patienten eine regulär abgeschlossene, d. h. mit maximalem Behandlungsaufwand gestaltete Therapie absolvieren können, ohne nennenswerte Veränderungen vollzogen oder maßgebliche Entscheidungen getroffen zu haben. Die fremdmotivierten und damit geringgradig eigenmotivierten Patienten dagegen werden gemäß ihrer Fähigkeit, eine Position zu beziehen, eher Fakten schaffen und damit Entscheidungen treffen. Die Therapieabbruchsrate ist zwar höher, als bei den ambivalent motivierten Patienten, die langfristige Erfolgsrate aber ebenfalls, da die Patienten, die fremdmotiviert waren und die gesamte Therapiedauer und das gesamte Therapieprogramm in Anspruch nahmen, im Bereich Krankheitseinsicht und Veränderungsbereitschaft stärker hinzu gewonnen haben. Der Wechsel aus der Position des fremdmotivierten Patienten in die des motivierten Patienten gelingt also leichter, als der aus der Position des ambivalent motivierten Patienten. 


\subsection{Methodenkritik}

Methodische Schwächen:

- Es handelt sich um eine retrospektive Studie, d. h. die Datenlage wurde nicht speziell für die Untersuchung erhoben.

- Teile der Daten mussten aus den vorliegenden Quellen abgeleitet werden, eine direkte Datenerfassung war nicht immer möglich.

- Der Datenpool ist auf einen Jahrgang behandelter alkoholabhängiger Patienten ( $N=434)$ einer einzelnen Klinik beschränkt und damit klein und nicht im Vergleich mit anderen Patientendaten. Es bleibt offen, inwieweit die hier gewonnenen Ergebnisse auf größere beispielsweise bundesweite Gegebenheiten übertragbar sind.

- Die Datenlage war zum Teil aufgrund einer lückenhaften Dokumentation und einer Rücklaufquote von etwas mehr als 50 \% unvollständig.

- Die Haupthypothese 3 wurde nicht statistisch überprüft. Stattdessen wurden aus den vorliegenden Ergebnissen induktive Schlussfolgerungen abgeleitet.

- Die Daten, die im Rahmen der katamnestischen Untersuchung erhoben wurden, sind nur in geschätzten Standards zu gewichten.

- Bei Suchterkrankungen ist die Gefahr einer unrealistisch positiven Antwort in den Katamnesebefragungen als überdurchschnittlich hoch zu erwarten.

- Die Datenerhebung sowie die vorliegende Arbeit wurde von Behandlern der Klinik durchgeführt. Die Gefahr subjektiver Interpretation von Ergebnissen ist erhöht. 
Methodische Stärken:

- Dass die Datenerhebung sowie die vorliegende Arbeit von Behandlern der Klinik durchgeführt wurde, ist aber auch als Stärke zu sehen. Der klinische Bezug zum Thema blieb durchgehend vorhanden. Persönliche Beziehungen zur Untersuchten validierten z. T. die Daten.

- Durch die Regionalität und das Vorhandensein eines Suchtverbundes war die Weiterbetreuung und Beobachtung der behandelten Patienten über längere Zeiträume möglich. 


\section{Zusammenfassung}

Auf dem Hintergrund der grundsätzlichen Frage nach Wirkfaktoren bei der Rehabilitation Suchtkranker, die ggf. prädiktorische Aussagekraft für eine Therapie besitzen, wurden Zugangswege und Zugangsvariablen zur Rehabilitationseinrichtung sowie die Motivationslage von Patienten untersucht. Die Forschungsergebnisse der Rehabilitationsforschung, die in diesem Bereich Untersuchungen durchführt (Fallgruppen, Rehabilitanden-ManagementKategorien), wurden beleuchtet.

Die Patientengruppe des Entlassjahrgangs 2004 mit der Hauptdiagnose Alkoholabhängigkeit der medizinischen Rehabilitation Sucht des DiakonieKrankenhauses Harz $\mathrm{GmbH}$ in Elbingerode wurde untersucht. Differenziert wurde zunächst nach den Zugangswegen. Eine teilweise davon abgeleitete Unterscheidung nach intrinsischer und extrinsischer Motivation fand statt. Eine dritte Patientengruppe mit „ambivalenter Motivationslage“ wurde gesondert betrachtet. Outcomevariablen waren Therapieergebnis, Entlassform und Leistungsprofil zum Entlasszeitpunkt sowie Erwerbssituation und Suchtmittelabstinenz nach einem Jahr. Die erhobenen Zahlen wurden statistisch mit multiplen Regressionsverfahren analysiert.

Ein Zusammenhang zwischen Zugangsweg bzw. Art der Zugangssteuerung und Abstinenzrate konnte nicht hergestellt werden.

Es fanden sich jedoch Zusammenhänge zwischen Motivationslage und Entlassform, Therapieergebnis und Abstinenzrate.

Die bekannten Unterschiede zwischen selbstmotivierten und fremdmotivierten Patienten (Auflagen) konnten bestätigt werden. Auffällig war, dass bei allen Patienten, die eine klare Haltung und Position zu ihrer Erkrankung aufwiesen (unabhängig ob Krankheitseinsicht oder Krankheitsabwehr vorlag), ein signifikant höherer Behandlungserfolg gegenüber den ambivalent positionierten Patienten ausgewiesen werden konnte.

Einigen der untersuchten Zugangsvariablen konnte eine prädiktorische Aussagekraft für das Therapieergebnis zugeschrieben werden, eine hohe Übereinstimmung zu den aktuell verwanden Kriterien in den Rehabilitanden- 
Management-Kategorien fand sich nicht. In einzelnen Bereichen der testpsychologischen Diagnostik konnten Faktoren gefunden werden, die als Zugangsvariablen eventuell für die weitere Assessmententwicklung wertvoll sein könnten.

Psychische und körperliche Komorbiditäten scheinen von untergeordneter Bedeutung bei der Abstinenzrate zu sein, obwohl sie den Zugangsweg in hohem Maße bedingen. Bezüglich der sozialmedizinischen Fragestellung, wie Leistungsbild und Erwerbssituation nach einem Jahr, spielen diese Komorbiditäten neben der Erwerbssituation vor der Rehabilitation offensichtlich eine bedeutendere Rolle. Es sollte geprüft werden, ob die Hinzunahme des Faktors somatische Komorbidität bei der Fallgruppenbildung die Effizienz der bisherigen Forschungsergebnisse weiter vertiefen kann.

Inwieweit die übergeordnete Rehabilitationssteuerung Einfluss auf die Motivationslage nehmen und in Folge beispielsweise das große Problem der hohen Nichtantrittsquote bei genehmigter Therapie beeinflussen kann, bleibt offen. Vermutlich aber haben regionale Steuerungsmodalitäten, wie sie in einem Suchtverbund gegeben sind, eine höhere Chance die Motivation zu prüfen und sinnvoll darauf einzugehen. 


\section{Literaturverzeichnis}

1. Beckmann U, Grünbeck P, Naumann B, Klosterhuis H: Aktuelle Ergebnisse aus der Qualitätssicherung der Suchtrehabilitation. Suchtaktuell 16: 29 - 34 (2009)

2. Deutsche Gesellschaft für Suchtforschung und Suchttherapie e. V. (Hrsg.): Dokumentationsstandards III für die Evaluation der Behandlung von Abhängigen. Sucht 47: 89 - 90 (2001)

3. Deutsche Rentenversicherung Bund (Hrsg.): Der ärztliche Rehabilitationsentlassungsbericht - Leitfaden zum einheitlichen Entlassungsbericht in der medizinischen Rehabilitation der gesetzlichen Rentenversicherung, Berlin, S. 25 - 55 (2009)

4. Egner U, Spyra K, Vorsatz N: Suchtbehandlung passgenau! - Anforderungen und Entwicklungspotentiale aus Sicht der Deutschen Rentenversicherung Bund. Suchtaktuell 16: $21-25$ (2009)

5. Eid M, Gollwitzer M, Schmitt M: Multiple Regressionsanalyse. In: Eid M, Gollwitzer M, Schmitt M (Hrsg.) Statistik und Forschungsmethoden, Beltz, Weinheim, Basel, S. $602-698$ (2010)

6. Eid M, Gollwitzer M, Schmitt M: Logistische Regressionsanalyse. In: Eid M, Gollwitzer M, Schmitt M (Hrsg.) Statistik und Forschungsmethoden, Beltz, Weinheim, Basel, S. $767-810$ (2010)

7. Fachausschuss Sucht des AHG-Wissenschaftsrates (Hrsg.): Erhebungsbogen der Basisdokumentation Sucht. Version 1.2. Hilden (2002) In: Basisdokumentation BADO Sucht PFW - Elektronisches Handbuch des PatfakPatientenverwaltungsprogramms, Redline Data GmbH, Ahrensbök, S. 1 - 7 (Stand 31.12.2004) 
8. Fachausschuss Sucht des AHG-Wissenschaftsrates (Hrsg.): Nachbefragungsbogen der Katamnese Sucht. Version 1.0. Hilden (2002) In: Basisdokumentation BADO Sucht PFW - Elektronisches Handbuch des PatfakPatientenverwaltungsprogramms, Redline Data GmbH, Ahrensbök, S. 8 - 12 (Stand 31.12.2004)

9. Feuerlein W: Alkoholismus. Warnsignale - Vorbeugung - Therapie. Bedingungsgefüge des Alkoholismus, C. H. Beck, München, S. 18 - 19 (2008)

10.Funke W: Suchtbehandlung passgenau!? Entwicklungsmöglichkeiten und Anforderungen aus Sicht der Behandler - Ein Thesenpapier. Suchtaktuell 16: $26-28$ (2009)

11. Geyer D, Batra A, Beutel M, Funke W, Görlich P, Günthner A, Hutschenreuter $U$, Küfner $H$, Möllmann $C$, Müller-Fahrnow W, Müller-Mohnssen M, Soyka M, Spyra K, Stetter F, Veltrup C, Wiesbeck G A, Schmidt L G: AWMF-Leitlinie Postakut Behandlung alkoholbezogener Störungen. Sucht 52: 8 - 34 (2006)

12. Grawe K: Neuropsychotherapie. Schlussfolgerungen für die Psychotherapie, Hogrefe, Göttingen, S. $372-440$ (2004)

13. Grosse Holtforth M, Michalak J: Spezielle Indikationen. Bearbeitung von Ambivalenzen. In: Margraf J, Schneider S (Hrsg.) Lehrbuch der Verhaltenstherapie, Bd 2, Störungen im Erwachsenenalter, Springer, Berlin, S. $632-646$ (2009)

14. Hänsgen K-D, Rosenfeldt B: Verbalgedächtnistest (VEG). Elektronisches Handbuch. In: Hogrefe Test-System 3.8.4. Hogrefe, Göttingen, ohne Seitenaufteilung (2005)

15. Keck T, Hansmeier T, Haaf H G: Patientenfallgruppen in der medizinischen Rehabilitation aus Sicht der Rentenversicherungsträger. Praxis, klinische Verhaltensmedizin und Rehabilitation 21: 99 - 103 (2008) 
16. Kemmann D, Gänsicke M, Forschner L, Miehe K: Neue Zugangswege zur Entwöhnungsbehandlung - Erfahrungsberichte aus 4 Kliniken. Suchtaktuell 16: $51-61$ (2009)

17.König H-H: Fallgruppen aus gesundheitsökonomischer Perspektive. Praxis, klinische Verhaltensmedizin und Rehabilitation 21: 85 - 91 (2008)

18. Kuhl J, Kazén M: Persönlichkeits-Stil- und- Störungs-Inventar (PSSI), Hogrefe, Göttingen, S. 7 - 18 (1997)

19. Kulick B: Innovative Elemente der Entwöhnungsbehandlung. Suchtaktuell 16: 5 - 11 (2009)

20. Lindenmeyer J: Patientenfallgruppen in der medizinischen Rehabilitation am Beispiel der Rehabilitation von Alkoholkranken. Praxis, klinische Verhaltensmedizin und Rehabilitation 21: 135 - 142 (2008)

21. Missel P, Bachmeier R, Funke W, Garbe D, Herder F, Kersting S, Medenwaldt J, Schneider B, Verstege R, Weissinger V, Wüst G: Effektivität der stationären Suchtrehabilitation - FVS-Katamnese des Entlassjahrgangs 2008 von Fachkliniken für Alkohol- und Medikamentenabhängige. Suchtaktuell 1: 15 - 26 (2011)

22. Pfeiffer-Gerschel T, Steppan M, Hildebrand A, Wegmann L: Jahresstatistik der professionellen Suchtkrankenhilfe. In: Deutsche Hauptstelle für Suchtfragen e. V. (Hrsg.) Jahrbuch Sucht 2010, Neuland, Geesthacht, S. 165 - 188 (2010)

23. Prochaska J O, DiClemente C C: The transtheoretical approach. In: Norcross J C, Goldfried, M R (Hrsg.) Handbook of psychotherapy integration, Oxford, New York, S. 147 - 171 (2005)

24.Rapp B: Konzepte der Fallgruppenbildung im Akutsektor und im Rehabilitationsbereich. Praxis, klinische Verhaltensmedizin und Rehabilitation 21: $92-97$ (2008) 
25. Retzlaff R, Hildebrandt M, Bechmann M, Ueberschär I: Neue Zugangswege zur Entwöhnungsbehandlung in Sachsen, Sachsen-Anhalt und Thüringen Rehabilitation effizient und rentabel. Suchtaktuell 16: 43 - 51 (2009)

26. Rische H: Neues Instrument der Rehabilitationsqualitätssicherung eingeführt Rehabilitationstherapiestandards Alkoholabhängigkeit. Reha-QS Newsletter 2 (2009) in: http://www.deutsche-rentenversicherung-bund.de/SharedDocs/de/ Funktionen/Newsletter/GenerierteNewsletter/2009-12-10 RehaQualitaetssicherung Newsletter 13:45:04.html?nn=154946 (Stand: 11.04.2012)

27. Scheibler F, Janssen C, Pfaff: Shared Decision Making: ein Überblicksartikel über die internationale Forschungsliteratur. Sozial- und Präventivmedizin 48: $11-24$ (2003)

28. Schmidt K-H, Metzler P: Wortschatztest (WST), Beltz, Weinheim, S. $3-11$ (1992)

29. Soyka M, Schmidt P, Bottlender M: Wissenschaftliches Projekt: Rehabilitationstherapiestandards Alkoholabhängigkeit - Pilotversion. Deutsche Rentenversicherung Bund (Hrsg.) Berlin, S. 4 - 29 (2009)

30.Spyra K, Ammelburg N, Köhn S: Rehabilitanden-Management-Kategorien (RMK): Überblick zu den Ergebnissen aus der bisherigen Forschungs- und Entwicklungsarbeit. Praxis, klinische Verhaltensmedizin und Rehabilitation 23: $63-80(2010)$

31. Vogelsang M: Die Behandlungspraxis zwischen Individualisierung und Standardisierung - unter besonderer Berücksichtigung der Komorbidität. Suchtaktuell 16: 12 - 15 (2009)

32. Wanke K: Normal - abhängig - süchtig: Zur Klärung des Suchtbegriffs. In: Deutsche Hauptstelle gegen die Suchtgefahren (Hrsg.) Süchtiges Verhalten: Grenzen und Grauzonen im Alltag, Hoheneck, Hamm, S. 11-22 (1985) 
33. Weidenhammer W, Fischer B: c.l.-Skala, ein Selbstbeurteilungsverfahren zur Objektivierung leichter cerebraler Insuffizienzen, Vless, Ebersberg, S. 7 - 22 (1987) 


\section{Anhang 1}

\section{christliches Krankenhausleitbild}

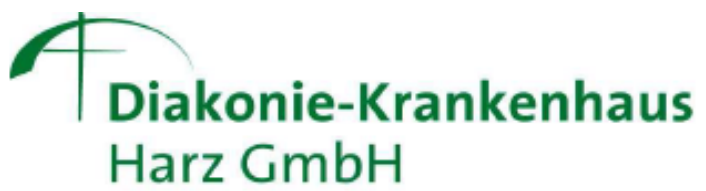

\section{Krankenhausleitbild}

Die Diakonie-Krankenhaus Harz GmbH, als Teil des Deutschen GemeinschaftsDiakonieverbandes, wendet sich an Menschen mit den unterschiedlichsten Erkrankungen und gibt ihnen fachlich kompetente und christlich engagierte Hilfe.

Dazu bieten wir eine multiprofessionelle Krankenhausbehandlung in unseren medizinischen Fachgebieten an. Als evangelisches Krankenhaus sind uns geistliche, seelsorgerliche und soziale Angebote wichtig.

Dabei ist die Grundlage unseres Handelns das Wissen darum, dass jeder Mensch von Gott gewollt und geliebt, einmalig und unverwechselbar ist.

Daraus resultiert:

im Hinblick auf die Patienten

- Wir schätzen unsere Patienten mit ihrer individuellen Biographie und begegnen ihnen mit aufrichtiger Achtung.

- Wir bieten unseren Patienten fachlich-qualifizierte und umfassend moderne medizinische und therapeutische Behandlung an. Maßstab für deren Qualität ist kurzfristige Hilfe, mittelfristige Stabilisierung und langfristige Zufriedenheit entsprechend dem jeweiligen Krankheitsbild.

- Wir bieten den Patienten Orientierung an, in dem wir in Reden und Handeln auf die Gute Nachricht von Jesus Christus hinweisen.

im Hinblick auf die Mitarbeitenden

- Wir arbeiten gemeinsam an der Entwicklung und Festigung der Identifikation jedes einzelnen Mitarbeitenden mit unseren Krankenhäusern und dem DGD insbesondere dem Diakonissen-Mutterhaus „Neuvandsburg"

- Wir erweitern unsere fachlichen und zwischenmenschlichen Beziehungen.

- Wir streben eine fortwährende Qualitätsverbesserung an.

im Hinblick auf die Einrichtung und die Öffentlichkeit

- Wir kommunizieren, dass auch der kranke Mensch Anrecht auf ein menschenwürdiges Leben hat.

- Wir suchen die Kooperation mit allen, die sich in den Therapieprozess unserer Patienten mit einbringen (Ärzte und Krankenhäuser, wissenschaftliche Forschungsund Ausbildungseinrichtungen, Beratungs- und Betreuungsstellen, Selbsthilfegruppen und der Hospizbewegung, christliche Gemeinden und Ehrenamtliche).

- Wir wollen in der Öffentlichkeit erkennbar sein und bemühen uns deshalb um Transparenz, z. B. durch Qualitätssicherungsmaßnahmen und regelmäßige Information der Öffentlichkeit.

- Wir gehen mit den Ressourcen unserer Einrichtung und Umwelt verantwortlich und wirtschaftlich um.

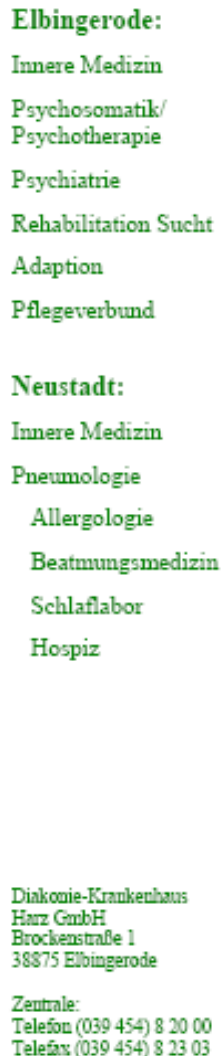




\section{Anhang 2}

$\underline{\text { Aufzählung Stammdaten }}$

- $\quad$ Alter aktuelle (am Auswertungstag)

- $\quad$ Alter bei Aufnahme in Jahren

- $\quad$ Alter bei Entlassung (bei nicht Entlassenen = aktuell)

- Aufenthaltsdauer in Tagen bei bereits Entlassenen

- $\quad$ Aufenthaltstage im ausgewählten Zeitraum

- $\quad$ Aufnahmedatum

- Aufnahmenummer

- Entlassungsdatum

- $\quad$ Geschlecht

- Hauptleistungsträger

- HIV-Code nach ohltec Horizont-Berechnung

- $\quad$ HIV-Code nach ZIS-Hamburg-Berechnung (BaDo HH)

- $\quad$ Name des Patienten

- $\quad$ Nationalität

- Ort

- Postleitzahl

- Therapiegruppe

- Vorname

- Zeit von Kostenzusage bis Aufnahme in Tagen

- Zimmer 


\section{Anhang 3}

Basisdokumentation Sucht (AHG - Wissenschaftsrat, 2002 - Programmversion 1.2; schriftliche Form übernommen aus dem Patfak für Windows - Handbuch, Stand 31.12.2004)

Klinische Basisdokumentation PFW VI.2

In Anlehnung an de BADO Suche

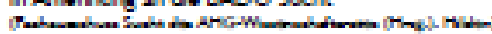

eKIriken Wiad CmbH \&. Co KG und Radine DATA GmbH

\section{Eingangsphase}

211 Wiederaufnahme in die Klinilk

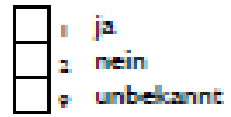

101 Familiensituation bei Betreuungsbeginn

$\begin{array}{ll}\square & \text { ledig } \\ 2 & \text { verheiratet, zusammenlebend } \\ 2 & \text { verheiratet, getrennt lebend } \\ 4 & \text { verwitwet } \\ \text { 2 geschieden } & \text { unbelcannt }\end{array}$

103 zeitlich überwiegende Lebenssituation

in den leczten 6 Monaten
1 alleinlebend
2 mit Etternteil
4 mit Kindern
5 mit Partner(in)
o mit Freunden/Bekannten
7 mit sonstigen Personen
onbekannt

105 Höchster bisher erreichter Schulabschluss

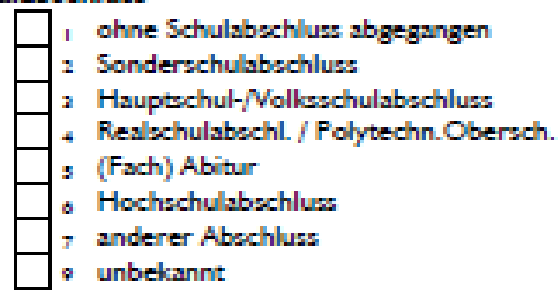

107 Lecater beruflicher Status

\begin{tabular}{|c|c|}
\hline 01 & Arbeiter \\
\hline 02 & Facharbeiter, Lernberuf \\
\hline or & $\begin{array}{l}\text { einfacher/mittlerer Angest./Beamter } \\
\text { höherer Angestellter/Beamter }\end{array}$ \\
\hline os & selbetändig, freiberuflich tätig \\
\hline$\infty$ & Lehrling/Umachüler \\
\hline 07 & Schüler/Student \\
\hline oa & $\begin{array}{l}\text { Hausfrau/Hausmann } \\
\text { sonstiges }\end{array}$ \\
\hline 90 & unbekannt \\
\hline
\end{tabular}

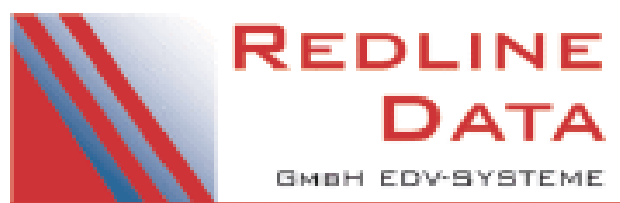

210 Vorgespräch in der Klinilk
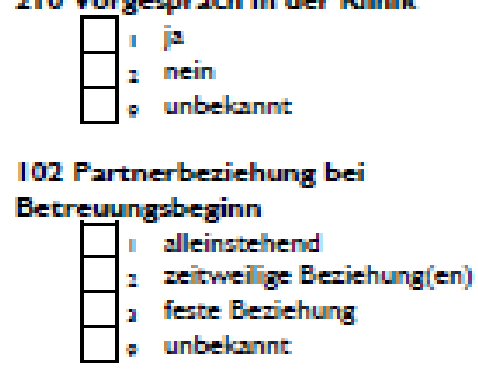

104 zeitlich überwiegende Wohnsituation in den letzten 6 Monaten

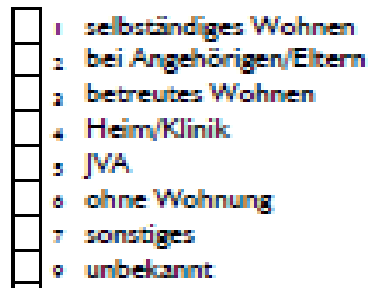

106 Abgeschlossene Berufsausbildung

$\square$ in Berufausbildung/Umochulung
2 Berufsausbildung abgeschlossen
2 Berufsausbildung abgebrochen
4 keine Ausbildung
: sonetiges
, unbelannt

108 Erwerbstätigkeit in den letzten

6 Monaten vor Betreuungsbeginn

\begin{tabular}{|l} 
\\
\hline \\
\hline \\
\hline \\
\hline \\
\hline \\
\hline
\end{tabular}

Auszubildende(r)

on Angestellte(r), Beamte(r)

on Arbeiter, Facharbeiter

Selbetändige(r), Freiberufler

sithelfende(r) Familienangehörige(r)

as Arbeiteloce( $(r)$

Schüler/Student

a Hausfrau/Hausmann

$\infty$ Rentner

a berufliche Rehabilitation

I sonstige Erwerbsloce

12 sonstiges

unbekannt 


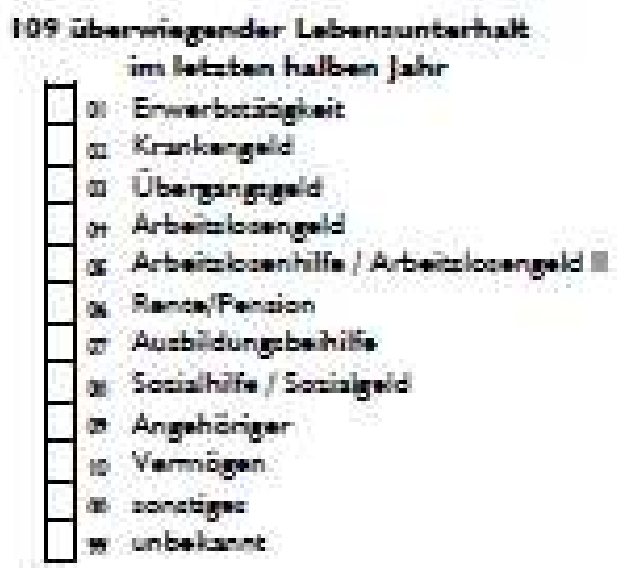

112 Rentematuation be Betrouangteginn

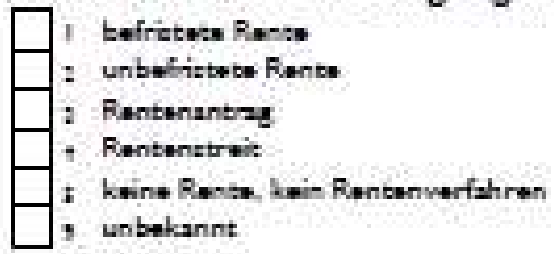

114 Yermittung

\begin{tabular}{|c|c|}
\hline ค & - - Farmatung \\
\hline 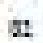 & Famnieffrounde \\
\hline ت & Arbetgetbr, Betritas Shule \\
\hline at & Windergl Ant Prychet-erspeut \\
\hline a; & Kra-Len-hou \\
\hline at & tabe-än Sud-tinnshbung \\
\hline का & Suchthrang: \\
\hline$x$ & siden Berah-ypdinats \\
\hline at & 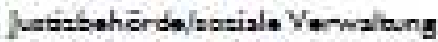 \\
\hline 10 & Alstinero-154lothilngrupet \\
\hline 1 & Kete--Lwithngtryjtr \\
\hline 12 & sontegt \\
\hline$n$ & I-bulant \\
\hline
\end{tabular}

116 Buchtproblume bei Euzugptraenn roithenden bei:

\begin{tabular}{|c|c|}
\hline \% & Leint \\
\hline I & Eheparthe-foberigeshresin] \\
\hline I & Vater \\
\hline 1 & Murther \\
\hline+ & Grabtin \\
\hline i & Geahmiater \\
\hline 4 & Kunder \\
\hline I & andere nahe Verwandte \\
\hline & unbelann: \\
\hline
\end{tabular}

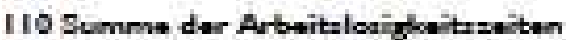
innertulb der lateten dra jahre vor Betrwungeteginn

Manate

III Gumme der uruntertbrecheren Arbaitalonighit unmittelby vor E-traungeb-ginn T Monste

113 jemala nuror

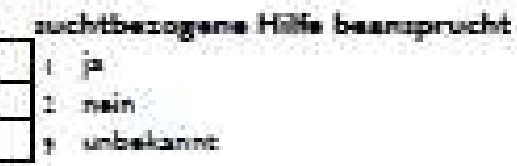

II 5 gattiche Grindagu

\begin{tabular}{|c|c|}
\hline & fre \\
\hline & trat \\
\hline & $\operatorname{ten}$ \\
\hline & $i$ ander strife-rlehe Grund ay \\
\hline & it Jihehtiche Grundige \\
\hline & Prych-Kgilundesh-tetringungrge: \\
\hline & unbelann: \\
\hline
\end{tabular}

Yarthgung

II7 Datum der VeHegurg

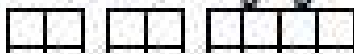

IIS Vtripgeng in Gropt

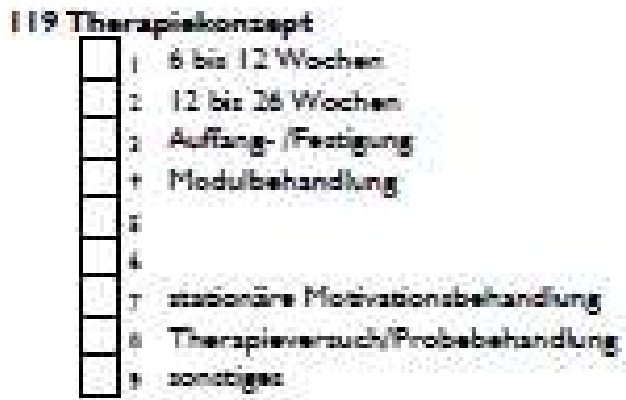




\section{Stammgruppe}

120 Familiensitustien bei Eqtrecungrende

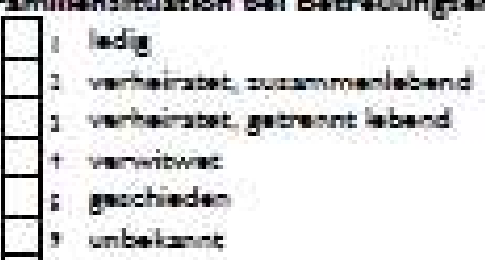

122 ubareiegandt Wohnituation bei Betrouungende

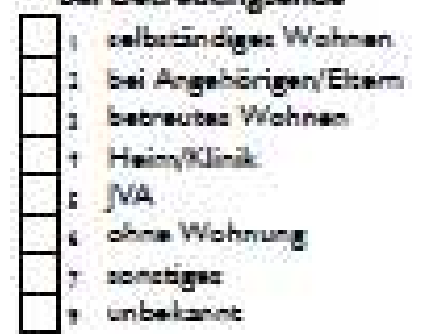

124 Rentenuituation beil Betreuungende

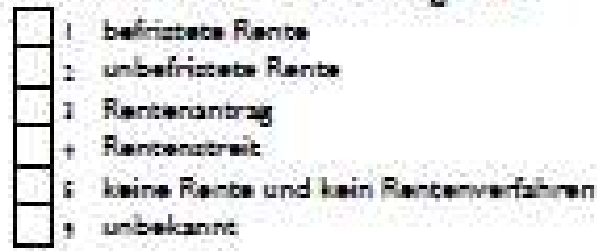

$12 \mid$ Patherterishung bei Butruungends

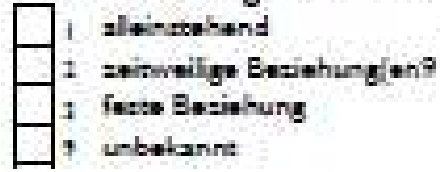

123 beruflide Integation nad Entlusung

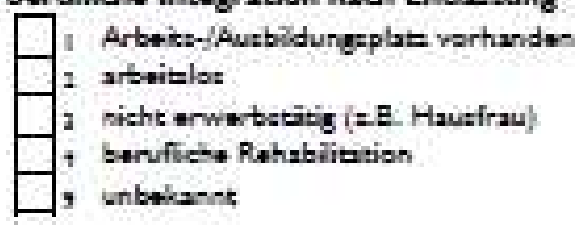

125 gobale dinguatische giturtion Hehiridinem-ung maghe

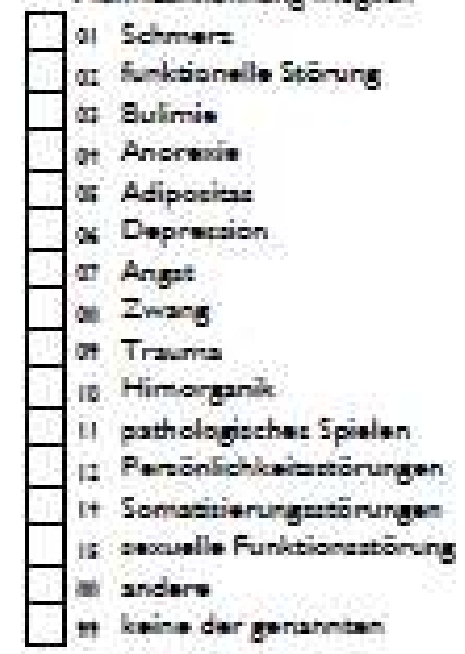

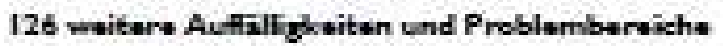
Mehitohne-nung reglith

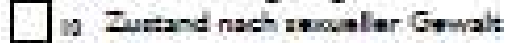

10 eprathikh Yertindigurgroblem.

n: Enschrinkungen dureh keperbelinderingen

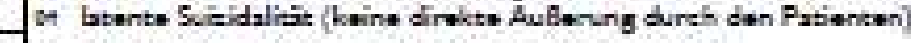

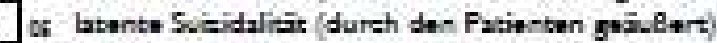

it aviste Handlungen in der Vorguetiahse

at Hohalmizbrzuch

10 Dregtrmiabrawh

s) Mhesmiztbrath

1) Medikammbunmiabrosh

Deir in de-Vorgtechishte

I2 Kamplinfal in der Vorpehibha

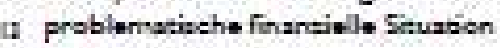

7) ktine der genannten 
127 Abtingigkitudawer

Quhn

129 schidivher Kensum/Abhinggktit

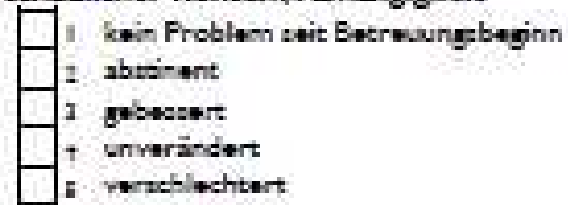

125 Sudtmithenam

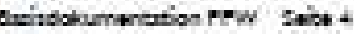

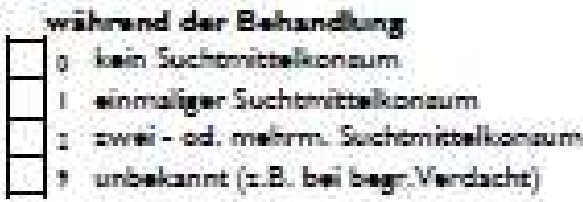

130 Art der Butrwurg

Mehtohne-nurg nisglis

$\square$ i ambulate Burang B--adung

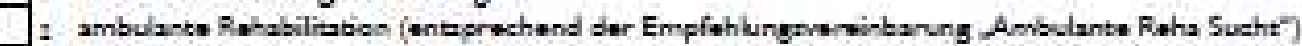

1 E-githung Entu-g

Subzibutionsbra-dung

ratanaw Entw Shnur gahondur g

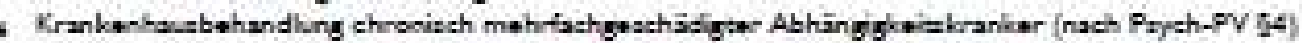

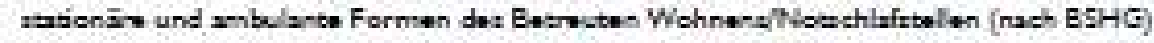

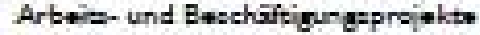

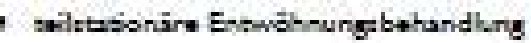

13 Kondahtrah

Karatte

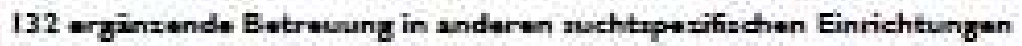

Mehtathmemung nesiah

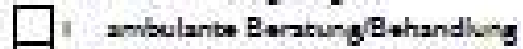

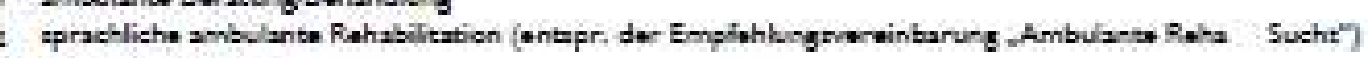

Ensphing E-eug

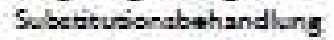

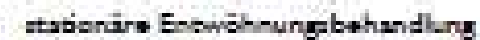

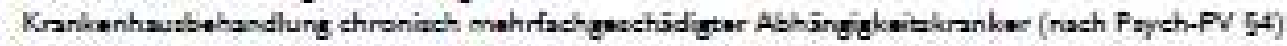

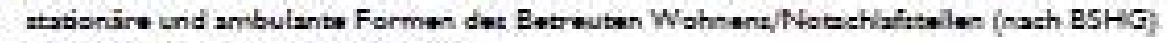

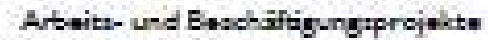

7) hine der genaninten

133 Yarehlig for rachfolgende MhBnuhnen

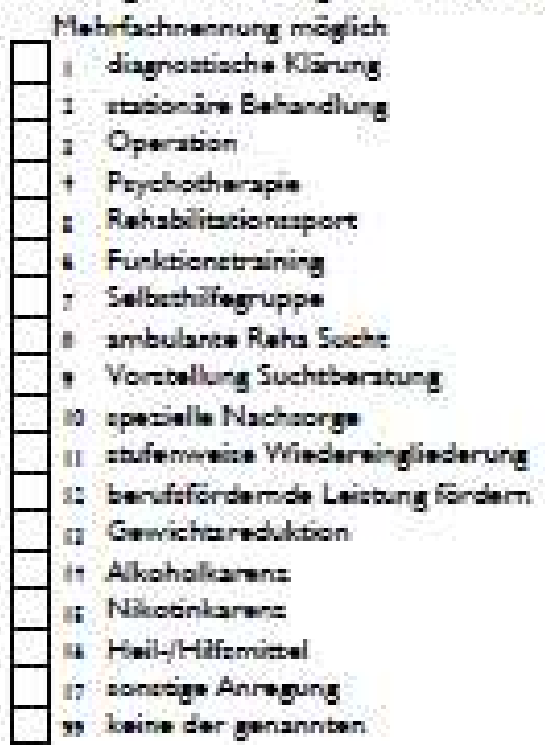


134 Weitartahandung aingalstet

Mehrohne-nury meglich

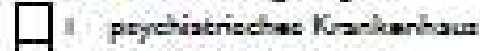

wenaju: Kra-kenha:

Sodholini:

andere Fahsilin

Adapsenswinfortung

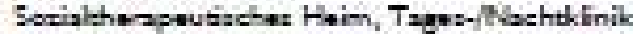

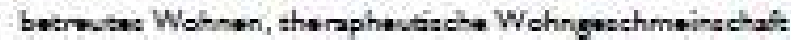

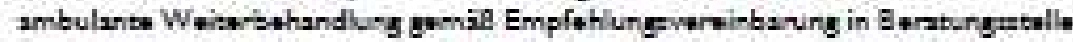

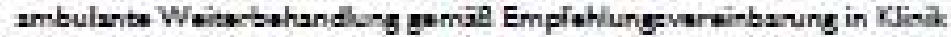

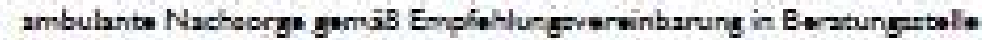

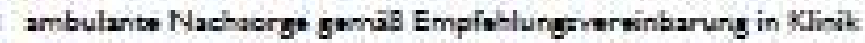

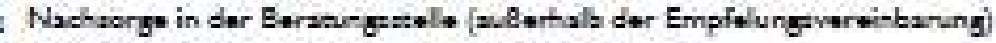

Fuchothespit bei rikderghasent Therapturen

benfiferdemde $\mathrm{H}=\mathrm{B}-\mathrm{a}-\mathrm{m}+\mathrm{n}$

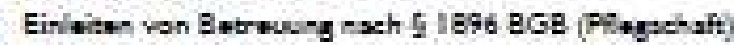

in lene der genannten

135 G*amtwinathitung der Yarindmrung

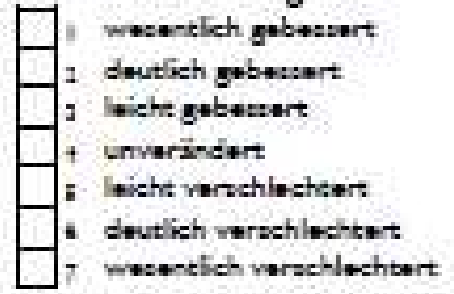

Auzt

140 Artatanhighat unmittalber ver Betsungbeginn

$\square$

attituring

arteitaunfaig

Hastiaultauman

Bt twing nide efendertis

(=E. Altortunter)

142 Dhus der ununtertmohanen

Artsitanfighighet unmittelbar

vor Betruung begin

पD Woden

143 Leitungerihigheit

im letten Berul ror Butruungeb-ginn

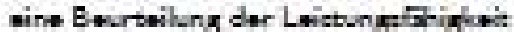

$\theta^{\prime}$

leg bei Extrwungibejoin nishever

s Stunde und me-

2 bia unte- S Sunden

Intar 3 Shinden

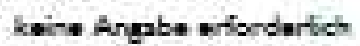

\section{Aufnahmetatua}

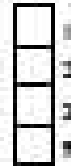

$$
\begin{aligned}
& \text { rar A-fhahme e-piftet } \\
& \text {-iaht morts: } \\
& \text { E-githung -isht erforderich }
\end{aligned}
$$

I3s Prognses

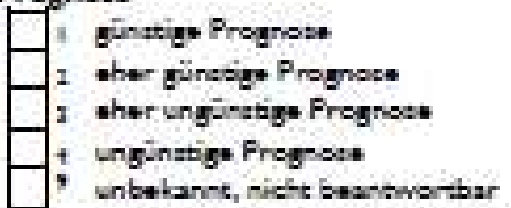

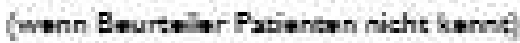

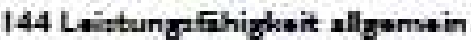
nor Butrauurgbagin

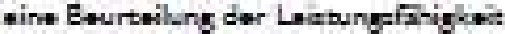

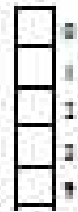

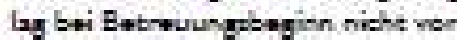
C Sunde- und me-r 3 bis unrer t Stunden unter $35 u-d e n$ ke-t H-gbe +ronderteh
}

146 Buchtmittal bei Aufnahm

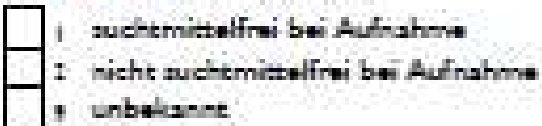


147 Etherige

tationim Entgfturgabehandlungen

17

149 Eisherige Subatitutionsthendlungen

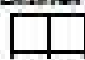

|5| bisharige

arrbulente Entwahnungbehandhingen

153 bizherigt

tationin Entrohnungtbahandhngtr T

155 bisherige taibtationare

Entmohnungthehand knger

0
148 Abschluse der heter

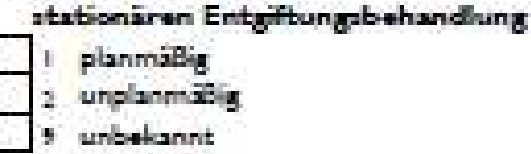

150 Abschluse der

$$
\text { Inten Bubatitutensthandhing }
$$

i planmiey

2. unglanmagy

, urbetannt

152 Abschluen der leteter

umbulantan Entwahnung behandlung

Qi pinmaly

- Inplanmilig

4 unbetert

154 Abschluen der leteter

atntianiren Entwahnung bahandlung

Qi planmity

2 urplinnsy

y urbthant

156 Abschluse der leteten telutationiren

Entwahmunglbehandlungen

Q planmity

2 urpla-mag

9 unbelannt

157 Atbeitafihigket bei Betreuungende

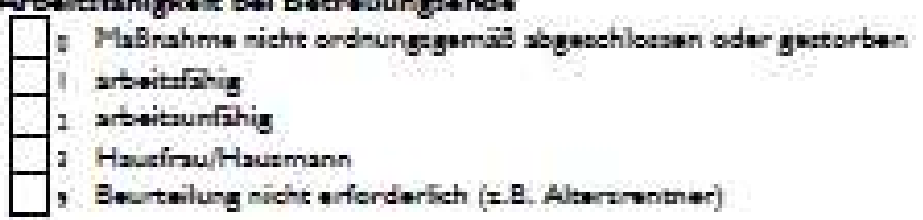

158 interkurnete Arteiteunfihighit bei Betreusugend.

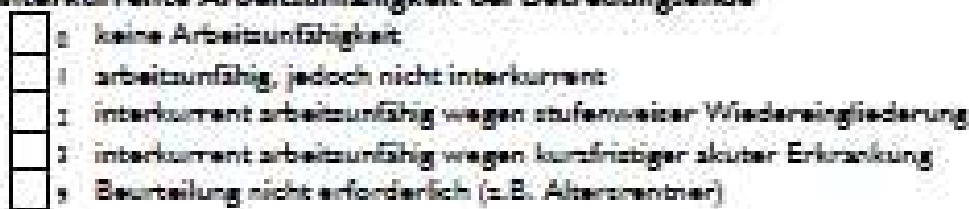

159 Lwitungahigkeit fur latrten Eeruf bei Betroungend.

$\theta$

A Sturden urd mah

2 tia unter 6 Stunden

unitr 3 Sbindr.

thint Angbe- wardetiah

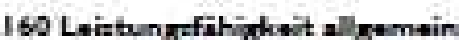
bei Eatrumungendy

I 6 Stum-tn u-d $m+h r$

23 bie unter 6 shnden

i unter 3 stunde-

, hen Angate wriardeteh

16. Enthasungform

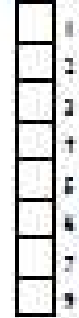

Nyily

rerequg auf irshe-t Wers-lasu-g

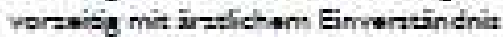

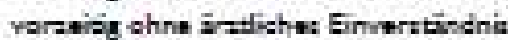

=ipiplingiseh

rarty

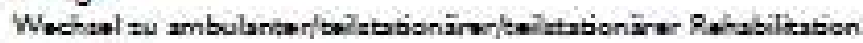

ga:mbr- 


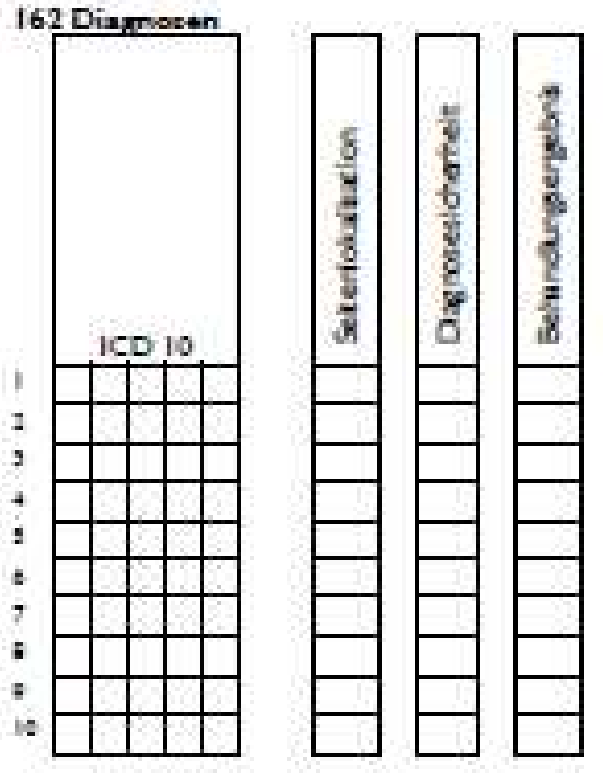

Risikofaktoren

I Alucher?<smiles>C1CC2CCC12</smiles>

1. Minin

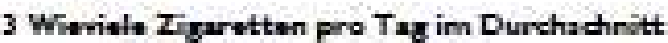

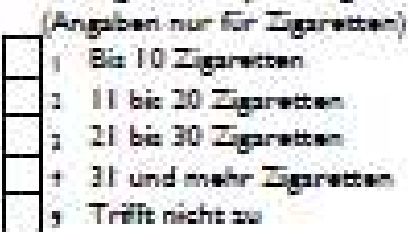

4 Quatwht win Anderungmunseh Fr dan dartitigan Konaum

Q. Kr-Andeungmunteh

1) $b_{1}$ Revalution

1. j, gans a-ten

1. Trift nitht $x$
Stimblatuter

E Exilutity

L Linkews.

R Rechereate

St=hethen

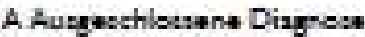

G Geikhette Diagneat

VWerdachadaynest

Z Zunte-d nseh

Enptria

Oerft nehts

gutent

2 unstinde-

3 warelehtert
2 Art dus Tabalbonauma

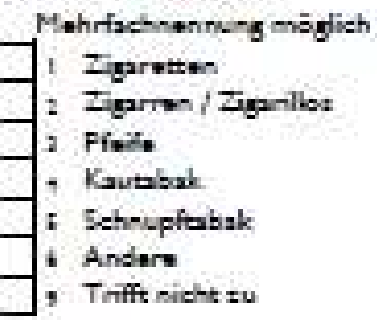

5 Hat aikh dar Tabukboum weindert whitrend der Behendung

o Keine Andering

it je, ratuits

It pare sufghiort

a j, itat

Trift meht zu 


\section{Anhang 4}

\section{Katamneseanschreiben}

Diakonie-Krankenhaus

Harz GmbH - Brockenstr. 1 - 38875 Elbingerode

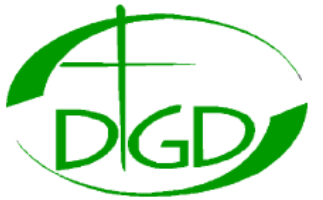

DIAKONIE-

KRANKENHAUS

ELBINGERODE

Deutscher Gemeinschafts-Diakonieverband

$\mathrm{KTQ}+$ proCum Cert

\begin{tabular}{|c|c|c|}
\hline $\begin{array}{l}\text { Thre Zeichen } \\
\text { Thre Nachricht vom }\end{array}$ & $\begin{array}{l}\text { Unsere Zeichen } \\
\text { Unsere Nachricht vom }\end{array}$ & $\begin{array}{l}\text { Telefon } \\
(039454)\end{array}$ \\
\hline
\end{tabular}

Sehr geehrter Herr Mustermann

vor einiger Zeit waren Sie in unserer Klinik in Behandlung und wir hoffen, dass Sie auch aus jetziger Sicht Ihre Entscheidung für die Therapie als richtig und gut einschätzen. Als Fachklinik für die Behandlung von Suchterkrankungen sind wir angehalten und auch interessiert zu erfahren, ob und wie weit die Behandlung für Sie förderlich war. Wir befragen deshalb unsere früheren Patienten im Abstand von ca. einem und vier Jahren nach Beendigung der Behandlung. Unser Anliegen ist, so Hinweise zu bekommen, in welchen Bereichen unsere Behandlung zu verbessern ist. Von Ihren Erfahrungen profitieren dann die zukünftigen Patienten. Deshalb möchten wir Sie bitten, diese ca. 15 Minuten zu investieren und uns zu helfen, damit wir besser helfen können, herzlichen Dank.

Es wurde versucht, den Fragebogen verständlich zu gestalten. Bei jeder Frage steht ein Hinweis, wie sie auszufüllen ist. Bitte kreuzen Sie das oder die zutreffenden Kästchen an. An einigen Stellen bitten wir Sie, auch zusätzliche Anmerkungen zu machen. Kommentare zum Fragebogen oder andere Dinge, die Sie uns mitteilen wollen, können Sie auch in den Rückumschlag legen. Bitte füllen Sie den Fragebogen vollständig aus.

Wir versichern Ihnen, dass Ihre Angaben vertraulich behandelt werden entsprechend den gesetzlichen Datenschutzbestimmungen

Bitte legen Sie den ausgefüllten Fragebogen in den beiliegenden Umschlag und schicken Sie diesen an unsere Klinik zurück. Das Porto übernehmen selbstverständlich wir.

Wenn sich noch Fragen ergeben sollten, antworten wir gerne.

Noch einmal danke für lhre Mitarbeit, wir grüßen herzlich und wünschen Ihnen einen guten Weg.

Im Auftrag der Mitarbeiter

$\mathrm{lhr}$

Dr. med. Richter

FA für Neurologie

und Psychiatrie

(Ltd. Chefarzt)
Diakonie-Krankenhaus

$\mathrm{Harz} \mathrm{GmbH}$

Brockenstr. 1

38875 Elbingerode

Geschäftsführer

Martin Montowski

Anita Rost

Vorsitzender des

Aufsichtsrates:

Andreas Heinemann

Handelsregister:

Amtsgericht

Magdeburg

HRB 8777 
Nachbefragungsbogen (AHG - Wissenschaftsrat, 2002 - Programmversion

1.0; schriftliche Form übernommen aus dem Patfak für Windows Handbuch, Stand 31.12.2004)

\section{Diakonie-Krankenhaus Harz GmbH}

- Medizinische Rehabilitation Sucht -

Brockenstr. 1, 38875 Elbingerode

Datum :

1. Wie ist Ihr derzeitiger Familienstand?

Code - Nummer

(Eine Antwort ist möglich)

ledig

$\square \quad 1$

verwitwet

$\square$

verheiratet; zusammenlebend

2

geschieden

$\square$ 5

verheiratet; getrenntlebend

$\square$

2. Wie sah im letzten halben Jahr Ihre Tätigkeit aus?

(Eine Antwort ist möglich)

Auszubildende / $\mathbf{r}$

Arbeitsbeschaffungs-

Maßnahme (ABM)

Angestellte / $\mathrm{r}$

Beamter / in

Arbeiter / in

Facharbeiter / in

Selbständige /r, Freiberufler/in
Mithelfende/r Familien- $\square 8$

Angehörige/r

Erwerbslose/r

Schüler/in, Student/in $\square 10$

Hausfrau/-mann $\quad \square 11$

Rentner/in, Pensionär/in $\square 12$

Sonstiges: $\quad \square 13$

3. Wenn Sie im letzten halben Jahr arbeitslos waren, wie viele Monate sind/waren Sie es?

4. Haben Sie im letzten halben Jahr folgende Ausbildungen oder Maßnahmen begonnen/beendet?

(Pro Zeile ist eine Antwort möglich) bitte ankreuzen

\begin{tabular}{|l|l|l|l|l|}
\hline Ausbildung & keine & noch in... & abgebrochen & abgeschlossen \\
\hline Schulausbildung & & & & \\
\hline Berufsausbildung (Lehre) & & & & \\
\hline Berufsbildende Maßnahme * & & & & \\
\hline
\end{tabular}

*berufsbildende Maßnahmen sind z.B. EDV- Kurse und Lehrgänge für Kurzschrift oder Maschinenschreiben usw. 
5. Wovon haben Sie im letzten halben Jahr Ihren Lebensunterhalt überwiegend bestritten?

(Eine Antwort ist möglich)

Erwerbstätigkeit

$\square \quad 1$

Ausbildungsbeihilfe

Krankengeld

2

Sozialhilfe

7

Übergangsgeld

3

Angehörige/r

8

Arbeitslosengeld

Vermögen

9

Arbeitslosenhilfe

Sonstiges

10

Rente/ Pension

6. Wie zufrieden waren Sie im letzten halben Jahr mit Ihrer beruflichen/ schulischen Situation?

(bitte geben Sie auch Auskunft, wenn Sie im letzten halben Jahr nicht erwerbstätig waren z.B. Hausfrau/-mann, Rentner/in, Schüler/in, arbeitslos)

\begin{tabular}{|c|c|c|c|c|}
\hline$\square 1$ & $\square^{2}$ & $\square^{3}$ & $\square 4$ & $\square 5$ \\
\hline $\begin{array}{l}\text { sentlich } \\
\text { bessert }\end{array}$ & verbessert & gleichbleibend & verschlechtert & $\begin{array}{l}\text { wesentlich } \\
\text { verschlechtert }\end{array}$ \\
\hline
\end{tabular}

7. Wie bewerten Sie insgesamt seit Beendigung der Behandlung in unserer Einrichtung Ihre berufliche/schulische Situation?

\begin{tabular}{|c|c|c|c|c|}
\hline$\square 1$ & $\square^{2}$ & $\square^{3}$ & $\square 4$ & $\square 5$ \\
\hline $\begin{array}{l}\text { wesentlich } \\
\text { verbessert }\end{array}$ & verbessert & gleichbleibend & verschlechtert & $\begin{array}{l}\text { wesentlich } \\
\text { verschlechtert }\end{array}$ \\
\hline
\end{tabular}

8. Mit wem haben Sie im letzten halben Jahr zusammengelebt (Mehrere Antworten sind Möglich)

Partner/in

Kind/ern

Eltern

Anderen Angehörigen
Freunden/Bekannten

Sonstigen

Trifft nicht zu, lebe allein verschlechtert 
9. Wo haben Sie im letzten halben Jahr gewohnt?

(Eine Antwort ist möglich)

In eigener/gemieteter

Wohnung/Haus

Heim/Anstalt/Klinik $\quad \square_{4}$

In Straf-/Untersuchungshaft $\square 5$

In anderer Wohnung

Betreutes Wohnen

Wohnungslos

6

Sonstiges ....

10. Wie zufrieden waren Sie im letzten halben Jahr mit Ihrer Wohnsituation?

$\begin{array}{ccccc}\square_{1} & \square_{2} & \square{ }_{3} & \square{ }_{4} & \square{ }_{5} \\ \text { wesentlich } & \text { verbessert } & \text { gleichbleibend } & \text { verschlechtert } & \text { wesentlich } \\ \text { verbessert } & & & \text { verschlechtert }\end{array}$

\section{Wie zufrieden waren Sie im letzten halben Jahr mit Ihrer körperlichen} Verfassung?

$\begin{array}{ccccc}\square_{1} & \square_{2} & \square_{3} & \square{ }_{4} & \square{ }_{5} \\ \text { wesentlich } & \text { verbessert } & \text { gleichbleibend } & \text { verschlechtert } & \text { wesentlich } \\ \text { verbessert } & & & \text { verschlechtert }\end{array}$

12. Wie zufrieden waren Sie im letzten halben Jahr mit Ihrer seelischen Verfassung

\begin{tabular}{|c|c|c|c|c|}
\hline $\begin{array}{l}\square 1 \\
\text { wesentlich } \\
\text { verbessert }\end{array}$ & $\begin{array}{c}\square 2 \\
\text { verbessert }\end{array}$ & $\begin{array}{l}\square 3 \\
\text { gleichbleibend }\end{array}$ & $\begin{array}{c}\square 4 \\
\text { verschlechtert }\end{array}$ & $\begin{array}{l}\quad \square 5 \\
\text { wesentlich } \\
\text { verschlechtert }\end{array}$ \\
\hline
\end{tabular}

13.In welcher Partnerbeziehung haben Sie im letzten halben Jahr gelebt? (Eine Antwort ist möglich)
Alleinstehend
1
Zeitweilige Beziehung (en)
2
Feste Beziehung

14. Wie zufrieden waren Sie im letzten halben Jahr mit Ihrer Ehe/PartnerSituation?

\begin{tabular}{|c|c|c|c|c|}
\hline $\begin{array}{l}\square 1 \\
\text { wesentlich } \\
\text { verbessert }\end{array}$ & $\begin{array}{c}\square 2 \\
\text { verbessert }\end{array}$ & $\begin{array}{c}\square 3 \\
\text { gleichbleibend }\end{array}$ & $\begin{array}{c}\square 4 \\
\text { verschlechtert }\end{array}$ & $\begin{array}{l}\quad \square 5 \\
\text { wesentlich } \\
\text { verschlechtert }\end{array}$ \\
\hline
\end{tabular}


15. Wie zufrieden waren Sie im letzten halben Jahr mit Ihren Kontakten zu Freunden und Bekannten?

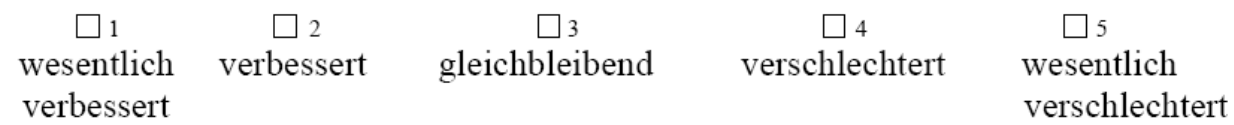

\section{Gab es für Sie im letzten halben Jahr Erlebnisse, die für Sie positiv oder belastend waren? \\ (Mehrere Antworten sind möglich)}

\begin{tabular}{|l|l|l|}
\cline { 2 - 3 } \multicolumn{1}{c|}{} & \multicolumn{2}{c|}{ Zutreffendes bitte ankreuzen } \\
\hline Neuer Arbeitsplatz & Positiv & negativ \\
\hline Verlust der Arbeit & & \\
\hline Prüfung & & \\
\hline Neue Beziehung & & \\
\hline Heirat & & \\
\hline Geburt eines Kindes & & \\
\hline Genesung von einer Krankheit & & \\
\hline Krankheit/Unfall/Behinderung & & \\
\hline HIV- Testergebnis erfahren & & \\
\hline Kein Rückfall & & \\
\hline Rückfall & & \\
\hline Stationäre psychiatrische Behandlung & & \\
\hline Entlassung aus stationärer psychiatrischer Behandlung & & \\
\hline Gerichtsverhandlung & & \\
\hline Verhaftung/Gefängnis & & \\
\hline Entlassung aus Haft & & \\
\hline Tod eines nahestehenden Menschen & & \\
\hline Wechsel der Wohnung/neue Wohnung & & \\
\hline Verlust der Wohnung & & \\
\hline Ende einer persönlichen Beziehung & & \\
\hline Suizidversuch & & \\
\hline Sonstiges Ereignis & & \\
\hline
\end{tabular}


17. Haben Sie seit Beendigung der Behandlung in unserer Einrichtung, bzw. im letzten halben Jahr Suchtmittel zu sich genommen?

Wir bitten Sie, in dieser Frage Auskunft über Ihren Alkohol-, Medikamenten- und Drogenkonsum zu geben.

Gehen Sie bitte Zeile für Zeile vor und beantworten Sie die Fragen für jedes Suchtmittel getrennt. Beantworten Sie erst die Fragen, ob Sie Suchtmittel dieser Art genommen haben. Wenn Sie mit ,ja“ antworten, beantworten Sie bitte auch alle anderen Fragen der Zeile und gehen dann zur nächsten Zeile über. Wenn Sie mit „nein“ geantwortet haben, können Sie gleich zur nächsten Zeile gehen. Bei Medikamenten- und/oder Drogenkonsum bitten wir Sie in Frage 18 um genauere Angaben zu den konsumierten Substanzen.

(Mehrere Antworten sind möglich)

\begin{tabular}{|c|c|c|c|c|}
\hline $\begin{array}{l}\text { Art des } \\
\text { Suchtmittels }\end{array}$ & $\begin{array}{l}\text { Haben Sie } \\
\text { Suchtmittel } \\
\text { dieser Art } \\
\text { genommen? }\end{array}$ & $\begin{array}{l}\text { Wie viele } \\
\text { Wochen sind Sie } \\
\text { nach } \\
\text { Behandlungsende } \\
\text { durchgehend } \\
\text { abstinent } \\
\text { geblieben? }\end{array}$ & $\begin{array}{l}\text { Wie viele } \\
\text { Wochen sind Sie } \\
\text { im letzten halben } \\
\underline{\text { Jahr }} \\
\text { durchgehend } \\
\text { abstinent } \\
\text { geblieben? }\end{array}$ & $\begin{array}{l}\text { Seit wie vielen } \\
\text { Wochen sind } \\
\text { Sie jetzt wieder } \\
\text { durchgehend } \\
\text { abstinent? }\end{array}$ \\
\hline Alkohol & $\begin{array}{cc}\text { ja } & \text { nein } \\
\square & \square\end{array}$ & & & \\
\hline $\begin{array}{l}\text { suchterzeugende } \\
\text { Medikamente } \\
\text { Gegen bzw. ohne } \\
\text { Anordnung durch } \\
\text { den Arzt }\end{array}$ & $\begin{array}{rr}\text { ja } & \text { nein } \\
\square & \square\end{array}$ & & & \\
\hline Drogen & $\begin{array}{rr}\text { ja } & \text { nein } \\
\square & \square\end{array}$ & & & \\
\hline Tabak & $\begin{array}{rr}\text { ja } & \text { nein } \\
\square & \square\end{array}$ & & & \\
\hline
\end{tabular}

\section{Wenn Sie Medikamente (in nicht bestimmungsmäßigem Gebrauch) und/oder Drogen im letzten halben Jahr zu sich genommen haben, welche Suchtmittel waren dies? \\ (Mehrere Antworten sind möglich)}

Heroin.

Methadon

Codein-Präperate

Sonstige opiathaltige Mittel

Haschisch/Marihuana.

Schlafmittel.

Beruhigungsmittel

Schmerzmittel.
Kokain

Crack.

Andere kokainhaltige Mittel.

Aufputschmittel.

LSD.

Mescalin.

Sonstige Designer Drugs.

andere psychoaktive Substanzen,

wenn ja, welche. 
19. Falls Sie im letzten halben Jahr Suchtmittel (ausgenommen Nikotin) konsumiert haben, welche Situationen/Bedingungen/Einstellungen haben dazu beigetragen?

(Mehrere Antworten sind Möglich)

Situation im Arbeitsleben.

Situation in der Partner-

schaft / Familie

Gesundheitliche Probleme.

Gesteigertes Verlangen nach

Suchtmitteln

Probleme mit Polizei und Justiz.

Streit, Ärger, Frust.

Langeweile.

Isolation, Einsamkeit
Direkte Suchtmittelangebote

Konsum, um positive Wirkungen/ Gefühle zu erzielen.

Konsum, um Willensstärke zu testen

Konsum wegen freudiger Ereignisse, gehobener Stimmung und Glücksgefühlen

Konsum ist für mich kein Problem

Sonstiges

20. Wie beschreiben Sie selbst im letzten halben Jahr Ihren Umgang mit Suchtmitteln (ausgenommen Nikotin)?

(Eine Antwort ist möglich)

Kein Suchtmittelkonsum mehr

Täglicher/ fast täglicher Konsum

Nur gelegentlicher Konsum

Täglicher mehrmaliger Konsum

\section{Sind Sie seit Beendigung der Behandlung in unserer Einrichtung im} Zusammenhang mit Suchtmitteln verurteilt worden?

(Mehrere Antworten sind möglich)

Keine Verurteilung

Verkehrsdelikte

Führerscheinentzug

Sachbeschädigung
Delikte gegen Personen

Beschaffungsdelikte

BtM-Delikte 
22. Haben Sie im letzten halben Jahr Eßstörungen gehabt und/oder an Automaten gespielt, bzw. an Glücksspielen teilgenommen?

(Mehrere Antworten sind möglich)

\begin{tabular}{|l|l|l|l|}
\hline & $\begin{array}{l}\text { Haben Sie nichtstoff- } \\
\text { gebundene Formen des } \\
\text { Konsums/Sucht- } \\
\text { verhaltens gehabt? }\end{array}$ & $\begin{array}{l}\text { Wie viele Wochen sind } \\
\text { Sie im letzten halben } \\
\text { Jahr durchgehend } \\
\text {,abstinent" geblieben? }\end{array}$ & $\begin{array}{l}\text { Seit wie vielen } \\
\text { Wochen sind Sie jetzt } \\
\text { wieder durchgehend } \\
\text {,abstinent“? }\end{array}$ \\
\hline Eßstörungen & $\square$ nein $\square$ ja & & \\
\hline $\begin{array}{l}\text { Pathologisches } \\
\text { Spielen }\end{array}$ & $\square$ nein $\square$ ja & & \\
\hline
\end{tabular}

\section{Geben Sie bitte an, wie häufig Sie im letzten halben Jahr eine oder mehrere der genannten Institutionen aufgesucht haben. \\ (Mehrere Antworten sind möglich)}

\begin{tabular}{|c|c|c|c|c|c|}
\hline & $\begin{array}{l}\mathbf{1 x} \text { pro } \\
\text { Woche } \\
\text { oder öfter }\end{array}$ & \begin{tabular}{|l|}
$\mathbf{2 - 3 x}$ \\
pro Monat
\end{tabular} & $\begin{array}{l}1 \mathbf{x} \text { pro } \\
\text { Monat }\end{array}$ & \begin{tabular}{|l} 
seltener als \\
$\mathbf{1 x}$ \\
pro Monat
\end{tabular} & $\begin{array}{l}\text { nie } \\
\text { aufgesucht }\end{array}$ \\
\hline Selbsthilfegruppe, welche : & $\square$ & $\square$ & $\square$ & $\square$ & $\square$ \\
\hline Beratungsstelle/ Ambulanz & $\square$ & $\square$ & $\square$ & $\square$ & $\square$ \\
\hline $\begin{array}{l}\text { Niedergelassene/r } \\
\text { Psychotherapeut/in }\end{array}$ & $\square$ & $\square$ & $\square$ & $\square$ & $\square$ \\
\hline Sonstiges, bitte angeben : & & & & & \\
\hline Stationäre Entgiftung & \multicolumn{3}{|c|}{ Anzahl : } & \multirow{2}{*}{\multicolumn{2}{|c|}{ bis _(in Monat/Jahr) }} \\
\hline & \multicolumn{3}{|c|}{ von... (Angabe in Monat/Jahr) } & & \\
\hline \multicolumn{6}{|l|}{ Stationäre Entwöhnung } \\
\hline \multicolumn{6}{|l|}{ Adaption } \\
\hline \multicolumn{6}{|l|}{ Stationäre Nachsorge } \\
\hline \multicolumn{6}{|l|}{ Tages-/Nachtklinik } \\
\hline \multicolumn{6}{|l|}{ Jugendhilfewohngruppe } \\
\hline \multicolumn{6}{|l|}{ Betreutes Wohnen } \\
\hline Sonstiges, bitte angeben : & & & & & \\
\hline
\end{tabular}

\section{Welches Ziel im Umgang mit dem/den Suchtmittel/n, wegen dem/denen Sie} behandelt wurden, haben Sie für sich selbst festgelegt?

(Eine Antwort ist möglich)

Kein Suchtmittelkonsum

Konsum nur bei besonderen

Gelegenheiten (Feste, offizielle Anlässe) $\square_{2}$
Kontrollierter Konsum $\quad \square_{3}$

Konsum ohne Einschränkung $\quad \square_{4}$ 
25. Wie haben Sie es geschafft, Ihre selbstgesetzten Ziele im Umgang mit dem/den Suchtmittel/n, wegen dem/denen Sie behandelt wurden, zu erreichen?

(Mehrere Antworten sind möglich)

$\begin{array}{lll}\begin{array}{l}\text { Es ist mir nicht gelungen, } \\ \text { meine Ziele zu erreichen }\end{array} & \square & \begin{array}{l}\text { Durch Unterstiutzung von Betrieb } \\ \text { und/oder Kollegen/innen }\end{array} \\ \begin{array}{l}\text { Der Grund ist mir selbst } \\ \text { nicht erklärlich }\end{array} & \square & \begin{array}{l}\text { Durch Unterstïtzung von Arzt } \\ \text { und/oder Beratungsstelle }\end{array} \\ \text { Durch eigenen Willen } & \square & \begin{array}{l}\text { Durch Unterstiutzung einer } \\ \text { Selbsthilfegruppe }\end{array} \\ \begin{array}{l}\text { Durch Unterstiutzung von } \\ \text { Partner/in und/oder Familie }\end{array} & \square & \text { Durch Anderes und zwar : }\end{array}$

26. Wie würde ein Ihnen nahestehender Mensch Ihre jetzige Lebenssituation beurteilen?

\begin{tabular}{|c|c|c|c|}
\hline $\begin{array}{l}\quad \square^{1} \\
\text { wesentlich } \\
\text { verbessert }\end{array}$ & $\begin{array}{c}\square^{2} \\
\text { verbessert }\end{array}$ & gleichbleibend & $\begin{array}{c}\square^{4} \\
\text { verschlechtert }\end{array}$ \\
\hline
\end{tabular}

\section{Vielen Dank für Ihre Mitarbeit !}

$\triangle$ Raum für Bemerkungen, Mitteilungen : 


\section{Anhang 5}

Tabellen

Im Folgenden finden sich alle Tabellen der Rohdaten der linearen und der logistischen Regressionen aus der Untersuchung auf Prädiktoren für den Behandlungserfolg von Alkoholabhängigen des Jahrgangs 2004 des Diakoniekrankenhauses Harz GmbH. Sie beziehen sich auf die Tabelle 3 im Text und enthalten die dort aufgeführten Einzelwerte.

\section{Zugang und Entlassform}

Tabelle 4: Zugang und Entlassform - Zusammenfassung der Fallverarbeitung der logistischen Regression

\begin{tabular}{|c|c|c|c|}
\hline \multicolumn{4}{|c|}{ Zusammenfassung der Fallverarbeitung } \\
\hline & & $\mathrm{N}$ & Prozent \\
\hline \multirow{3}{*}{$\begin{array}{l}\text { Ausgewählte } \\
\text { Fälle }\end{array}$} & Einbezogen in Analyse & 268 & 61,8 \\
\hline & Fehlende Fälle & 166 & 38,2 \\
\hline & Gesamt & 434 & 100,0 \\
\hline \multicolumn{2}{|c|}{ Nicht ausgewählte Fälle } & 0 & $\mid 0$ \\
\hline \multicolumn{2}{|c|}{ Gesamt } & 434 & 100,0 \\
\hline
\end{tabular}

Tabelle 5: Zugang und Entlassform - Codierung der abhängigen Variablen der logistischen Regression

\section{Codierung abhängiger Variablen}

\begin{tabular}{|l|l|l|}
\hline Ursprünglicher Wert & Interner Wert \\
\hline Entlassform & $\begin{array}{l}\text { irregulär } \\
\text { entlassen }\end{array}$ & 0 \\
\cline { 2 - 3 } & $\begin{array}{l}\text { regulär } \\
\text { entlassen }\end{array}$ & 1 \\
\hline
\end{tabular}

Tabelle 6: Zugang und Entlassform - Modellzusammenfassung der logistischen Regression; "Schritt 1" deutet darauf hin, dass die logistische Regression durchgeführt wurde. Nagelkerkes R-Quadrat besagt hier, dass durch die im Modell zusammengefassten Variablen immerhin 53,4\% der gemeinsamen Varianz aufgeklärt werden.

\begin{tabular}{|l|l|}
\hline Modellzusammenfassung \\
\hline Schritt & $\begin{array}{l}\text { Nagelkerkes } \\
\text { R-Quadrat }\end{array}$ \\
\hline 1 &, 534 \\
\hline
\end{tabular}


Tabelle 7: Zugang und Entlassform - Klassifizierungstabelle der logistischen Regression; „a“: der Trennwert lautet 0,500; die Tabelle gibt die Übereinstimmung zwischen der aus dem Modell vorhergesagten Entlassform und der beobachteten Entlassform wieder

\begin{tabular}{|c|c|c|c|c|}
\hline \multicolumn{5}{|c|}{ Klassifizierungstabelle $^{a}$} \\
\hline \multirow{3}{*}{ Beobachtet } & & \multicolumn{3}{|c|}{ Vorhergesagt } \\
\hline & & \multicolumn{2}{|c|}{ Entlassform } & \multirow{2}{*}{$\begin{array}{l}\text { Prozentsatz } \\
\text { der } \\
\text { Richtigen }\end{array}$} \\
\hline & & $\begin{array}{l}\text { irregulär } \\
\text { entlassen }\end{array}$ & $\begin{array}{l}\text { regulär } \\
\text { entlassen }\end{array}$ & \\
\hline \multirow{2}{*}{ Entlassform } & $\begin{array}{l}\text { irregulär } \\
\text { entlassen }\end{array}$ & 15 & 14 & 51,7 \\
\hline & \begin{tabular}{|l|} 
regulär \\
entlassen
\end{tabular} & 1 & 238 & 99,6 \\
\hline \multicolumn{2}{|c|}{ Gesamtprozentsatz } & & & 94,4 \\
\hline
\end{tabular}


Tabelle 8: Zugang und Entlassform - Variablen in der Gleichung nach durchgeführter logistischer Regression

\section{Legende:}

- $\quad$ "a“ = „Schritt 1“ gibt an, dass die logistische Regression durchgeführt wurde

- "RegressionskoeffizientB“ = gibt die Richtung des Effekts auf die abhängige Variable bei Erhöhung des Werts der unabhängigen Variablen um „1“ an

- "Wald“ = statistischer Test für die multiple Regressionsanalyse, der aus dem Verhältnis "Regressionskoeffizient B zum Quadrat / Standardfehler zum Quadrat" das Signifikanzniveau (= "Sig.") errechnet

- $\quad$ "df" = Freiheitsgrade

- ${ } \operatorname{Exp}(B))^{“}=$ gibt die Änderung des Chancenverhältnisses an bei Erhöhung der unabhängigen Variablen, wobei Werte $>1$ eine Erhöhung des Effekts bedeuten, Werte $<1$ eine Verminderung

- Grau hinterlegt sind die Variablen der Hypothese 1, darauf folgen die Variablen der Hypothese 2

- Als Dummy-Variablen gingen in die Berechnung ein: „Auflage“ („Auflage“ erhielt den Wert „1“), "Geschlecht“ („weiblich“ = „1“), „Wiederaufnahme“ (,Ja“ = „1“), „Zuvor suchtbezogene Hilfe (erhalten)“ (,Ja“ = „1“)

- In Dummy-Variablen umgewandelte kategoriale Variablen sind: "Motivationslage“ (Referenzkategorie (=RK) „Eigenmotiviert“), „Dauer_Arbeitslosigkeit“ (RK „Arbeitslosigkeit < 1 Monat"), „Erwerbssituation_Anfang“ (RK „erwerbstätig“), „Schulabschluss“ (RK „Abitur/(Fach)Hochschulreife“), „Kostenträger“ (RK „Bundesversicherungsanstalt für Angestellte"), „Familienstand“ (RK „verheiratet, zusammenlebend“)

- Alle anderen Variablen sind metrischer Natur

- $\quad$ „IQ“ = Intelligenzquotient, abgeschätzt anhand des Wortschatztests

- "c.I.-Skala“ = Skala zur Erfassung cerebraler Insuffizienzen

- $\quad$ "PSSI“ = Persönlichkeits-Stil-und-Störungs-Inventar

- "LVA“ = Landesversicherungsanstalt, der frühere Name der regionalen Rentenversicherungen

\begin{tabular}{|c|c|c|c|c|c|c|c|c|c|}
\hline \multicolumn{10}{|c|}{ Variablen in der Gleichung } \\
\hline & & \multirow{2}{*}{$\begin{array}{l}\text { Regres- } \\
\text { sionsko- } \\
\text { effizientB }\end{array}$} & \multirow{2}{*}{$\begin{array}{l}\text { Standard } \\
\text { fehler }\end{array}$} & \multirow[t]{2}{*}{ Wald } & \multirow[t]{2}{*}{ df } & \multirow[t]{2}{*}{ Sig. } & \multirow[t]{2}{*}{$\operatorname{Exp}(B)$} & \multicolumn{2}{|c|}{$\begin{array}{l}95 \\
\text { Konfidenzintervall } \\
\text { für } \operatorname{EXP}(\mathrm{B})\end{array}$} \\
\hline & & & & & & & & \begin{tabular}{|l} 
Unterer \\
Wert
\end{tabular} & $\begin{array}{l}\text { Oberer } \\
\text { Wert }\end{array}$ \\
\hline \multirow{4}{*}{ Schritt $1^{\mathrm{a}}$} & $\begin{array}{l}\text { Auflage } \\
\text { "Auflage" }\end{array}$ & 1,082 & 0,737 & 2,155 & 1 & 0,142 & 2,951 & 0,696 & 12,511 \\
\hline & Motivationslage & & & 19,04 & 2 & 0 & & & \\
\hline & \begin{tabular}{|l|} 
Ambivalenz- \\
Motivation \\
\end{tabular} & $-2,194$ & 0,767 & 8,173 & 1 & 0,004 & 0,111 & 0,025 & 0,502 \\
\hline & Fremd-Motivation & $-4,46$ & 1,057 & $\begin{array}{l}17,81 \\
8\end{array}$ & 1 & 0 & 0,012 & 0,001 & 0,092 \\
\hline
\end{tabular}


Fortsetzung Tabelle 8: Zugang und Entlassform - Variablen in der Gleichung nach durchgeführter logistischer Regression

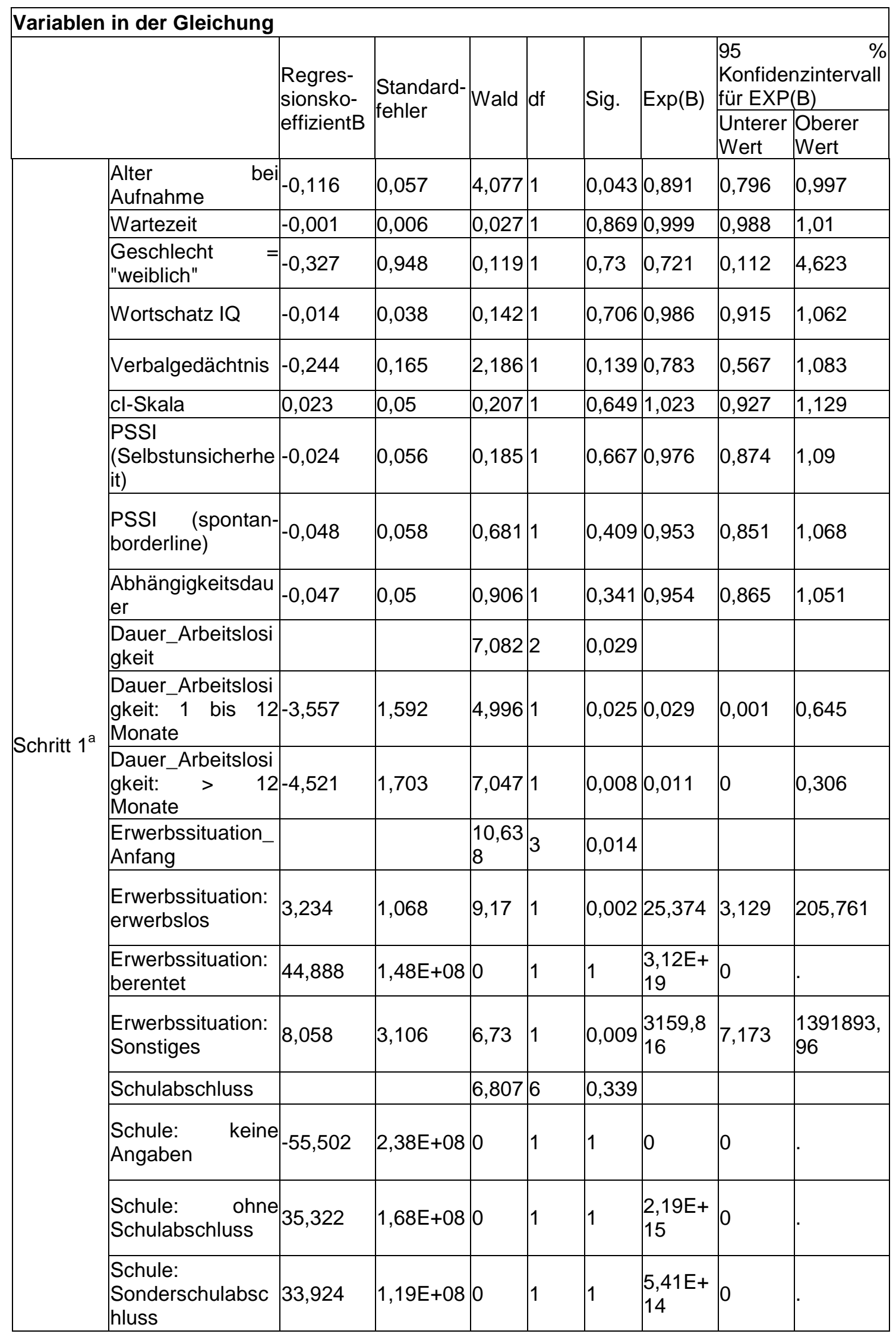


Fortsetzung Tabelle 8: Zugang und Entlassform - Variablen in der Gleichung nach durchgeführter logistischer Regression

\begin{tabular}{|c|c|c|c|c|c|c|c|c|}
\hline \multicolumn{9}{|l|}{ Variablen in der Gleichung } \\
\hline & $\begin{array}{l}\text { Regres- } \\
\text { sionsko- } \\
\text { effizientB }\end{array}$ & $\begin{array}{l}\text { Standard- } \\
\text { fehler }\end{array}$ & Wald & df & Sig. & $\operatorname{Exp}(B)$ & \multicolumn{2}{|c|}{\begin{tabular}{|l}
95 \\
Konfidenzintervall \\
für $\operatorname{EXP}(\mathrm{B})$
\end{tabular}} \\
\hline $\begin{array}{l}\text { Schule: } \\
\text { Hauptschulabschl } \\
\text { uss }\end{array}$ & $-2,569$ & 1,884 & 1,86 & 1 & 0,173 & 0,077 & 0,002 & 3,074 \\
\hline $\begin{array}{l}\text { Schule: } \\
\text { Realschule/Polyte } \\
\text { chnische } \\
\text { Oberschule }\end{array}$ & $-2,833$ & 1,771 & 2,558 & 1 & 0,11 & 0,059 & 0,002 & 1,894 \\
\hline $\begin{array}{l}\text { Schule: Anderer } \\
\text { Schulabschluss }\end{array}$ & $-8,807$ & 3,395 & 6,728 & 1 & 0,009 & 0 & 0 & 0,116 \\
\hline Kostenträger & & & 0,965 & 6 & 0,987 & & & \\
\hline $\begin{array}{l}\text { Kostenträger: } \\
\text { Andere }\end{array}$ & $-0,297$ & 2,144 & 0,019 & 1 & 0,89 & 0,743 & 0,011 & 49,649 \\
\hline $\begin{array}{l}\text { Kostenträger: } \\
\text { Andere LVAen }\end{array}$ & 37,138 & $1,93 E+08$ & 0 & 1 & 1 & $\begin{array}{l}1,35 \mathrm{E}+ \\
16\end{array}$ & 0 & \\
\hline $\begin{array}{l}\text { Kostenträger: } \\
\text { Krankenkassen }\end{array}$ & 40,574 & $1,21 \mathrm{E}+08$ & 0 & 1 & 1 & $\begin{array}{l}4,18 \mathrm{E}+ \\
17\end{array}$ & 0 & \\
\hline $\begin{array}{l}\text { Kostenträger: } \\
\text { Bahn/Knappschaft } \\
\text { /See }\end{array}$ & $t-0,302$ & 1,242 & 0,059 & 1 & 0,808 & 0,74 & 0,065 & 8,438 \\
\hline $\begin{array}{l}\text { Kostenträger: LVA } \\
\text { Sachsen-Anhalt }\end{array}$ & 0,017 & 0,912 & 0 & 1 & 0,985 & 1,017 & 0,17 & 6,072 \\
\hline $\begin{array}{l}\text { Kostenträger: LVA } \\
\text { Thüringen }\end{array}$ & 1,061 & 1,335 & 0,632 & 1 & 0,427 & 2,89 & 0,211 & 39,583 \\
\hline Familienstand & & & 7,929 & 4 & 0,094 & & & \\
\hline $\begin{array}{l}\text { Familienstand: } \\
\text { verheiratet } \\
\text { getrennt lebend }\end{array}$ & $-1,953$ & 1,64 & 1,419 & 1 & 0,233 & 0,142 & 0,006 & 3,526 \\
\hline $\begin{array}{l}\text { Familienstand: } \\
\text { ledig }\end{array}$ & $-4,482$ & 1,717 & 6,817 & 1 & 0,009 & 0,011 & 0 & 0,327 \\
\hline $\begin{array}{l}\text { Familienstand: } \\
\text { geschieden }\end{array}$ & $-2,963$ & 1,431 & 4,289 & 1 & 0,038 & 0,052 & 0,003 & 0,853 \\
\hline $\begin{array}{l}\text { Familienstand: } \\
\text { verwitwet }\end{array}$ & 30,771 & $1,67 \mathrm{E}+08$ & 0 & 1 & 1 & $\begin{array}{l}2,31 \mathrm{E}+ \\
13\end{array}$ & 0 & \\
\hline $\begin{array}{l}\text { Wiederaufnahme } \\
=\text { "Ja" (Dummy) }\end{array}$ & $-0,357$ & 0,692 & 0,266 & 1 & 0,606 & 0,7 & 0,18 & 2,717 \\
\hline
\end{tabular}


Fortsetzung Tabelle 8: Zugang und Entlassform - Variablen in der Gleichung nach durchgeführter logistischer Regression

\begin{tabular}{|c|c|c|c|c|c|c|c|c|}
\hline \multicolumn{9}{|l|}{ Variablen in der Gleichung } \\
\hline & $\begin{array}{l}\text { Regres- } \\
\text { sionsko- } \\
\text { effizientB }\end{array}$ & $\begin{array}{l}\text { Standard- } \\
\text { fehler }\end{array}$ & Wald & $d f$ & Sig. & $\operatorname{Exp}(B)$ & \multicolumn{2}{|c|}{$\begin{array}{l}95 \\
\text { Konfidenzintervall } \\
\text { für } \operatorname{EXP}(\mathrm{B})\end{array}$} \\
\hline $\begin{array}{l}\text { Zuvor } \\
\text { suchtbezogene } \\
\text { Hilfe = "Ja" } \\
\text { (Dummy) }\end{array}$ & 2,437 & 1,272 & 3,67 & 1 & 0,055 & 11,443 & 0,945 & 138,541 \\
\hline $\begin{array}{l}\text { Körperbezogene } \\
\text { Komorbidität }\end{array}$ & 0,649 & 0,893 & 0,528 & 1 & 0,467 & 1,913 & 0,333 & 11 \\
\hline \begin{tabular}{|l} 
Psychische \\
Erkrankungen
\end{tabular} & 0,808 & 1,095 & 0,545 & 1 & 0,461 & 2,244 & 0,262 & 19,207 \\
\hline \begin{tabular}{|l} 
Alkoholbezogene \\
Erkrankungen
\end{tabular} & 3,406 & 1,646 & 4,283 & 1 & 0,039 & 30,13 & 1,197 & 758,132 \\
\hline \begin{tabular}{|l} 
Zweite \\
Suchtdiagnose \\
(ohner Tabak- \\
abhängigkeit) \\
\end{tabular} & $-0,542$ & 1,278 & 0,18 & 1 & 0,671 & 0,581 & 0,047 & 7,123 \\
\hline \begin{tabular}{|l}
$\begin{array}{l}\text { Tabak- } \\
\text { abhängigkeit }\end{array}$ \\
\end{tabular} & $-0,466$ & 1,119 & 0,173 & 1 & 0,677 & 0,628 & 0,07 & 5,631 \\
\hline $\begin{array}{l}\text { Persönlichkeits- } \\
\text { störung }\end{array}$ & 0,127 & 1,226 & 0,011 & 1 & 0,918 & 1,135 & 0,103 & 12,552 \\
\hline $\begin{array}{l}\text { Intelligenz- } \\
\text { minderung }\end{array}$ & 39,995 & $1,60 \mathrm{E}+08$ & 0 & 1 & 1 & $\begin{array}{l}2,34 \mathrm{E}+ \\
17\end{array}$ & 0 & \\
\hline Konstante & 19,957 & 7,698 & 6,721 & 1 & 0,01 & $\begin{array}{l}4,65 \mathrm{E}+ \\
08\end{array}$ & & \\
\hline
\end{tabular}




\section{Zugang und Therapieergebnis}

Tabelle 9: Zugang und Therapieergebnis - Deskriptive Statistiken

Angegeben werden jeweils Mittelwert und Standardabweichung sowie mit $\mathrm{N}=230$ die Anzahl der in die linearen Regressionsanalyse einbezogenen Fälle.

\section{Legende:}

- In der ersten Zeile befindet sich die Outcome-Variable; grau hinterlegt sind die Variablen der Hypothese 1, darauf folgen die Variablen der Hypothese 2

- Als Dummy-Variablen gingen in die Berechnung ein: „Auflage“ („Auflage“ erhielt den Wert „1“), „Geschlecht“ („weiblich“ = „1“), „Wiederaufnahme“ („Ja“ = „1“), „Zuvor suchtbezogene Hilfe erhalten“ (,Ja“ = „1“)

- In Dummy-Variablen umgewandelte kategoriale Variablen sind „Motivationslage“ (Referenzkategorie (=RK) „Eigenmotiviert“), „Kostenträger“ (RK „Bundesversicherungsanstalt für Angestellte“), „Familienstand“ (RK „Verheiratet, zusammenlebend“), „Schule“ (=Schulabschluss; RK „Abitur/(Fach)Hochschulreife“), „Erwerbssituation“ (zu Therapiebeginn; RK „erwerbstätig“), „Dauer_Arbeitslosigkeit“ (RK „Arbeitslosigkeit < 1 Monat“) - die Referenzkategorien tauchen bei der multiplen linearen Regression nicht sichtbar auf, weil sie mit „, 0 “ kodiert wurden

- $\quad$ Alle anderen Variablen sind metrischer Natur

- $\quad$ „IQ“ = Intelligenzquotient, abgeschätzt anhand des Wortschatztests

- „C.I.-Skala“ = Skala zur Erfassung cerebraler Insuffizienzen

- "C-Werte“ und „T-Werte“ = geben Auskunft darüber, in welchem Verhältnis sich der Testwert eines Patienten zur Normstichprobe des Tests befindet

- $\quad$ „PSSI“ = Persönlichkeits-Stil-und-Störungs-Inventar

- $\quad$ „LVA“ = Landesversicherungsanstalt, der frühere Name der regionalen Rentenversicherungen

\begin{tabular}{|l|l|l|l|}
\hline & Mittelwert & Standardabweichung & N \\
\hline $\begin{array}{l}\text { Outcome-Variable: } \\
\text { Therapieergebnis (Rating von 1 bis 4) }\end{array}$ & 2,27 & 0,75 & 230 \\
\hline Auflage = "Auflage" & 0,7652 & 0,42479 & 230 \\
\hline Motivationslage_Ambivalenz-Motivation & 0,1783 & 0,38357 & 230 \\
\hline Motivationslage_Fremd-Motivation & 0,113 & 0,31734 & 230 \\
\hline Alter bei Aufnahme & 43,66 & 8,121 & 230 \\
\hline Wartezeit (in Tagen) & 88,26 & 64,363 & 230 \\
\hline Geschlecht 0=m, 1=w & 0,23 & 0,422 & 230 \\
\hline Wortschatz IQ & 97,33 & 10,983 & 230 \\
\hline Verbalgedächtnis (C-Werte) & 3,73 & 2,099 & 230 \\
\hline cl-Skala (Anzahl Beschwerden) & 15,05 & 8,943 & 230 \\
\hline PSSI (Selbstunsicherheit) (T-Werte) & 55,05 & 8,82 & 230 \\
\hline PSSI (spontan-borderline) (T-Werte) & 52,96 & 8,503 & 230 \\
\hline Abhängigkeitsdauer (in Jahren) & 12,62 & 7,147 & \\
\hline
\end{tabular}


Fortsetzung Tabelle 9: Zugang und Therapieergebnis - Deskriptive Statistiken

\begin{tabular}{|c|c|c|c|}
\hline & Mittelwert & Standardabweichung & $\mathrm{N}$ \\
\hline Kostenträger Andere & 0,0217 & 0,14615 & 230 \\
\hline Kostenträger_Krankenkasse & 0,0522 & 0,22286 & 230 \\
\hline Kostenträger_BahnKnappschaftSee & 0,087 & 0,28239 & 230 \\
\hline Kostenträger_LVA_Sachsen-Anhalt & 0,5391 & 0,49955 & 230 \\
\hline Kostenträger_LVA_Thüringen & 0,0435 & 0,20438 & 230 \\
\hline Kostenträger_LVA_Andere & 0,0304 & 0,17216 & 230 \\
\hline Familienstand_ledig & 0,3043 & 0,46113 & 230 \\
\hline $\begin{array}{l}\text { Familienstand_verheiratet } \quad-\quad \text { getrenn } \\
\text { lebend }\end{array}$ & 0,0696 & 0,25497 & 230 \\
\hline Familienstand_geschieden & 0,313 & 0,46474 & 230 \\
\hline Familienstand_verwitwet & 0 & 0 & 230 \\
\hline Familienstand_ohneAngaben & 0 & 0 & 230 \\
\hline Schule_keine Angaben & 0 & 0 & 230 \\
\hline Schule_ohne Schulabschluss & 0,0304 & 0,17216 & 230 \\
\hline Schule_Sonderschulabschluss & 0,0217 & 0,14615 & 230 \\
\hline Schule_Hauptschulabschluss & 0,2348 & 0,42479 & 230 \\
\hline $\begin{array}{l}\text { Schule_Realschule/Polytechnische } \\
\text { Oberschule } 10 \text {. Klasse }\end{array}$ & 0,5957 & 0,49184 & 230 \\
\hline Erwerbssituation_erwerbslos & 0,6304 & 0,48374 & 230 \\
\hline Erwerbssituation_berentet & 0,0565 & 0,23143 & 230 \\
\hline Erwerbssituation_Sonstiges & 0,0217 & 0,14615 & 230 \\
\hline Dauer_Arbeitslosigkeit: 1 bis 12 Monate & 0,213 & 0,41035 & 230 \\
\hline Dauer_Arbeitslosigkeit: > 12 Monate & 0,5043 & 0,50107 & 230 \\
\hline Wiederaufnahme = "Ja" (Dummy) & 0,2435 & 0,43012 & 230 \\
\hline $\begin{array}{l}\text { Zuvor suchtbezogene } \\
\text { Hilfe erhalten = "Ja" (Dummy) }\end{array}$ & 0,8957 & 0,30638 & 230 \\
\hline Körperbezogene Komorbidität (Anzahl) & 1,8522 & 0,84361 & 230 \\
\hline Psychische Erkrankungen (Anzahl) & 0,7 & 0,61389 & 230 \\
\hline Alkoholbezogene Erkrankungen (Anzahl) & 0,0913 & 0,28867 & 230 \\
\hline \begin{tabular}{|lll}
$\begin{array}{l}\text { Zweite Suchtdiagnose } \\
\text { abhängigkeit) (Anzahl) }\end{array}$ & (ohne & Tabak \\
\end{tabular} & 0,087 & 0,29745 & 230 \\
\hline Tabakabhängigkeit (Anzahl) & 0,6174 & 0,48708 & 230 \\
\hline Persönlichkeitsstörung (Anzahl) & 0,5696 & 0,50494 & 230 \\
\hline Intelligenzminderung (Anzahl) & 0,0174 & 0,13101 & 230 \\
\hline
\end{tabular}


Tabelle 10: Zugang und Therapieergebnis - Modellzusammenfassung der linearen Regression der im Modell zusammengefassten unabhängigen Variablen Legende:

- „R-Quadrat“ = die erklärte Varianz durch die im Modell zusammengefassten unabhängigen Variablen

- ${ } \mathrm{R}^{\prime \prime}=$ die Wurzel aus R-Quadrat, entspricht dem multiplen Korrelationskoeffizienten der abhängigen Variable mit den unabhängigen Variablen

- $\quad$ „F“ = wird bei den Änderungsstatistiken zur Berechnung des statistischen F-Tests benötigt und ergibt die Signifikanz (= „Sig.“) des Gesamtmodells

- $\quad$ „df“ = Freiheitsgrade

\begin{tabular}{|c|c|c|c|c|c|c|c|c|c|}
\hline \multicolumn{10}{|c|}{ Modellzusammenfassung } \\
\hline \multirow{3}{*}{ Modell } & \multirow[b]{2}{*}{$\mathrm{R}$} & \multirow[b]{2}{*}{$\begin{array}{l}\text { R- } \\
\text { Quadrat }\end{array}$} & \multirow[b]{2}{*}{$\begin{array}{l}\text { Korrigiertes } \\
\text { R-Quadrat }\end{array}$} & \multirow[b]{2}{*}{$\begin{array}{l}\text { Standard- } \\
\text { fehler des } \\
\text { Schätzers }\end{array}$} & \multicolumn{5}{|c|}{ Änderungsstatistiken } \\
\hline & & & & & $\begin{array}{l}\text { Änderung } \\
\text { in R R } \\
\text { Quadrat }\end{array}$ & $\begin{array}{l}\text { Änderung } \\
\text { in F }\end{array}$ & df1 & df2 & \begin{tabular}{|l|} 
Sig. \\
Änderung \\
in F
\end{tabular} \\
\hline &, 558 & 0,311 & 0,17 & 0,683 & 0,311 & 2,204 & 39 & 190 & 0,000 \\
\hline
\end{tabular}


Tabelle 11: Zugang und Therapieergebnis - Koeffizienten nach erfolgter linearer Regressionsanalyse

Legende:

- " $\mathrm{a}$ " = die abhängige Variable ist das „Therapieergebnis“

- "RegressionskoeefizientB“ = gibt die Richtung des Effekts auf das Therapieergebnis bei Erhöhung der jeweiligen unabhängigen Variable an

- "Standardisierte Koeffizienten: Beta“ = gibt an, um wie viel Standardabweichungseinheiten sich die abhängige Variable verändert, wenn die unabhängige Variabel um eine Standardabweichungseinheit erhöht wird

- $\quad$ "T“ (in der Überschrift) = wird zur Berechnung des statistischen T-Tests benötigt und ergibt die Signifikanz (= „Sig.“) des jeweiligen Effekts auf die abhängige Variable

- „95, $0 \%$ Konfidenzintervalle für $B^{\prime \prime}=$ geben jeweils an, in welchem Wertebereich sich der unstandardisierte Regressionskoeffizient B mit $95 \%$-iger Wahrscheinlichkeit befindet

- Grau hinterlegt sind die Variablen der Hypothese 1, darauf folgen die Variablen der Hypothese 2

- Als Dummy-Variablen gingen in die Berechnung ein: „Auflage“ („Auflage“ erhielt den Wert „1“), „Geschlecht“ (,weiblich“ = „1“), „Wiederaufnahme“ (,Ja“ = „1“), „Zuvor suchtbezogene Hilfe erhalten“ (,Ja“ = „1“)

- In Dummy-Variablen umgewandelte kategoriale Variablen sind „Motivationslage“ (Referenzkategorie (=RK) „Eigenmotiviert“), „Kostenträger“ (RK „Bundesversicherungsanstalt für Angestellte"), „Familienstand“ (RK „verheiratet, zusammenlebend“), „Schule“ (=Schulabschluss; RK „Abitur/(Fach)Hochschulreife“), „Erwerbssituation“ (zu Therapiebeginn; RK „erwerbstätig“), „Dauer_Arbeitslosigkeit“ (RK „Arbeitslosigkeit $<1$ Monat“) - die Referenzkategorien tauchen bei der multiplen linearen Regression nicht sichtbar auf, weil sie mit „0“ kodiert wurden

- Alle anderen Variablen sind metrischer Natur

- $\quad$ „IQ“ = Intelligenzquotient, abgeschätzt anhand des Wortschatztests

- "c.l.-Skala“ = Skala zur Erfassung cerebraler Insuffizienzen

- "C-Werte" und „T-Werte" = geben Auskunft darüber, in welchem Verhältnis sich der Testwert eines Patienten zur Normstichprobe des Tests befindet

- PSSI“ = Persönlichkeits-Stil-und-Störungs-Inventar

- $\quad$ "LVA“ = Landesversicherungsanstalt, der frühere Name der regionalen Rentenversicherungen

[Werte der Tabelle 11 beginnen der Übersichtlichkeit halber auf der folgenden Seite.] 
Fortsetzung Tabelle 11: Zugang und Therapieergebnis - Koeffizienten nach erfolgter linearer Regressionsanalyse

\begin{tabular}{|c|c|c|c|c|c|c|c|}
\hline \multicolumn{8}{|l|}{ Koeffizienten $^{a}$} \\
\hline \multirow[t]{2}{*}{ Modell } & \multicolumn{2}{|c|}{$\begin{array}{l}\text { Nicht standardisierte } \\
\text { Koeffizienten }\end{array}$} & \multirow{2}{*}{$\begin{array}{l}\text { Standard- } \\
\text { isierte } \\
\text { Koeffizien- } \\
\text { ten }\end{array}$} & \multirow[t]{2}{*}{$\mathrm{T}$} & \multirow[t]{2}{*}{ Sig. } & \multicolumn{2}{|c|}{$\begin{array}{l}95,0 \\
\text { Konfidenzintervalle } \\
\text { für B }\end{array}$} \\
\hline & $\begin{array}{l}\text { Regres- } \\
\text { sionsko- } \\
\text { effizientB }\end{array}$ & $\begin{array}{l}\text { Standard- } \\
\text { fehler }\end{array}$ & & & & $\begin{array}{l}\text { Unter- } \\
\text { grenze }\end{array}$ & $\begin{array}{l}\text { Ober- } \\
\text { grenze }\end{array}$ \\
\hline (Konstante) & 1,835 & 0,998 & & $\begin{array}{l}1,83 \\
9\end{array}$ & 0,067 & $-0,133$ & 3,804 \\
\hline Auflage = "Auflage" & $-0,068$ & 0,132 & $-0,038$ & $\begin{array}{l}- \\
0,51 \\
3\end{array}$ & 0,609 & $-0,328$ & 0,192 \\
\hline $\begin{array}{l}\text { Motivationslage_Ambi } \\
\text { valenz-Motivation }\end{array}$ & 0,112 & 0,139 & 0,057 & $\begin{array}{l}0,80 \\
6\end{array}$ & 0,421 & $-0,163$ & 0,387 \\
\hline $\begin{array}{l}\text { Motivationslage_Frem } \\
\text { d-Motivation }\end{array}$ & 0,436 & 0,176 & 0,185 & 2,48 & 0,014 & 0,089 & 0,783 \\
\hline Alter bei Aufnahme & 0,008 & 0,008 & 0,084 & $\begin{array}{l}0,96 \\
9\end{array}$ & 0,334 & $-0,008$ & 0,024 \\
\hline Wartezeit (in Tagen) & $-0,001$ & 0,001 & $-0,085$ & $\begin{array}{l}- \\
1,14 \\
5\end{array}$ & 0,254 & $-0,003$ & 0,001 \\
\hline Geschlecht $0=m, 1=w$ & $-0,37$ & 0,142 & $-0,208$ & $\begin{array}{l}- \\
2,60 \\
9\end{array}$ & 0,010 & $-0,649$ & $-0,09$ \\
\hline Wortschatz IQ & $-0,006$ & 0,006 & $-0,087$ & $\begin{array}{l}0,99 \\
7\end{array}$ & 0,320 & $-0,018$ & 0,006 \\
\hline Verbalgedächtnis & 0,021 & 0,026 & 0,059 & $\begin{array}{l}0,80 \\
4\end{array}$ & 0,422 & $-0,03$ & 0,072 \\
\hline cl-Skala & $-0,008$ & 0,007 & $-0,096$ & $\begin{array}{l}- \\
1,09 \\
2\end{array}$ & 0,276 & $-0,023$ & 0,007 \\
\hline $\begin{array}{l}\text { PSSI } \\
\text { (Selbstunsicherheit) }\end{array}$ & $-0,008$ & 0,008 & $-0,088$ & $\begin{array}{l}- \\
0,91 \\
5\end{array}$ & 0,362 & $-0,024$ & 0,009 \\
\hline \begin{tabular}{|ll} 
PSSI & (spontan- \\
borderline) &
\end{tabular} & 0,011 & 0,009 & 0,129 & 1,3 & 0,195 & $-0,006$ & 0,029 \\
\hline \begin{tabular}{|l}
$\begin{array}{l}\text { Abhängigkeitsdauer } \\
\text { (in Jahren) }\end{array}$ \\
\end{tabular} & 0,019 & 0,008 & 0,181 & $\begin{array}{l}2,50 \\
6 \\
\end{array}$ & 0,013 & 0,004 & 0,034 \\
\hline Kostenträger_Andere & $-0,266$ & 0,348 & $-0,052$ & $\begin{array}{l}- \\
0,76 \\
3 \\
\end{array}$ & 0,446 & $-0,952$ & 0,421 \\
\hline $\begin{array}{l}\text { Kostenträger_Kranken } \\
\text { kasse }\end{array}$ & $-0,002$ & 0,263 & $-0,001$ & $\begin{array}{l}- \\
0,00 \\
8\end{array}$ & 0,994 & $-0,52$ & 0,516 \\
\hline $\begin{array}{l}\text { Kostenträger_BahnKn } \\
\text { appschaftSee }\end{array}$ & $-0,008$ & 0,207 & $-0,003$ & $\begin{array}{l}- \\
0,03 \\
7\end{array}$ & 0,971 & $-0,417$ & 0,401 \\
\hline
\end{tabular}


Fortsetzung Tabelle 11: Zugang und Therapieergebnis - Koeffizienten nach erfolgter linearer Regressionsanalyse

\begin{tabular}{|c|c|c|c|c|c|c|c|}
\hline \multicolumn{8}{|l|}{ Koeffizienten $^{\mathrm{a}}$} \\
\hline \multirow{2}{*}{ Modell } & \multicolumn{2}{|c|}{$\begin{array}{l}\text { Nicht standardisiert } \\
\text { Koeffizienten }\end{array}$} & \multirow{2}{*}{\begin{tabular}{|l}
$\begin{array}{l}\text { Standard- } \\
\text { eisierte } \\
\text { Koeffizien- } \\
\text { ten }\end{array}$ \\
Beta
\end{tabular}} & \multirow{2}{*}{$\mathrm{T}$} & \multirow{2}{*}{ Sig. } & \multicolumn{2}{|c|}{$\begin{array}{l}95,0 \quad \% \\
\text { Konfidenzintervalle } \\
\text { für B }\end{array}$} \\
\hline & $\begin{array}{l}\text { Regres- } \\
\text { sionsko- } \\
\text { effizientB }\end{array}$ & $\begin{array}{l}\text { Standard- } \\
\text { fehler }\end{array}$ & & & & $\begin{array}{l}\text { Unter- } \\
\text { grenze }\end{array}$ & $\begin{array}{l}\text { Ober- } \\
\text { grenze }\end{array}$ \\
\hline $\begin{array}{l}\text { Kostenträger_LVA_Sa } \\
\text { chsen-Anhalt }\end{array}$ & $-0,036$ & 0,14 & $-0,024$ & $\begin{array}{l}- \\
0,26 \\
1\end{array}$ & 0,795 & $-0,312$ & 0,239 \\
\hline $\begin{array}{l}\begin{array}{l}\text { Kostenträger_LVA_Th } \\
\text { üringen }\end{array} \\
\end{array}$ & 0,168 & 0,264 & 0,046 & $\begin{array}{l}0,63 \\
7\end{array}$ & 0,525 & $-0,352$ & 0,689 \\
\hline $\begin{array}{l}\text { Kostenträger_LVA_An } \\
\text { dere }\end{array}$ & $-0,338$ & 0,303 & $-0,078$ & $\begin{array}{l}1,11 \\
4\end{array}$ & 0,267 & $-0,937$ & 0,26 \\
\hline Familienstand_ledig & 0,273 & 0,156 & 0,168 & $\begin{array}{l}1,74 \\
9\end{array}$ & 0,082 & $-0,035$ & 0,581 \\
\hline $\begin{array}{l}\text { Familienstand_verheir } \\
\text { atet - getrennt lebend }\end{array}$ & $-0,052$ & 0,211 & $-0,018$ & $\begin{array}{l}- \\
0,24 \\
8\end{array}$ & 0,805 & $-0,468$ & 0,363 \\
\hline $\begin{array}{l}\text { Familienstand_geschie } \\
\text { den }\end{array}$ & 0,116 & 0,144 & 0,072 & $\begin{array}{l}0,80 \\
4\end{array}$ & 0,422 & $-0,168$ & 0,399 \\
\hline $\begin{array}{l}\text { Schule_ohne } \\
\text { Schulabschluss }\end{array}$ & 0,078 & 0,346 & 0,018 & $\begin{array}{l}0,22 \\
4\end{array}$ & 0,823 & $-0,606$ & 0,761 \\
\hline $\begin{array}{l}\text { Schule_Sonderschulab } \\
\text { schluss }\end{array}$ & 0,777 & 0,44 & 0,151 & $\begin{array}{l}1,76 \\
7\end{array}$ & 0,079 & $-0,09$ & 1,644 \\
\hline $\begin{array}{l}\text { Schule_Hauptschulabs } \\
\text { chluss }\end{array}$ & 0,256 & 0,209 & 0,145 & $\begin{array}{l}1,22 \\
5\end{array}$ & 0,222 & $-0,156$ & 0,669 \\
\hline $\begin{array}{l}\text { Schule_Realschule/Pol } \\
\text { ytechnische } \\
\text { Oberschule 10. Klasse }\end{array}$ & 0,129 & 0,166 & 0,085 & $\begin{array}{l}0,77 \\
9\end{array}$ & 0,437 & $-0,198$ & 0,457 \\
\hline \begin{tabular}{|l|}
$\begin{array}{l}\text { Erwerbssituation_erwe } \\
\text { rbslos }\end{array}$ \\
\end{tabular} & 0,061 & 0,174 & 0,039 & $\begin{array}{l}0,35 \\
1\end{array}$ & 0,726 & $-0,282$ & 0,404 \\
\hline $\begin{array}{l}\text { Erwerbssituation_bere } \\
\text { ntet }\end{array}$ & $-0,322$ & 0,25 & $-0,099$ & $\begin{array}{l}1,29 \\
1\end{array}$ & 0,198 & $-0,815$ & 0,17 \\
\hline $\begin{array}{l}\text { Erwerbssituation_Sons } \\
\text { tiges }\end{array}$ & 0,24 & 0,369 & 0,047 & 0,65 & 0,516 & $-0,488$ & 0,968 \\
\hline $\begin{array}{l}\text { Dauer_Arbeitslosigkeit: } \\
1 \text { bis } 12 \text { Monate }\end{array}$ & 0,029 & 0,178 & 0,016 & $\begin{array}{l}0,16 \\
2\end{array}$ & 0,872 & $-0,322$ & 0,38 \\
\hline $\begin{array}{l}\text { Dauer_Arbeitslosigkeit: } \\
>12 \text { Monate }\end{array}$ & 0,077 & 0,192 & 0,051 & $\begin{array}{l}0,40 \\
2\end{array}$ & 0,688 & $-0,301$ & 0,455 \\
\hline $\begin{array}{l}\text { Wiederaufnahme } \\
\text { "Ja" }\end{array}$ & $=-0,026$ & 0,117 & $-0,015$ & $\begin{array}{l}0,21 \\
9\end{array}$ & 0,827 & $-0,255$ & 0,204 \\
\hline $\begin{array}{l}\text { Zuvor suchtbezogene } \\
\text { Hilfe erhalten = "Ja" }\end{array}$ & 0,001 & 0,165 & 0,001 & $\begin{array}{l}0,00 \\
8\end{array}$ & 0,994 & $-0,324$ & 0,326 \\
\hline
\end{tabular}


Fortsetzung Tabelle 11: Zugang und Therapieergebnis - Koeffizienten nach erfolgter linearer Regressionsanalyse

\begin{tabular}{|c|c|c|c|c|c|c|c|}
\hline \multicolumn{8}{|l|}{ Koeffizienten $^{a}$} \\
\hline \multirow{2}{*}{ Modell } & \multicolumn{2}{|c|}{$\begin{array}{l}\text { Nicht standardisiert } \\
\text { Koeffizienten }\end{array}$} & \multirow{2}{*}{\begin{tabular}{|l}
$\begin{array}{l}\text { Standard- } \\
\text { isierte } \\
\text { Koeffizien- } \\
\text { ten }\end{array}$ \\
Beta
\end{tabular}} & \multirow{2}{*}{$\mathrm{T}$} & \multirow{2}{*}{ Sig. } & \multicolumn{2}{|c|}{$\begin{array}{l}95,0 \quad \% \\
\text { Konfidenzintervalle } \\
\text { für B }\end{array}$} \\
\hline & \begin{tabular}{|l|} 
Regres- \\
sionsko- \\
effizientB
\end{tabular} & $\begin{array}{l}\text { Standard- } \\
\text { fehler }\end{array}$ & & & & $\begin{array}{l}\text { Unter- } \\
\text { grenze }\end{array}$ & $\begin{array}{l}\text { Ober- } \\
\text { grenze }\end{array}$ \\
\hline $\begin{array}{l}\text { Körperbezogene } \\
\text { Komorbidität }\end{array}$ & $-0,035$ & 0,167 & $-0,039$ & $\begin{array}{l}0,20 \\
7\end{array}$ & 0,837 & $-0,365$ & 0,296 \\
\hline $\begin{array}{l}\text { Psychische } \\
\text { Erkrankungen }\end{array}$ & 0,131 & 0,188 & 0,107 & $\begin{array}{l}0,69 \\
9\end{array}$ & 0,485 & $-0,239$ & 0,502 \\
\hline $\begin{array}{l}\text { Alkoholbezogene } \\
\text { Erkrankungen }\end{array}$ & $-0,064$ & 0,235 & $-0,025$ & $\begin{array}{l}0,27 \\
3\end{array}$ & 0,785 & $-0,527$ & 0,399 \\
\hline $\begin{array}{ll}\text { Zweite } & \text { Suchtdiagnose } \\
\text { (ohne } & \text { Tabak- } \\
\text { abhängigkeit) } & \\
\end{array}$ & $-0,397$ & 0,229 & 0,157 & $\begin{array}{l}1,73 \\
4\end{array}$ & 0,085 & $-0,055$ & 0,848 \\
\hline Tabakabhängigkeit & 0,021 & 0,191 & 0,013 & $\begin{array}{l}0,10 \\
9\end{array}$ & 0,914 & $-0,356$ & 0,397 \\
\hline Persönlichkeitsstörung & 0,106 & 0,203 & 0,072 & $\begin{array}{l}0,52 \\
4\end{array}$ & 0,601 & $-0,294$ & 0,506 \\
\hline Intelligenzminderung & $-0,233$ & 0,455 & $-0,041$ & $\begin{array}{l}0,51 \\
3\end{array}$ & 0,609 & $-1,13$ & 0,664 \\
\hline
\end{tabular}




\section{Zugang und Leistungsbild}

Tabelle 12: Zugang und Leistungsbild - Deskriptive Statistiken

Angegeben werden jeweils Mittelwert und Standardabweichung sowie mit $\mathrm{N}=259$ die Anzahl der in die linearen Regressionsanalyse einbezogenen Fälle.

\section{Legende:}

- In der ersten Zeile befindet sich die Outcome-Variable; grau hinterlegt sind die Variablen der Hypothese 1, darauf folgen die Variablen der Hypothese 2

- Als Dummy-Variablen gingen in die Berechnung ein: „Auflage“ (Auflage“ erhielt den Wert „1“), "Geschlecht“ (,weiblich“ = „1“), „Wiederaufnahme“ (,Ja“ = „1“), „Zuvor suchtbezogene Hilfe erhalten“ („Ja“ = „1“)

- In Dummy-Variablen umgewandelte kategoriale Variablen sind „Motivationslage“ (Referenzkategorie (=RK) „Eigenmotiviert"), „Kostenträger“ (RK „Bundesversicherungsanstalt für Angestellte"), „Familienstand“ (RK „verheiratet, zusammenlebend“), „Schule“ (=Schulabschluss; RK „Abitur/(Fach)Hochschulreife“), „Erwerbssituation“ (zu Therapiebeginn; RK „erwerbstätig“), „Dauer_Arbeitslosigkeit“ (RK „Arbeitslosigkeit < 1 Monat“) - die Referenzkategorien tauchen bei der multiplen linearen Regression nicht sichtbar auf, weil sie mit „0“ kodiert wurden

- $\quad$ Alle anderen Variablen sind metrischer Natur

- $\quad$ „IQ“ = Intelligenzquotient, abgeschätzt anhand des Wortschatztests

- "c.l.-Skala“ = Skala zur Erfassung cerebraler Insuffizienzen

- "C-Werte“ und „T-Werte" = geben Auskunft darüber, in welchem Verhältnis sich der Testwert eines Patienten zur Normstichprobe des Tests befindet

- „PSSI“ = Persönlichkeits-Stil-und-Störungs-Inventar

- $\quad$ "LVA“ = Landesversicherungsanstalt, der frühere Name der regionalen Rentenversicherungen

\begin{tabular}{|l|l|l|l|}
\hline & Mittelwert & Standardabweichung & N \\
\hline $\begin{array}{l}\text { Leistungsbild für allgemeinen } \\
\text { Arbeitsmarkt }\end{array}$ & 5,2703 & 0,63819 & 259 \\
\hline Auflage= "Auflage" & 0,7568 & 0,42987 & 259 \\
\hline Motivationslage_Ambivalenz-Motivation & 0,1737 & 0,37962 & 259 \\
\hline Motivationslage_Fremd-Motivation & 0,1274 & 0,33408 & 259 \\
\hline
\end{tabular}


Fortsetzung Tabelle 12: Zugang und Leistungsbild - Deskriptive Statistiken

\begin{tabular}{|c|c|c|c|}
\hline & Mittelwert & Standardabweichung & $\mathrm{N}$ \\
\hline Alter bei Aufnahme & 43,2 & 7,882 & 259 \\
\hline Wartezeit (in Tagen) & 90,22 & 63,357 & 259 \\
\hline Geschlecht $0=m, 1=w$ & 0,22 & 0,418 & 259 \\
\hline Wortschatz IQ & 96,81 & 10,899 & 259 \\
\hline Verbalgedächtnis (C-Werte) & 3,75 & 2,142 & 259 \\
\hline cl-Skala (Anzahl Beschwerden) & 15,08 & 8,83 & 259 \\
\hline PSSI (Selbstunsicherheit) (T-Werte) & 55,23 & 8,765 & 259 \\
\hline PSSI (spontan-borderline) (T-Werte) & 53,07 & 8,415 & 259 \\
\hline Abhängigkeitsdauer (in Jahren) & 12,88 & 8,686 & 259 \\
\hline Kostenträger_Andere & 0,0193 & 0,13786 & 259 \\
\hline Kostenträger_Krankenkasse & 0,027 & 0,16248 & 259 \\
\hline Kostenträger_BahnKnappschaftSee & 0,0965 & 0,29588 & 259 \\
\hline Kostenträger_LVA_Sachsen-Anhalt & 0,556 & 0,49782 & 259 \\
\hline Kostenträger_LVA_Thüringen & 0,0541 & 0,22656 & 259 \\
\hline Kostenträger_LVA_Andere & 0,027 & 0,16248 & 259 \\
\hline Familienstand_ledig & 0,3205 & 0,46756 & 259 \\
\hline $\begin{array}{l}\text { Familienstand_verheiratet }- \text { getrennt } \\
\text { lebend }\end{array}$ & 0,0772 & 0,26746 & 259 \\
\hline Familienstand_geschieden & 0,3012 & 0,45965 & 259 \\
\hline Familienstand_verwitwet & 0,0077 & 0,0877 & 259 \\
\hline Familienstand_ohneAngaben & 0 & 0 & 259 \\
\hline Schule_keine Angaben & 0 & 0 & 259 \\
\hline Schule_ohne Schulabschluss & 0,0309 & 0,17335 & 259 \\
\hline Schule_Sonderschulabschluss & 0,027 & 0,16248 & 259 \\
\hline Schule_Hauptschulabschluss & 0,2239 & 0,41769 & 259 \\
\hline $\begin{array}{l}\text { Schule_Realschule/Polytechnische } \\
\text { Oberschule 10. Klasse }\end{array}$ & 0,5946 & 0,49192 & 259 \\
\hline Erwerbssituation_erwerbslos & 0,6486 & 0,47832 & 259 \\
\hline Erwerbssituation_berentet & 0,0309 & 0,17335 & 259 \\
\hline Erwerbssituation_Sonstiges & 0,0232 & 0,15072 & 259 \\
\hline
\end{tabular}

Fortsetzung Tabelle 12: Zugang und Leistungsbild - Deskriptive Statistiken 


\begin{tabular}{|l|l|l|l|}
\hline & Mittelwert & Standardabweichung & N \\
\hline Dauer_Arbeitslosigkeit: 1 bis 12 Monate & 0,2201 & 0,4151 & 259 \\
\hline Dauer_Arbeitslosigkeit: > 12 Monate & 0,5135 & 0,50079 & 259 \\
\hline Wiederaufnahme = "Ja" (Dummy) & 0,2896 & 0,45444 & 259 \\
\hline $\begin{array}{l}\text { Zuvor suchtbezogene } \\
\text { Hilfe erhalten = "Ja" (Dummy) }\end{array}$ & 0,8764 & 0,32971 & 259 \\
\hline Körperbezogene Komorbidität (Anzahl) & 1,8456 & 0,81607 & 259 \\
\hline Psychische Erkrankungen (Anzahl) & 0,6795 & 0,60489 & 259 \\
\hline Alkoholbezogene Erkrankungen (Anzahl) & 0,0927 & 0,29052 & 259 \\
\hline $\begin{array}{l}\text { Zweite Suchtdiagnose (ohne Tabak- } \\
\text { abhängigkeit) (Anzahl) }\end{array}$ & 0,0888 & 0,2983 & 259 \\
\hline Tabakabhängigkeit (Anzahl) & 0,6409 & 0,48066 & 259 \\
\hline Persönlichkeitsstörung (Anzahl) & 0,5676 & 0,50412 & 259 \\
\hline Intelligenzminderung (Anzahl) & 0,0232 & 0,15072 & 259 \\
\hline
\end{tabular}


Tabelle 13: Zugang und Leistungsbild - Modellzusammenfassung der linearen Regression der im Modell zusammengefassten unabhängigen Variablen

\section{Legende:}

- "R-Quadrat" $=$ die erklärte Varianz durch die im Modell zusammengefassten unabhängigen Variablen

- „R“ = die Wurzel aus R-Quadrat, entspricht dem multiplen Korrelationskoeffizienten der abhängigen Variable mit den unabhängigen Variablen

- „F“ = wird bei den Änderungsstatistiken zur Berechnung des statistischen F-Tests benötigt und ergibt die Signifikanz (= „Sig.") des Gesamtmodells

- $\quad$ "df" = Freiheitsgrade

\section{Modellzusammenfassung}

\begin{tabular}{|c|c|c|c|c|c|c|c|c|c|}
\hline \multirow{3}{*}{ Modell } & \multirow[b]{2}{*}{ R } & \multirow[b]{2}{*}{$\begin{array}{l}\text { R- } \\
\text { Quadrat }\end{array}$} & \multirow[b]{2}{*}{$\begin{array}{l}\text { Korrigiertes } \\
\text { R-Quadrat }\end{array}$} & \multirow[b]{2}{*}{$\begin{array}{l}\text { Standard- } \\
\text { fehler des } \\
\text { Schätzers }\end{array}$} & \multicolumn{5}{|c|}{ Änderungsstatistiken } \\
\hline & & & & & $\begin{array}{l}\text { Änderun } \\
\text { in } \\
\text { Quadrat }\end{array}$ & $\begin{array}{l}\mathrm{g} \text {-Änderung } \\
\text { in F }\end{array}$ & df1 & df2 & $\begin{array}{l}\text { Sig. } \\
\text { Änderung } \\
\text { in F }\end{array}$ \\
\hline & .563 & 0,317 & 0,192 & 0,5736 & 0,317 & 2,535 & 40 & 218 & 0,000 \\
\hline
\end{tabular}


Tabelle 14: Zugang und Leistungsbild - Koeffizienten nach erfolgter linearer Regressionsanalyse

Legende:

- $\quad$ "a“ = die abhängige Variable ist das „Therapieergebnis“

- "RegressionskoeefizientB“ = gibt die Richtung des Effekts auf das Therapieergebnis bei Erhöhung der jeweiligen unabhängigen Variable an

- "Standardisierte Koeffizienten: Beta“ = gibt an, um wie viel Standardabweichungseinheiten sich die abhängige Variable verändert, wenn die unabhängige Variabel um eine Standardabweichungseinheit erhöht wird

- $\quad$ "T“ (in der Überschrift) = wird zur Berechnung des statistischen T-Tests benötigt und ergibt die Signifikanz (= „Sig.“) des jeweiligen Effekts auf die abhängige Variable

- „95, $0 \%$ Konfidenzintervalle für $\mathrm{B}^{\prime \prime}=$ geben jeweils an, in welchem Wertebereich sich der unstandardisierte Regressionskoeffizient B mit $95 \%$-iger Wahrscheinlichkeit befindet

- Grau hinterlegt sind die Variablen der Hypothese 1, darauf folgen die Variablen der Hypothese 2

- Als Dummy-Variablen gingen in die Berechnung ein: „Auflage“ („Auflage“ erhielt den Wert „1“), „Geschlecht“ (,weiblich“ = „1“), „Wiederaufnahme“ (,Ja“ = „1“), „Zuvor suchtbezogene Hilfe erhalten“ (,Ja“ = „1“)

- In Dummy-Variablen umgewandelte kategoriale Variablen sind „Motivationslage“ (Referenzkategorie (=RK) „Eigenmotiviert“), „Kostenträger“ (RK „Bundesversicherungsanstalt für Angestellte"), „Familienstand“ (RK „verheiratet, zusammenlebend“), „Schule“ (=Schulabschluss; RK „Abitur/(Fach)Hochschulreife"), „Erwerbssituation“ (zu Therapiebeginn; RK „erwerbstätig“), „Dauer_Arbeitslosigkeit“ (RK „Arbeitslosigkeit $<1$ Monat“) - die Referenzkategorien tauchen bei der multiplen linearen Regression nicht sichtbar auf, weil sie mit „0“ kodiert wurden

- Alle anderen Variablen sind metrischer Natur

- $\quad$ „IQ“ = Intelligenzquotient, abgeschätzt anhand des Wortschatztests

- "C.I.-Skala“ = Skala zur Erfassung cerebraler Insuffizienzen

- "C-Werte“ und „T-Werte“ = geben Auskunft darüber, in welchem Verhältnis sich der Testwert eines Patienten zur Normstichprobe des Tests befindet

- PSSI“ = Persönlichkeits-Stil-und-Störungs-Inventar

- $\quad$ "LVA“ = Landesversicherungsanstalt, der frühere Name der regionalen Rentenversicherungen

[Werte der Tabelle 14 beginnen der Übersichtlichkeit halber auf der folgenden Seite.] 
Fortsetzung Tabelle 14: Zugang und Leistungsbild - Koeffizienten nach erfolgter linearer Regressionsanalyse

\begin{tabular}{|c|c|c|c|c|c|c|c|}
\hline \multicolumn{8}{|l|}{ Koeffizienten $^{\mathrm{a}}$} \\
\hline \multirow{2}{*}{ Modell } & \multicolumn{2}{|c|}{$\begin{array}{l}\text { Nicht standardisierte } \\
\text { Koeffizienten }\end{array}$} & \multirow{2}{*}{\begin{tabular}{|l|l} 
Standardisierte \\
Koeffizienten
\end{tabular}} & \multirow{2}{*}{$T$} & \multirow{2}{*}{ Sig. } & \multicolumn{2}{|c|}{$\begin{array}{l}95,0 \\
\text { Konfidenzintervalle } \\
\text { für B }\end{array}$} \\
\hline & $\begin{array}{l}\text { Regressions- } \\
\text { koeffizientB }\end{array}$ & $\begin{array}{l}\text { Standard- } \\
\text { fehler }\end{array}$ & & & & $\begin{array}{l}\text { Unter- } \\
\text { grenze }\end{array}$ & $\begin{array}{l}\text { Ober- } \\
\text { grenze }\end{array}$ \\
\hline (Konstante) & 3,973 & 0,82 & & 4,847 & 0,000 & 2,357 & 5,588 \\
\hline Auflage = "Auflage" & $-0,012$ & 0,102 & $-0,008$ & 0,115 & 0,908 & $-0,212$ & 0,189 \\
\hline $\begin{array}{l}\text { Motivationslage_Ambivalenz- } \\
\text { Motivation }\end{array}$ & 0,134 & 0,11 & 0,08 & 1,216 & 0,225 & $-0,083$ & 0,352 \\
\hline $\begin{array}{l}\text { Motivationslage_Fremd- } \\
\text { Motivation }\end{array}$ & 0,102 & 0,131 & 0,053 & 0,778 & 0,437 & $-0,156$ & 0,36 \\
\hline Alter bei Aufnahme & 0,005 & 0,006 & 0,066 & 0,847 & 0,398 & $-0,007$ & 0,018 \\
\hline Wartezeit (in Tagen) & 0 & 0,001 & 0,049 & 0,737 & 0,462 & $-0,001$ & 0,002 \\
\hline Geschlecht $0=m, 1=w$ & 0,039 & 0,111 & 0,025 & 0,348 & 0,728 & $-0,181$ & 0,258 \\
\hline Wortschatz IQ & 0,002 & 0,005 & 0,04 & 0,482 & 0,630 & $-0,007$ & 0,012 \\
\hline Verbalgedächtnis & $-0,043$ & 0,02 & $-0,144$ & 2,118 & 0,035 & $-0,083$ & $-0,003$ \\
\hline cl-Skala & $-0,003$ & 0,006 & $-0,042$ & 0,515 & 0,607 & $-0,015$ & 0,009 \\
\hline PSSI (Selbstunsicherheit) & 0,005 & 0,007 & 0,069 & 0,766 & 0,444 & $-0,008$ & 0,018 \\
\hline PSSI (spontan-borderline) & $-0,001$ & 0,007 & $-0,011$ & 0,115 & 0,909 & $-0,015$ & 0,013 \\
\hline $\begin{array}{l}\text { Abhängigkeitsdauer } \\
\text { Jahren) }\end{array}$ & $-0,002$ & 0,005 & $-0,031$ & 0,483 & 0,630 & $-0,012$ & 0,007 \\
\hline Kostenträger_Andere & 0,394 & 0,289 & 0,085 & 1,362 & 0,175 & $-0,176$ & 0,963 \\
\hline Kostenträger_Krankenkasse & 0,333 & 0,25 & 0,085 & 1,332 & 0,184 & $-0,16$ & 0,826 \\
\hline \begin{tabular}{|l} 
Kostenträger_Bahn \\
KnappschaftSee
\end{tabular} & 0,034 & 0,154 & 0,016 & 0,219 & 0,827 & $-0,269$ & 0,337 \\
\hline \begin{tabular}{|l|}
$\begin{array}{l}\text { Kostenträger_LVA_Sachsen- } \\
\text { Anhalt }\end{array}$ \\
\end{tabular} & 0,123 & 0,112 & 0,096 & 1,1 & 0,272 & $-0,098$ & 0,345 \\
\hline Kostenträger_LVA_Thüringen. & $-0,01$ & 0,189 & $-0,003$ & 0,051 & 0,960 & $-0,382$ & 0,363 \\
\hline Kostenträger_LVA_Andere & 0,037 & 0,248 & 0,009 & 0,148 & 0,882 & $-0,452$ & 0,525 \\
\hline Familienstand_ledig & $-0,218$ & 0,125 & $-0,16$ & $-1,748$ & 0,082 & $-0,464$ & 0,028 \\
\hline $\begin{array}{l}\text { Familienstand_verheiratet } \\
\text { getrennt lebend }\end{array}$ & $-0,218$ & 0,16 & $-0,092$ & - & 0,173 & $-0,533$ & 0,097 \\
\hline Familienstand_geschieden & $-0,062$ & 0,116 & $-0,045$ & 0,536 & 0,592 & $-0,292$ & 0,167 \\
\hline Familienstand_verwitwet & $-0,491$ & 0,468 & $-0,068$ & $-1,05$ & 0,295 & $-1,413$ & 0,431 \\
\hline
\end{tabular}


Fortsetzung Tabelle 14: Zugang und Leistungsbild - Koeffizienten nach erfolgter linearer Regressionsanalyse

\begin{tabular}{|c|c|c|c|c|c|c|c|}
\hline \multicolumn{8}{|l|}{ Koeffizienten $^{a}$} \\
\hline \multirow{2}{*}{ Modell } & \multicolumn{2}{|c|}{$\begin{array}{l}\text { Nicht standardisierte } \\
\text { Koeffizienten }\end{array}$} & \multirow{2}{*}{\begin{tabular}{|l} 
Standardisierte \\
Koeffizienten
\end{tabular}} & \multirow{2}{*}{$T$} & \multirow[t]{2}{*}{ Sig. } & \multicolumn{2}{|c|}{$\begin{array}{l}95,0 \\
\text { Konfidenzintervalle } \\
\text { für B }\end{array}$} \\
\hline & $\begin{array}{l}\text { Regressions } \\
\text { koeffizientB }\end{array}$ & $\begin{array}{l}\text { Standard- } \\
\text { fehler }\end{array}$ & & & & $\begin{array}{l}\text { Unter- } \\
\text { grenze }\end{array}$ & \begin{tabular}{|l|} 
Ober- \\
grenze
\end{tabular} \\
\hline Schule_ohne Schulabschluss & 0,214 & 0,261 & 0,058 & 0,82 & 0,413 - & $-0,301$ & 0,729 \\
\hline Schule_Sonderschulabschluss & 0,289 & 0,325 & 0,074 & 0,891 & 0,374 & $-0,351$ & 0,929 \\
\hline Schule_Hauptschulabschluss & 0,409 & 0,164 & 0,268 & 2,497 & $0,013 \mid c$ & 0,086 & 0,733 \\
\hline $\begin{array}{l}\text { Schule_Realschule/Polytechnische } \\
\text { Oberschule 10. Klasse }\end{array}$ & 0,19 & 0,129 & 0,147 & 1,479 & 0,141 & $-0,063$ & 0,444 \\
\hline Erwerbssituation_erwerbslos & 0,243 & 0,138 & 0,182 & 1,767 & 0,079 & $-0,028$ & 0,515 \\
\hline Erwerbssituation_berentet & 1,043 & 0,234 & 0,283 & 4,449 & $|0,000| c$ & 0,581 & 1,505 \\
\hline Erwerbssituation_Sonstiges & 0,551 & 0,28 & 0,13 & 1,97 & 0,050 & 0 & 1,103 \\
\hline $\begin{array}{l}\text { Dauer_Arbeitslosigkeit: } 1 \text { bis } 12 \\
\text { Monate }\end{array}$ & $-0,064$ & 0,143 & $-0,042$ & 0,449 & $0,654 \mid$ & $-0,346$ & 0,218 \\
\hline \begin{tabular}{|lll}
$\begin{array}{l}\text { Dauer_Arbeitslosigkeit: } \\
\text { Monate }\end{array}$ & $>12$ \\
\end{tabular} & $-0,136$ & 0,156 & $-0,107$ & $\overline{0}, 873$ & 0,384 & $-0,443$ & 0,171 \\
\hline Wiederaufnahme = "Ja" & 0,015 & 0,087 & 0,011 & 0,177 & 0,860 & $-0,157$ & 0,188 \\
\hline $\begin{array}{l}\text { Zuvor suchtbezogene Hilfe } \\
\text { erhalten = "Ja" }\end{array}$ & $-0,195$ & 0,123 & $-0,101$ & $\overline{1}-577$ & 0,116 & $-0,438$ & 0,049 \\
\hline Körperbezogene Komorbidität & 0,234 & 0,136 & 0,3 & 1,719 & 0,087 & $-0,034$ & 0,503 \\
\hline Psychische Erkrankungen & 0,209 & 0,153 & 0,198 & $1,367 \mathrm{C}$ & 0,173 & $-0,092$ & 0,51 \\
\hline Alkoholbezogene Erkrankungen & 0,362 & 0,191 & 0,165 & 1,893 & 0,060 & $-0,015$ & 0,739 \\
\hline $\begin{array}{ll}\text { Zweite Suchtdiagnose } & \text { (ohne } \\
\text { Tabak-abhängigkeit) } & \\
\end{array}$ & 0,365 & 0,186 & 0,171 & 1,969 & $0,050 \mid c$ & 0 & 0,731 \\
\hline Tabakabhängigkeit & $-0,048$ & 0,154 & $-0,036$ & $\overline{0}, 312$ & 0,755 & $-0,351$ & 0,255 \\
\hline Persönlichkeitsstörung & $-0,014$ & 0,163 & $-0,011$ & 0,085 & 0,932 & $-0,335$ & 0,307 \\
\hline Intelligenzminderung & 0,49 & 0,328 & 0,116 & 1,493 & 0,137 & $-0,157$ & 1,136 \\
\hline
\end{tabular}




\section{Zugang und Abstinenzrate}

Tabelle 15: Zugang und Abstinenzrate - Zusammenfassung der Fallverarbeitung der logistischen Regression

\begin{tabular}{|c|c|c|c|}
\hline \multicolumn{4}{|c|}{ Zusammenfassung der Fallverarbeitung } \\
\hline & & $\mathrm{N}$ & Prozent \\
\hline \multirow{3}{*}{$\begin{array}{l}\text { Ausgewählte } \\
\text { Fälle }\end{array}$} & $\begin{array}{l}\text { Einbezogen in } \\
\text { Analyse }\end{array}$ & 265 & 61,1 \\
\hline & \begin{tabular}{|l} 
Fehlende \\
Fälle
\end{tabular} & 169 & 38,9 \\
\hline & Gesamt & 434 & 100 \\
\hline \multicolumn{2}{|c|}{ Nicht ausgewählte Fälle } & 0 & 0 \\
\hline \multicolumn{2}{|c|}{ Gesamt } & 434 & 100 \\
\hline
\end{tabular}

Tabelle 16: Zugang und Abstinenzrate - Codierung der abhängigen Variablen der logistischen Regression

\begin{tabular}{|c|c|c|}
\hline \multicolumn{3}{|c|}{ Codierung abhängiger Variablen } \\
\hline Ursprünglicher & & Interner Wert \\
\hline \multirow{2}{*}{ Abstinenzrate } & rückfällig & 0 \\
\hline & abstinent & 1 \\
\hline
\end{tabular}

Tabelle 17: Zugang und Abstinenzrate - Modellzusammenfassung der logistischen Regression; „Schritt 1" deutet darauf hin, dass die logistische Regression durchgeführt wurde. Nagelkerkes R-Quadrat besagt hier, dass durch die im Modell zusammengefassten Variablen 23,9\% der gemeinsamen Varianz aufgeklärt werden.

\begin{tabular}{|l|l|}
\hline Modellzusammenfassung \\
\hline Schritt & $\begin{array}{l}\text { Nagelkerkes } \\
\text { R-Quadrat }\end{array}$ \\
\hline 1 & 0,239 \\
\hline
\end{tabular}


Tabelle 18: Zugang und Abstinenzrate - Klassifizierungstabelle der logistischen Regression; „a“: der Trennwert lautet 0,500; die Tabelle gibt die Übereinstimmung zwischen der aus dem Modell vorhergesagten Abstinenzrate und der beobachteten Abstinenzrate wieder

\begin{tabular}{|c|c|c|c|}
\hline \multicolumn{4}{|l|}{ Klassifizierungstabelle $^{\mathrm{a}}$} \\
\hline \multirow{3}{*}{ Beobachtet } & \multicolumn{3}{|c|}{ Vorhergesagt } \\
\hline & \multicolumn{2}{|c|}{ Abstinenzrate } & \multirow{2}{*}{$\begin{array}{l}\text { Prozentsatz } \\
\text { der Richtiger }\end{array}$} \\
\hline & rückfällig & abstinent & \\
\hline \multirow{2}{*}{ Abstinenzrate } & 133 & 26 & 83,6 \\
\hline & 49 & 57 & 53,8 \\
\hline Gesamtprozentsatz & & & 71,7 \\
\hline
\end{tabular}


Tabelle 19: Zugang und Abstinenzrate - Variablen in der Gleichung nach durchgeführter logistischer Regression

\section{Legende:}

- $\quad$ "a“ = „Schritt 1“ gibt an, dass die logistische Regression durchgeführt wurde

- "RegressionskoeffizientB“ = gibt die Richtung des Effekts auf die abhängige Variable bei Erhöhung des Werts der unabhängigen Variablen um „1“ an

- "Wald“ = statistischer Test für die multiple Regressionsanalyse, der aus dem Verhältnis "Regressionskoeffizient B zum Quadrat / Standardfehler zum Quadrat" das Signifikanzniveau (= "Sig.") errechnet

- $\quad$ "df“" = Freiheitsgrade

- $\quad{ } \operatorname{Exp}(B))^{“}=$ gibt die Änderung des Chancenverhältnisses an bei Erhöhung der unabhängigen Variablen, wobei Werte $>1$ eine Erhöhung des Effekts bedeuten, Werte $<1$ eine Verminderung

- Grau hinterlegt sind die Variablen der Hypothese 1, darauf folgen die Variablen der Hypothese 2

- Als Dummy-Variablen gingen in die Berechnung ein: „Auflage“ („Auflage“ erhielt den Wert „1“), "Geschlecht“ („weiblich“ = „1“), „Wiederaufnahme“ (,Ja“ = „1“), „Zuvor suchtbezogene Hilfe (erhalten)“ (,Ja“ = „1“)

- In Dummy-Variablen umgewandelte kategoriale Variablen sind: „Motivationslage“ (Referenzkategorie (=RK) „Eigenmotiviert“), „Dauer_Arbeitslosigkeit“ (RK „Arbeitslosigkeit < 1 Monat"), „Erwerbssituation_Anfang“ (RK „erwerbstätig“), „Schulabschluss“ (RK „Abitur/(Fach)Hochschulreife“), „Kostenträger“ (RK „Bundesversicherungsanstalt für Angestellte"), „Familienstand“ (RK „verheiratet, zusammenlebend“)

- Alle anderen Variablen sind metrischer Natur

- $\quad$ „IQ“ = Intelligenzquotient, abgeschätzt anhand des Wortschatztests

- "c.I.-Skala“ = Skala zur Erfassung cerebraler Insuffizienzen

- $\quad$ "PSSI“ = Persönlichkeits-Stil-und-Störungs-Inventar

- "LVA“ = Landesversicherungsanstalt, der frühere Name der regionalen Rentenversicherungen

\begin{tabular}{|c|c|c|c|c|c|c|c|c|}
\hline \multicolumn{9}{|c|}{ Variablen in der Gleichung } \\
\hline & & \multirow{2}{*}{$\begin{array}{l}\text { Regressions } \\
\text { koeffizientB }\end{array}$} & \multirow{2}{*}{$\begin{array}{l}\text { Standard- } \\
\text { fehler }\end{array}$} & \multirow{2}{*}{ Wald } & \multirow[t]{2}{*}{ dfSig. } & \multirow[t]{2}{*}{$\operatorname{Exp}(B)$} & \multicolumn{2}{|c|}{\begin{tabular}{|lr}
95 & $\%$ \\
Konfidenzintervall \\
für $\operatorname{EXP}(\mathrm{B})$
\end{tabular}} \\
\hline & & & & & & & $\begin{array}{l}\text { Unterer } \\
\text { Wert }\end{array}$ & $\begin{array}{l}\text { Oberer } \\
\text { Wert }\end{array}$ \\
\hline \multirow{4}{*}{$\begin{array}{l}\text { Schritt } \\
1^{a^{a}}\end{array}$} & Auflage = "Auflage" & $-0,531$ & 0,418 & 1,616 & 10,204 & 40,588 & 0,259 & 1,334 \\
\hline & Motivationslage & & & 7,818 & 20,02 & & & \\
\hline & Ambivalenz-Motivation & $-1,402$ & 0,503 & 7,76 & 10,005 & 0,246 & 0,092 & 0,66 \\
\hline & Fremd-Motivation & $-0,183$ & 0,511 & 0,129 & 10,72 & 0,833 & 0,306 & 2,266 \\
\hline
\end{tabular}


Fortsetzung Tabelle 19: Zugang und Abstinenzrate - Variablen in der Gleichung nach durchgeführter logistischer Regression

\begin{tabular}{|c|c|c|c|c|c|c|c|}
\hline \multicolumn{8}{|l|}{ Variablen in der Gleichung } \\
\hline & \multirow{2}{*}{$\begin{array}{l}\text { Regressions- } \\
\text { koeffizientB }\end{array}$} & \multirow{2}{*}{$\begin{array}{l}\text { Standard- } \\
\text { fehler }\end{array}$} & \multirow{2}{*}{ Wald df } & \multirow[t]{2}{*}{ Sig. } & \multirow[t]{2}{*}{$\operatorname{Exp}(B)$} & \multicolumn{2}{|c|}{\begin{tabular}{|lr}
95 & $\%$ \\
Konfidenzintervall \\
für $\operatorname{EXP}(\mathrm{B})$
\end{tabular}} \\
\hline & & & & & & $\begin{array}{l}\text { Unterer } \\
\text { Wert }\end{array}$ & $\begin{array}{l}\text { Oberer } \\
\text { Wert }\end{array}$ \\
\hline Alter bei Aufnahme & 0,035 & 0,025 & 1,9111 & 0,167 & 1,035 & 0,986 & 1,087 \\
\hline Wartezeit & 0 & 0,003 & 0,0311 & 0,861 & 1 & 0,995 & 1,006 \\
\hline Geschlecht = "weiblich" & $-0,491$ & 0,45 & 1,1911 & 0,275 & 0,612 & 0,253 & 1,478 \\
\hline Wortschatz IQ & 0,003 & 0,018 & 0,0191 & 0,889 & 1,003 & 0,967 & 1,039 \\
\hline Verbalgedächtnis & 0,036 & 0,078 & 0,2161 & 0,642 & 1,037 & 0,89 & 1,208 \\
\hline cl-Skala & 0,005 & 0,023 & 0,0531 & 0,818 & 1,005 & 0,96 & 1,053 \\
\hline $\begin{array}{l}\text { PSSI } \\
\text { (Selbstunsicherheit) }\end{array}$ & 0,015 & 0,024 & 0,3691 & 0,544 & 1,015 & 0,968 & 1,064 \\
\hline $\begin{array}{ll}\text { PSSI } & \text { (spontan- } \\
\text { borderline) }\end{array}$ & $-0,026$ & 0,027 & 0,9481 & 0,33 & 0,975 & 0,925 & 1,027 \\
\hline Abhängigkeitsdauer & $-0,014$ & 0,023 & 0,3911 & 0,532 & 0,986 & 0,942 & 1,031 \\
\hline Dauer_Arbeitslosigkeit & & & 0,5682 & 0,753 & & & \\
\hline $\begin{array}{l}\text { Dauer_Arbeitslosigkeit: } 1 \\
\text { bis } 12 \text { Monate }\end{array}$ & $-0,096$ & 0,551 & $0,03 \quad 1$ & 0,861 & 0,908 & 0,309 & 2,673 \\
\hline $\begin{array}{l}\text { Dauer_Arbeitslosigkeit: > } \\
12 \text { Monate }\end{array}$ & 0,223 & 0,588 & 0,1431 & 0,705 & 1,249 & 0,395 & 3,955 \\
\hline Erwerbssituation_Anfang & & & 0,9463 & 0,814 & & & \\
\hline \begin{tabular}{|l|} 
Erwerbssituation: \\
erwerbslos
\end{tabular} & $-0,389$ & 0,533 & 0,5321 & 0,466 & 0,678 & 0,238 & 1,927 \\
\hline $\begin{array}{l}\text { Erwerbssituation: } \\
\text { berentet }\end{array}$ & 0,357 & 0,783 & 0,2081 & 0,649 & 1,429 & 0,308 & 6,636 \\
\hline $\begin{array}{l}\text { Erwerbssituation: } \\
\text { Sonstiges }\end{array}$ & $-0,166$ & 1,175 & $0,02 \quad 1$ & 0,888 & 0,847 & 0,085 & 8,473 \\
\hline Schulabschluss & & & 3,2566 & 0,776 & & & \\
\hline Schule: keine Angaben & 37,453 & $1,42 E+08$ & & 1 & $1,84 \mathrm{E}+16$ & 0 & . \\
\hline $\begin{array}{ll}\text { Schule: } & \text { ohne } \\
\text { Schulabschluss } & \end{array}$ & $-1,658$ & 1,316 & $1,587 \mid 1$ & 0,208 & 0,191 & 0,014 & 2,513 \\
\hline $\begin{array}{l}\text { Schule: } \\
\text { Sonderschulabschluss }\end{array}$ & 0,239 & 1,311 & 0,0331 & 0,856 & 1,27 & 0,097 & 16,575 \\
\hline $\begin{array}{l}\text { Schule: } \\
\text { Hauptschulabschluss }\end{array}$ & 0,434 & 0,637 & 0,4651 & 0,495 & 1,544 & 0,443 & 5,379 \\
\hline \begin{tabular}{|l|} 
Schule: \\
Realschule/Polytechnisc \\
he Oberschule \\
\end{tabular} & 0,136 & 0,523 & $0,067 \mid 1$ & 0,795 & 1,145 & 0,411 & 3,195 \\
\hline \begin{tabular}{|ll} 
Schule: & Anderer \\
Schulabschluss & \\
\end{tabular} & $-0,408$ & 1,56 & 0,0681 & 0,794 & 0,665 & 0,031 & 14,149 \\
\hline Kostenträger & & & 2,7176 & 0,843 & & & \\
\hline Kostenträger: Andere & 0,793 & 1,166 & 0,4631 & 0,496 & 2,21 & 0,225 & 21,716 \\
\hline $\begin{array}{ll}\text { Kostenträger: Andere } \\
\text { LVAen }\end{array}$ & $-0,208$ & 0,957 & $0,047 \mid 1$ & 0,828 & 0,812 & 0,124 & 5,304 \\
\hline $\begin{array}{l}\text { Kostenträger: } \\
\text { Krankenkassen }\end{array}$ & $-0,364$ & 0,825 & 0,1951 & 0,659 & 0,695 & 0,138 & 3,502 \\
\hline $\begin{array}{l}\text { Kostenträger: } \\
\text { Bahn/Knappschaft /See }\end{array}$ & $-0,765$ & 0,601 & 1,6231 & 0,203 & 0,465 & 0,143 & 1,51 \\
\hline
\end{tabular}


Fortsetzung Tabelle 19: Zugang und Abstinenzrate - Variablen in der Gleichung nach durchgeführter logistischer Regression

\begin{tabular}{|l|l|l|l|l|l|l|l|l|}
\hline Variablen in der Gleichung & \multicolumn{5}{|c|}{\begin{tabular}{l}
95 \\
Konfidenzintervall \\
\hline
\end{tabular}} \\
\hline
\end{tabular}




\section{Zugang und Erwerbssituation nach einem Jahr}

Tabelle 20: Zugang und Erwerbssituation nach einem Jahr - Zusammenfassung der Fallverarbeitung der logistischen Regression

\begin{tabular}{|c|c|c|c|}
\hline \multicolumn{4}{|c|}{ Zusammenfassung der Fallverarbeitung } \\
\hline & & $\mathrm{N}$ & Prozent \\
\hline \multirow{3}{*}{$\begin{array}{l}\text { Ausgewählte } \\
\text { Fälle }\end{array}$} & $\begin{array}{l}\text { Einbezogen in } \\
\text { Analyse }\end{array}$ & 127 & 29,3 \\
\hline & \begin{tabular}{|l} 
Fehlende \\
Fälle \\
\end{tabular} & 307 & 70,7 \\
\hline & Gesamt & 434 & 100 \\
\hline \multicolumn{2}{|c|}{ Nicht ausgewählte Fälle } & 0 & 0 \\
\hline \multicolumn{2}{|l|}{ Gesamt } & 434 & 100 \\
\hline
\end{tabular}

Tabelle 21: Zugang und Erwerbssituation nach einem Jahr -

Codierung der abhängigen Variablen der logistischen Regression

Codierung abhängiger Variablen

Ursprünglicher Wert $\quad$ Interner Wert

\begin{tabular}{l|l|l|}
\hline Arbeit & Kaine Arbeit & 0 \\
\cline { 2 - 3 } Ja Nein & Arbeit & 1 \\
\hline
\end{tabular}

Tabelle 22: Zugang und Erwerbssituation nach einem Jahr -

Modellzusammenfassung der logistischen Regression; „Schritt 1“ meint, dass die logistische Regression durchgeführt wurde. Nagelkerkes R-Quadrat besagt hier, dass durch die im Modell zusammengefassten Variablen 81,7 \% der gemeinsamen Varianz aufgeklärt werden.

\begin{tabular}{|l|l|}
\hline Modellzusammenfassung \\
\hline Schritt & $\begin{array}{l}\text { Nagelkerkes } \\
\text { R-Quadrat }\end{array}$ \\
\hline 1 & 0,817 \\
\hline
\end{tabular}


Tabelle 23: Zugang und Erwerbssituation nach einem Jahr -

Klassifizierungstabelle der logistischen Regression; „a“: der Trennwert lautet 0,500; die Tabelle gibt die Übereinstimmung zwischen dem aus dem Modell vorhergesagten Anteil an Erwerbstätigkeit und dem beobachteten Anteil an Erwerbstätigkeit nach einem Jahr wieder

\begin{tabular}{|l|l|l|l|l|l|}
\hline Klassifizierungstabelle $^{\mathbf{a}}$ & \multicolumn{2}{l|}{ Vorhergesagt } \\
\cline { 4 - 6 } & \multirow{3}{*}{ Beobachtet } & \multicolumn{2}{|l|}{ Arbeit Ja/Nein } & $\begin{array}{l}\text { Prozentsatz } \\
\text { der } \\
\text { Richtigen }\end{array}$ \\
\cline { 3 - 6 } & & 0 & 1 & 94,4 \\
\hline \multirow{3}{*}{ Schritt 1} & Arbeit & 0 & 68 & 4 & 98,2 \\
\cline { 3 - 5 } & Ja/Nein & 1 & 1 & 54 & 96,1 \\
\cline { 2 - 4 } & Gesamtprozentsatz & & & \\
\hline
\end{tabular}


Tabelle 24: Zugang und Erwerbssituation nach einem Jahr -

Variablen in der Gleichung nach durchgeführter logistischer Regression

Legende:

- " „a“ = „Schritt 1“ gibt an, dass die logistische Regression durchgeführt wurde

- "RegressionskoeffizientB“ = gibt die Richtung des Effekts auf die abhängige Variable bei Erhöhung des Werts der unabhängigen Variablen um "1" an

- „Wald“ = statistischer Test für die multiple Regressionsanalyse, der aus dem Verhältnis "Regressionskoeffizient B zum Quadrat / Standardfehler zum Quadrat" das Signifikanzniveau (= "Sig.") errechnet

- $\quad$ "df" = Freiheitsgrade

- ${ } \operatorname{Exp}(B))^{“}=$ gibt die Änderung des Chancenverhältnisses an bei Erhöhung der unabhängigen Variablen, wobei Werte $>1$ eine Erhöhung des Effekts bedeuten, Werte $<1$ eine Verminderung

- Grau hinterlegt sind die Variablen der Hypothese 1, darauf folgen die Variablen der Hypothese 2

- Als Dummy-Variablen gingen in die Berechnung ein: „Auflage“ („Auflage“ erhielt den Wert „1“), „Geschlecht“ (,weiblich“ = „1“), „Wiederaufnahme“ (,Ja“ = „1“), „Zuvor suchtbezogene Hilfe (erhalten)“ („Ja“ = „1“)

- In Dummy-Variablen umgewandelte kategoriale Variablen sind: „Motivationslage“ (Referenzkategorie (=RK) „Eigenmotiviert“), „Dauer_Arbeitslosigkeit“ (RK „Arbeitslosigkeit < 1 Monat“), „Erwerbssituation_Anfang“ (RK „erwerbstätig“), „Schulabschluss“ (RK „Abitur/(Fach)Hochschulreife“), „Kostenträger“ (RK „Bundesversicherungsanstalt für Angestellte"), „Familienstand“ (RK „verheiratet, zusammenlebend“)

- Alle anderen Variablen sind metrischer Natur

- „IQ“ = Intelligenzquotient, abgeschätzt anhand des Wortschatztests

- "c.I.-Skala“ = Skala zur Erfassung cerebraler Insuffizienzen

- $\quad$ "PSSI“ = Persönlichkeits-Stil-und-Störungs-Inventar

- "LVA“ = Landesversicherungsanstalt, der frühere Name der regionalen Rentenversicherungen

\begin{tabular}{|c|c|c|c|c|c|c|c|c|c|}
\hline \multicolumn{10}{|c|}{ Variablen in der Gleichung } \\
\hline & & \multirow[b]{2}{*}{$\begin{array}{l}\text { Regressions- } \\
\text { koeffizientB }\end{array}$} & \multirow[b]{2}{*}{$\begin{array}{l}\text { Standard } \\
\text {-fehler }\end{array}$} & \multirow[b]{2}{*}{ Wald } & \multirow[b]{2}{*}{$\begin{array}{l}d \\
f\end{array} s$} & \multirow[b]{2}{*}{ Sig. } & \multirow[b]{2}{*}{$\operatorname{Exp}(B)$} & \multicolumn{2}{|c|}{$\begin{array}{l}95 \% \text { Konfidenzintervall } \\
\text { für } \operatorname{EXP}(B)\end{array}$} \\
\hline & & & & & & & & $\begin{array}{l}\text { Unterer } \\
\text { Wert }\end{array}$ & $\begin{array}{l}\text { Oberer } \\
\text { Wert }\end{array}$ \\
\hline \multirow{4}{*}{$\begin{array}{l}\text { Schri } \\
\text { tt } 1^{a}\end{array}$} & \begin{tabular}{|l} 
Auflage \\
"Auflage"
\end{tabular} & $=0,085$ & 1,492 & 0,003 & & 0,954 & 1,089 & 0,058 & 20,287 \\
\hline & Motivationslage & & & 4,822 & 2 & 0,09 & & & \\
\hline & $\begin{array}{l}\text { Ambivalenz- } \\
\text { Motivation }\end{array}$ & $-4,473$ & 2,057 & 4,728 & & 0,03 & 0,011 & 0 & 0,643 \\
\hline & Fremd-Motivation & 0,158 & 1,618 & 0,01 & & 0,922 & 1,171 & 0,049 & 27,901 \\
\hline
\end{tabular}


Fortsetzung: Tabelle 24: Zugang und Erwerbssituation nach einem Jahr Variablen in der Gleichung nach durchgeführter logistischer Regression

\begin{tabular}{|c|c|c|c|c|c|c|c|}
\hline \multicolumn{8}{|c|}{ Variablen in der Gleichung } \\
\hline & \multirow[b]{2}{*}{$\begin{array}{l}\text { Regressions- } \\
\text { koeffizientB }\end{array}$} & \multirow[b]{2}{*}{$\begin{array}{l}\text { Standard } \\
\text {-fehler }\end{array}$} & \multirow[b]{2}{*}{ Wald $\left.\right|_{f} ^{d}$} & \multirow[b]{2}{*}{ Sig. } & \multirow[b]{2}{*}{$\operatorname{Exp}(B)$} & \multicolumn{2}{|c|}{$\begin{array}{l}95 \% \text { Konfidenzintervall } \\
\text { für } \operatorname{EXP}(B)\end{array}$} \\
\hline & & & & & & $\begin{array}{l}\text { Unterer } \\
\text { Wert }\end{array}$ & $\begin{array}{l}\text { Oberer } \\
\text { Wert }\end{array}$ \\
\hline $\begin{array}{l}\text { Alter } \\
\text { Aufnahme }\end{array}$ & $\mathrm{i} 0,028$ & 0,09 & 0,0971 & 0,756 & 1,028 & 0,862 & 1,227 \\
\hline Wartezeit & $-0,036$ & 0,013 & 7,4751 & 0,006 & 0,965 & 0,94 & 0,99 \\
\hline \begin{tabular}{|l|} 
Geschlecht \\
"weiblich" \\
\end{tabular} & $=1,094$ & 2,091 & 0,2741 & 0,601 & 2,987 & 0,05 & 179,905 \\
\hline Wortschatz IQ & 0,238 & 0,097 & 6,0751 & 0,014 & 1,269 & 1,05 & 1,533 \\
\hline Verbalgedächtnis & 1,033 & 0,392 & 6,9581 & 0,008 & 2,809 & 1,304 & 6,052 \\
\hline cl-Skala & $-0,107$ & 0,063 & 2,8411 & 10,092 & 0,899 & 0,794 & 1,018 \\
\hline \begin{tabular}{|l|} 
PSSI \\
(Selbstunsicherhe \\
it)
\end{tabular} & $-0,081$ & 0,075 & \begin{tabular}{|l|l|}
1,173 & 1
\end{tabular} & $\mid 0,279$ & 0,922 & 0,796 & 1,068 \\
\hline \begin{tabular}{|l} 
PSSI (spontan- \\
borderline)
\end{tabular} & 0,086 & 0,079 & \begin{tabular}{|l|l|}
1,172 & 1
\end{tabular} & $\mid 0,279$ & 1,09 & 0,933 & 1,274 \\
\hline $\begin{array}{l}\text { Abhängigkeitsdau } \\
\text { er }\end{array}$ & $-0,404$ & 0,154 & 6,88 & 10,009 & 0,668 & 0,494 & 0,903 \\
\hline $\begin{array}{l}\text { Dauer_Arbeitslosi } \\
\text { gkeit }\end{array}$ & & & 9,54 & 20,008 & & & \\
\hline $\begin{array}{l}\text { Dauer_Arbeitslosi } \\
\text { gkeit: } 1 \text { bis } 12 \\
\text { Monate }\end{array}$ & $-16,352$ & 5,382 & 9,2311 & $\mid 0,002$ & 0 & 0 & 0,003 \\
\hline $\begin{array}{|lr|}\text { Dauer_Arbeitslosi } \\
\text { gkeit: } & > \\
\text { Monate } & \\
\end{array}$ & $-14,371$ & 4,668 & \begin{tabular}{|l|l|}
9,478 & 1
\end{tabular} & 10,002 & 0 & 0 & 0,005 \\
\hline \begin{tabular}{|l|} 
Erwerbssituation \\
Anfang
\end{tabular} & & & 4,3773 & 30,224 & & & \\
\hline \begin{tabular}{|l|} 
Erwerbssituation: \\
erwerbslos
\end{tabular} & 5,642 & 2,697 & 4,3771 & 0,036 & $\begin{array}{l}281,91 \\
7\end{array}$ & 1,428 & 55646,76 \\
\hline \begin{tabular}{|l|} 
Erwerbssituation: \\
berentet
\end{tabular} & $-73,328$ & $2,84 \mathrm{E}+08$ & 0 & 1 & 0 & 0 & \\
\hline \begin{tabular}{|l|} 
Erwerbssituation: \\
Sonstiges
\end{tabular} & 0,973 & 70,836 & 0 & 0,989 & 2,647 & 0 & $5,23 E+60$ \\
\hline Schulabschluss & & & 6,17 & 0,404 & & & \\
\hline $\begin{array}{|ll|}\text { Schule: keine } \\
\text { Angaben }\end{array}$ & 3,135 & $9,14 \mathrm{E}+08$ & 0 & 11 & 22,994 & 0 & \\
\hline $\begin{array}{|ll|}\text { Schule: } & \text { ohne } \\
\text { Schulabschluss }\end{array}$ & $-41,97$ & $8,85 E+08$ & & 1 & 0 & 0 & \\
\hline $\begin{array}{l}\text { Schule: } \\
\text { Sonderschulabsc } \\
\text { hluss }\end{array}$ & 11,341 & 5,172 & 4,8081 & 0,028 & $\begin{array}{l}84221 \\
872\end{array}$ & 3,333 & $2,13 \mathrm{E}+09$ \\
\hline $\begin{array}{l}\text { Schule: } \\
\text { Hauptschulabschl } \\
\text { uss }\end{array}$ & 0,573 & 2,431 & \begin{tabular}{|l|l|}
0,056 & 1
\end{tabular} & 0,814 & 1,773 & 0,015 & 208,188 \\
\hline $\begin{array}{l}\text { Schule: } \\
\text { Realschule/Polyte } \\
\text { chnische } \\
\text { Oberschule } \\
\end{array}$ & 1,598 & 2,155 & 0,55 & $1 \mid 0,458$ & 4,944 & 0,072 & 337,322 \\
\hline \begin{tabular}{|l|} 
Schule: Anderer \\
Schulabschluss
\end{tabular} & 49,504 & $1,42 \mathrm{E}+08$ & 0 & 1 & $\begin{array}{l}3,16 \mathrm{E}+ \\
21\end{array}$ & 0 & \\
\hline
\end{tabular}


Fortsetzung: Tabelle 24: Zugang und Erwerbssituation nach einem Jahr Variablen in der Gleichung nach durchgeführter logistischer Regression

\begin{tabular}{|c|c|c|c|c|c|c|c|}
\hline \multicolumn{8}{|c|}{ Variablen in der Gleichung } \\
\hline & \multirow[b]{2}{*}{$\begin{array}{l}\text { Regressions- } \\
\text { koeffizientB }\end{array}$} & \multirow[b]{2}{*}{$\begin{array}{l}\text { Standard } \\
\text {-fehler }\end{array}$} & \multirow[b]{2}{*}{ Wald } & \multirow[b]{2}{*}{ Sig. } & \multirow[b]{2}{*}{$\operatorname{Exp}(B)$} & \multicolumn{2}{|c|}{$\begin{array}{l}95 \% \text { Konfidenzintervall } \\
\text { für } \operatorname{EXP}(B)\end{array}$} \\
\hline & & & & & & $\begin{array}{l}\text { Unterer } \\
\text { Wert }\end{array}$ & $\begin{array}{l}\text { Oberer } \\
\text { Wert }\end{array}$ \\
\hline Kostenträger & & & 9,1986 & 60,163 & & & \\
\hline $\begin{array}{l}\text { Kostenträger: } \\
\text { Andere }\end{array}$ & $-4,168$ & 4,519 & $0,851 \mid 1$ & 10,356 & 0,015 & 0 & 108,705 \\
\hline $\begin{array}{l}\text { Kostenträger: } \\
\text { Andere LVAen }\end{array}$ & 1,338 & 4,501 & $0,088 \mid 1$ & 10,766 & 3,81 & 0,001 & 25849,257 \\
\hline $\begin{array}{l}\text { Kostenträger: } \\
\text { Krankenkassen }\end{array}$ & 22,968 & $1,74 \mathrm{E}+08$ & 0 & 11 & $\begin{array}{l}9,44 \mathrm{E}+ \\
09\end{array}$ & 0 & \\
\hline $\begin{array}{l}\text { Kostenträger: } \\
\text { Bahn/Knappschaft/ } \\
\text { See }\end{array}$ & $-3,749$ & 2,396 & $2,449 \mid 1$ & 10,118 & 0,024 & 0 & 2,576 \\
\hline $\begin{array}{l}\text { Kostenträger: LVA } \\
\text { Sachsen-Anhalt }\end{array}$ & $-0,007$ & 1,705 & 0 & 10,997 & 0,993 & 0,035 & 28,052 \\
\hline $\begin{array}{l}\text { Kostenträger: LVA } \\
\text { Thüringen }\end{array}$ & 13,402 & 4,68 & 8,199 & 10,004 & $\begin{array}{l}661151 \\
, 35\end{array}$ & 68,596 & $6,37 \mathrm{E}+09$ \\
\hline Familienstand & & & 8,366 & 40,079 & & & \\
\hline \begin{tabular}{|l|} 
Familienstand: \\
verheiratet \\
getrennt lebend
\end{tabular} & 5,215 & 3,386 & \begin{tabular}{|l|l|}
2,372 & 1 \\
\end{tabular} & 10,124 & $\begin{array}{l}183,95 \\
2\end{array}$ & 0,241 & 140199,147 \\
\hline Familienstand: ledig & 4,773 & 2,171 & $4,835 \mid 1$ & 10,028 & $\begin{array}{l}118,32 \\
6\end{array}$ & 1,68 & 8334,35 \\
\hline \begin{tabular}{|l} 
Familienstand: \\
geschieden
\end{tabular} & 6,689 & 2,314 & $8,353 \mid 1$ & 10,004 & $\begin{array}{l}803,13 \\
9\end{array}$ & 8,609 & 74926,545 \\
\hline $\begin{array}{l}\text { Familienstand: } \\
\text { verwitwet }\end{array}$ & $-30,809$ & $8,85 \mathrm{E}+08$ & & \begin{tabular}{|l|l|}
1 & 1 \\
\end{tabular} & 0 & 0 & \\
\hline $\begin{array}{l}\text { Wiederaufnahme = } \\
\text { "Ja" (Dummy) }\end{array}$ & $=-0,319$ & 1,486 & 0,046 & 10,83 & 0,727 & 0,039 & 13,377 \\
\hline $\begin{array}{l}\text { Zuvor } \\
\text { suchtbezogene } \\
\text { Hilfe = "Ja" } \\
\text { (Dummy) } \\
\end{array}$ & 1,605 & 2,324 & $0,477 \mid 1$ & 10,49 & 4,978 & 0,052 & 473,016 \\
\hline \begin{tabular}{|l|} 
Körperbezogene \\
Komorbidität
\end{tabular} & 1,154 & 1,195 & $0,933 \mid 1$ & 10,334 & 3,171 & 0,305 & 32,968 \\
\hline \begin{tabular}{|l} 
Psychische \\
Erkrankungen
\end{tabular} & 3,732 & 1,897 & 3,8691 & 10,049 & 41,757 & 1,013 & 1720,556 \\
\hline $\begin{array}{l}\text { Alkoholbezogene } \\
\text { Erkrankungen }\end{array}$ & $-1,69$ & 3,076 & $0,302 \mid 1$ & 10,583 & 0,184 & 0 & 76,617 \\
\hline $\begin{array}{l}\text { Zweite } \\
\text { Suchtdiagnose } \\
\text { (ohne Tabak- } \\
\text { abhängigkeit) } \\
\end{array}$ & $-2,215$ & 3,32 & $0,445 \mid 1$ & 10,505 & 0,109 & 0 & 73,147 \\
\hline Tabak-abhängigkeit & 4,227 & 2,215 & $3,643 \mid 1$ & 10,056 & 68,545 & 0,893 & 5263,912 \\
\hline $\begin{array}{l}\text { Persönlichkeits- } \\
\text { störung }\end{array}$ & 7,319 & 3,197 & 5,242 & 10,022 & $\begin{array}{l}1509,4 \\
38\end{array}$ & 2,869 & 794233,729 \\
\hline \begin{tabular}{|l} 
Intelligenz- \\
minderung
\end{tabular} & $-38,166$ & $3,77 \mathrm{E}+08$ & 0 & \begin{tabular}{|l|l|}
1 & 1 \\
\end{tabular} & 0 & 0 & \\
\hline Konstante & $-29,21$ & 13,082 & 4,986 & 10,026 & 0 & & \\
\hline
\end{tabular}




\section{Danksagung}

Der Verfasser spricht folgenden Personen, die an dieser Arbeit mitgewirkt haben, seinen Dank aus:

- Prof. Dr. med. Peter Brieger, ärztlicher Direktor des Bezirkskrankenhauses Kempten: Mentor der Arbeit

- Dietmar Kemmann, leitender Psychologe des Diakonie-Krankenhauses Elbingerode: Unterstützung bei der Datenerhebung und Beratung der statistischen Auswertung

- PD Dr. rer soc Reinhold Kilian, Universität UIm: Beratung und Begleitung bei methodischen Fragen der Arbeit

- Isabelle Lehmann, Chefarztsekretärin der Rehabilitationsklinik Elbingerode: schriftliche Darstellung, Mitwirkung bei Formatierung und Layout 


\section{Lebenslauf}

Eckart Grau, geb. 05.09.1961

Schulbildung:

$1968-1972$

Grundschule Stuttgart-Degerloch

$1972-1981$

Wilhelmsgymnasium Stuttgart-Degerloch

Kirchliche Jugendarbeit:

$1981-1983$

hauptamtlich Jugendarbeit im Evangelischen Jugendwerk Degerloch

Studium:

1983

1985

1987

1990

$1990-1993$

$1993-1994$

1994
Medizinstudium an der Ruprecht-Karls-Universität Heidelberg ärztliche Vorprüfung

1. Abschnitt der ärztlichen Prüfung

2. Abschnitt der ärztlichen Prüfung theologische Studien

praktisches Jahr, Diakonie-Krankenhaus Schwäbisch Hall, Innere Abteilung

3. Abschnitt der ärztlichen Vorprüfung
Ausgeübter Arztberuf: $1994-1996$

$1996-1997$

seit 1998

$04 / 98-12 / 99$

$01 / 00-08 / 01$

seit 09/01

seit 12.03 .04

seit 01.10 .2004

seit 28.08.2006
Arzt im Praktikum, Diakonie-Krankenhaus Schwäbisch Hall, Innere Medizin

Weiterbildungsassistenz für Facharzt

Psychotherapeutische Medizin,

Burg-Klinik Stadtlengsfeld

Weiterbildungsassistenz für Facharzt

Psychotherapeutische Medizin im Diakonie-

Krankenhaus Elbingerode

Bereich Psychiatrie und Rehabilitation Sucht

Psychotherapeutische Medizin

Bereich Psychiatrie und Rehabilitation Sucht

Facharzt für Psychotherapeutische und

Psychosomatische Medizin

Oberarzt Reha/Sucht

Facharzt für Psychotherapeutische und

Psychosomatische Medizin

Sozialmedizin/Suchtmedizin

Chefarzt Reha/Sucht

Elbingerode, 07.07.2011 